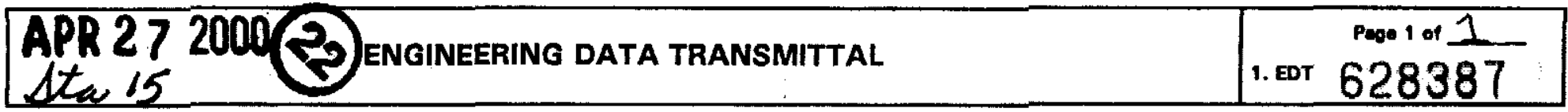

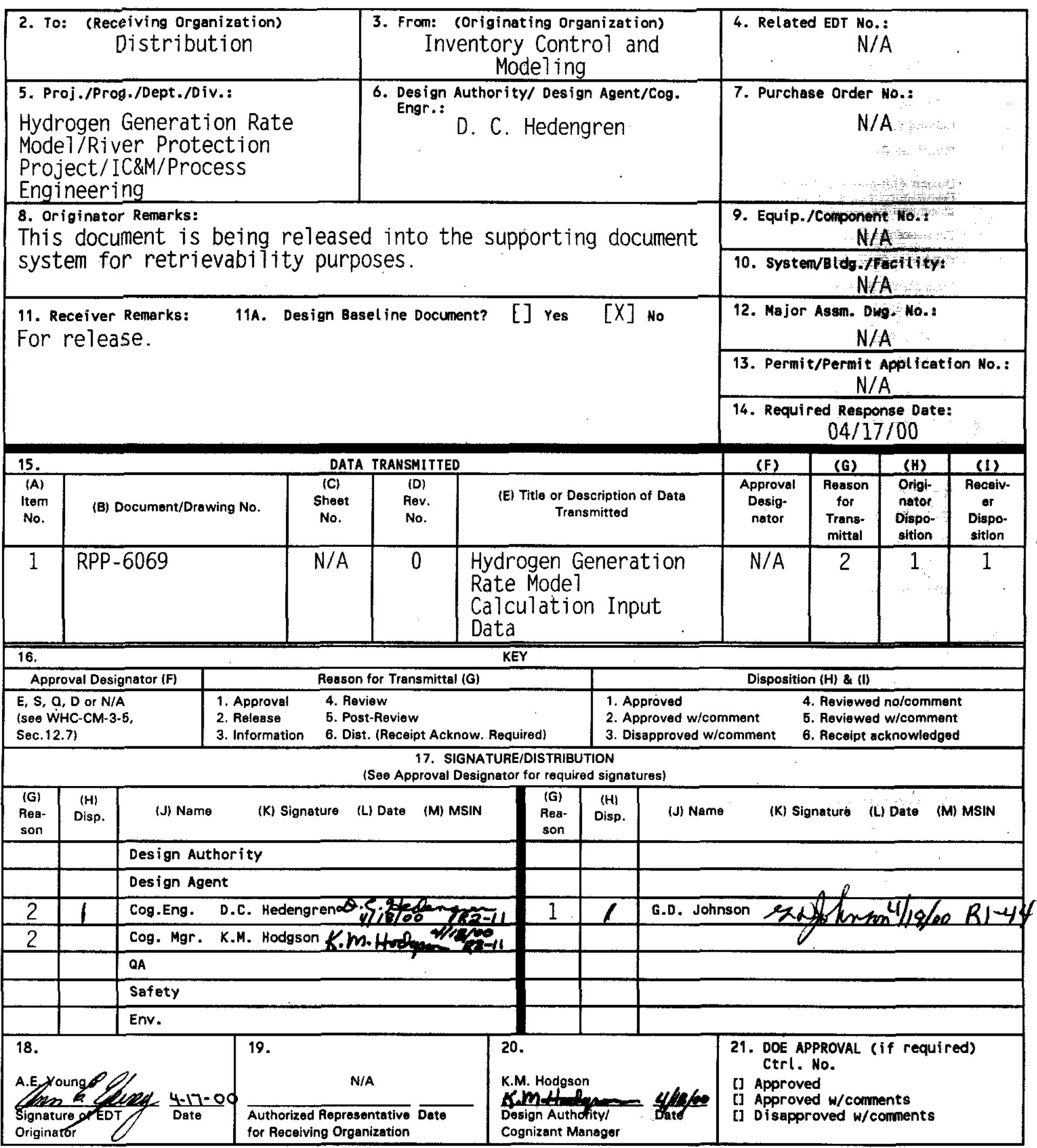

BO-7400-172-2 (05/96) GEF097 


\begin{tabular}{|c|c|c|c|c|c|}
\hline \multicolumn{6}{|c|}{ DISTRIBUTION SHEET } \\
\hline \multirow[b]{2}{*}{ Distribution } & \multirow{2}{*}{\multicolumn{3}{|c|}{$\begin{array}{r}\text { Inventory } \\
\text { Mod }\end{array}$}} & \multicolumn{2}{|l|}{ Page 1 of 1} \\
\hline & & & & \multicolumn{2}{|c|}{ Date $\quad 04 / 17 / 00$} \\
\hline \multicolumn{4}{|l|}{ Project Title/Work Order } & \multicolumn{2}{|c|}{ EDT No. $\quad$ EDT-628387 } \\
\hline \multicolumn{4}{|c|}{$\begin{array}{l}\text { RPP-6069, Rev. 0. "Hydrogen Generation Rate Model Calculation } \\
\text { Input Data" }\end{array}$} & \multicolumn{2}{|l|}{ ECN No. N/A } \\
\hline Name & MSIN & $\begin{array}{c}\text { Text } \\
\text { With } \\
\text { All } \\
\text { Attach }\end{array}$ & Text Only & $\begin{array}{l}\text { Attach. } \\
\text { Appendix } \\
\text { Only }\end{array}$ & $\begin{array}{l}\text { EDT/ECN } \\
\text { Only }\end{array}$ \\
\hline
\end{tabular}

CH2M HILL Hanford Group. Inc.

S. A. Barker

W. B. Barton

D. R. Bratzel

R. J. Cash

D. C. Hedengren

K. M. Hodgson

G. D. Johnson

T.C.S.R.C.

$\begin{array}{ll}\text { R1 }-44 & X \\ \text { R2-11 } & \text { X } \\ \text { R1-44 } & \text { X } \\ \text { R1-44 } & X \\ \text { R2-11 } & X \\ \text { R2-11 } & x \\ \text { R1-44 } & \text { X } \\ \text { R1-10 } & \text { X }\end{array}$

Lockheed Martin Services, Inc.

Central Files

B1-07 $\quad x$

Meier Enterprises. Inc.

S. W. Bisping

M. A. Kufah1

R2-11 $\quad x$

R2-11 $\quad X$

Office of River Protection

DOE Reading Room

H2-53 X 


\section{Hydrogen Generation Rate Model Calculation Input Data}

M. A. Kufahl

Meier Enterprises. Inc.

D. C. Hedengren

CH2M HILL Hanford Group, Inc.. Richland, WA 99352

U.S. Department of Energy Contract DE-AC06-96RL13200

EDT/ECN: EDT-628387 UC: 2070

Org Code: 74B40 CACN/COA: 101898/BA10

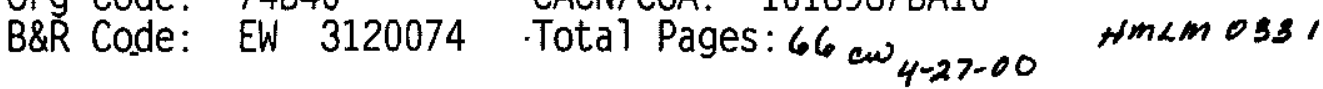

Key Words: Hydrogen Generation Rate. Hydrogen, Generation Rate. Model. Calculation Input Data, Calculation Input. Data

Abstract: N/A

TRADEMARK DISCLAIMER. Reference herein to any specific commercial product, process, or service by trade name, trademark, manufacturer, or otherwise, does not necessarily constitute or imply its endorsement, recommendation, or favoring by the United States Government or any agency thereof or its contractors or subcontractors.

Printed in the United States of America. To obtain copies of this document, contact: Document Control Services, P.0. Box 950, Mailstop H6-08, Richland WA 99352, Phone (509) 372-2420;

Fax (509) 376.4989.

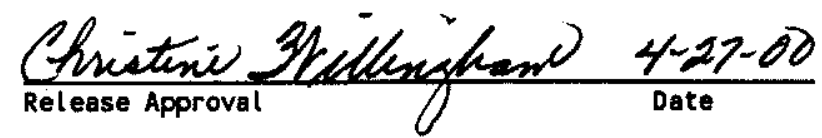

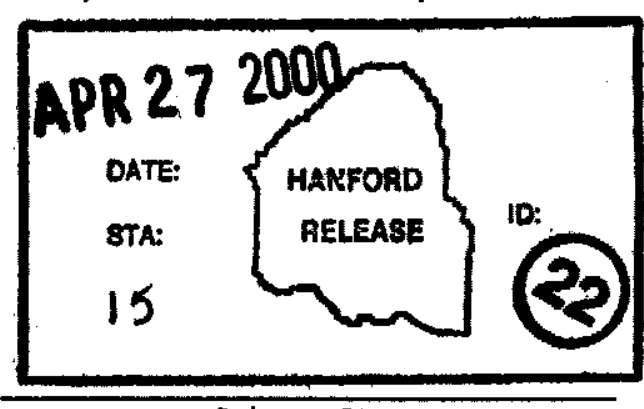

Release Stanp 


\section{Hydrogen Generation Rate Model Calculation Input Data}

M. A. Kufahl

Meier Enterprises, Inc.

D. C. Hedengren

CH2M HILL Hanford Group, Inc.

Date Published

March 2000

Prepared for the U. S. Department of Energy

Office of River Protection

Approved for public release; distribution is unlimited 
RPP - 6069 Rev. 0

This page intentionally left blank 


\section{TABLE OF CONTENTS}

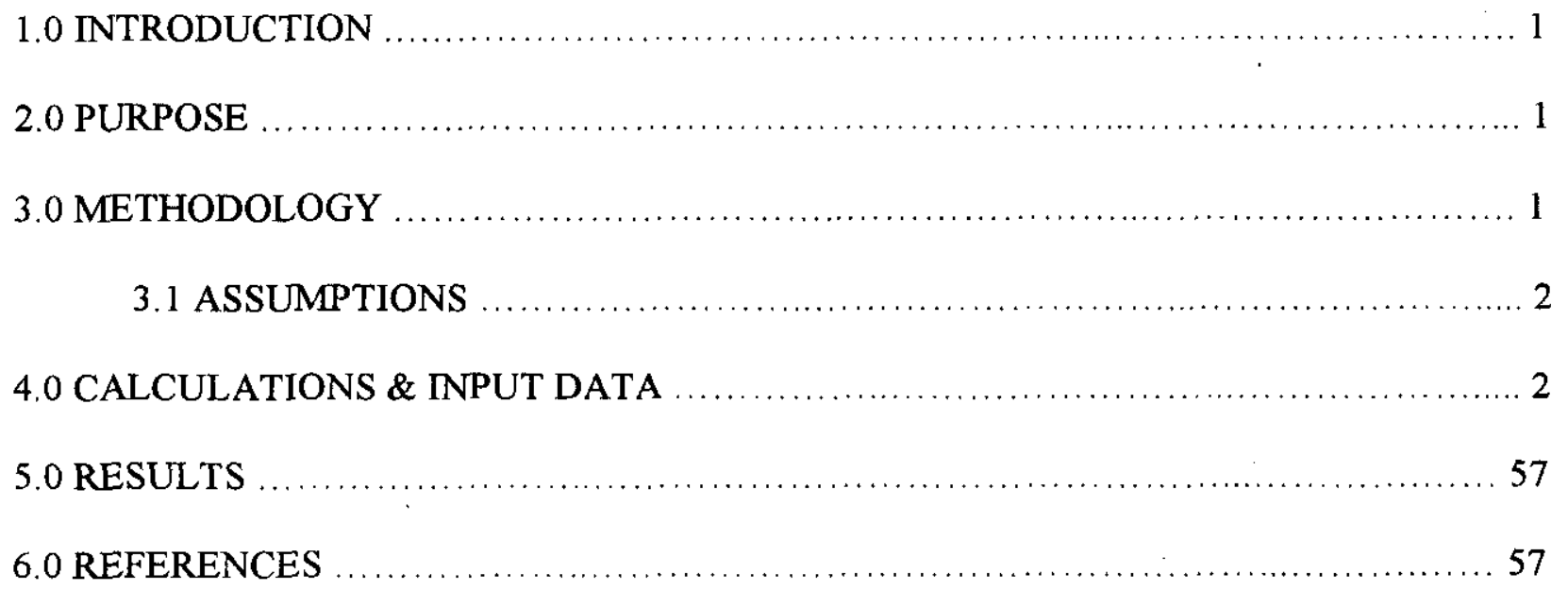




\section{LIST OF TABLES}

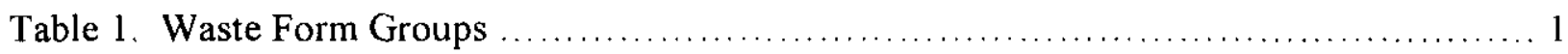

Table 2, Best Basis Calculation Detail Data with Distribution Numbers .................... 5

Table 3. Tank Characterization Database Weight $\% \mathrm{H}_{2} \mathrm{O}$ with Distribution Numbers ......... 27

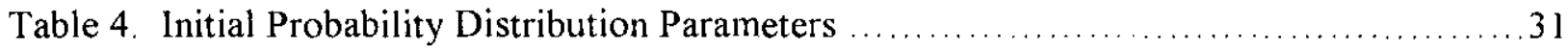

Table 5. Distribution Values Generated by CB Statistical Analysis Software ................. 35

Table 6. Best Basis Calculation Detail Data with Distribution Values and Wt $\% \mathrm{H}_{2} \mathrm{O}$ Data ....39

\section{LIST OF FIGURES}

Figure 1. Data Set for Distribution Analysis, Input Conditions and Distribution Number 29

Figure 2. Frequency Chart Output from Crystal Ball (CB) Statistical Analysis Software ...... 33

Figure 3. Distribution Output of CB Statistical Analysis Software ...................... 33 


\section{LIST OF TERMS}

\begin{tabular}{|c|c|}
\hline $\mathrm{Al}$ & Aluminum \\
\hline $\mathrm{BBCD}$ & Best Basis Calculation Detail \\
\hline BBI & Best Basis Inventory \\
\hline $\mathrm{CB}$ & Crystal Ball $^{\circledR}$ \\
\hline $\mathrm{Cs}^{137}$ & Cesium 137 \\
\hline Conc & Concentration \\
\hline $\mathrm{D}$ & Density \\
\hline $\begin{array}{l}\text { DST } \\
\mathrm{ft}^{3}\end{array}$ & $\begin{array}{l}\text { Double shell tank } \\
\text { cubic feet }\end{array}$ \\
\hline g & gram \\
\hline $\mathrm{H}_{2} \mathrm{O}$ & Water \\
\hline $\mathrm{kL}$ & kiloliters \\
\hline $\mathrm{L}$ & Liquid \\
\hline LIQ & Liquid \\
\hline $\begin{array}{l}\text { MIX-NL } \\
\mathrm{mL}\end{array}$ & $\begin{array}{l}\text { Mixed Sludge-Saltcake without liquid } \\
\text { milliliter }\end{array}$ \\
\hline $\mathrm{NO}_{2}$ & Nitrite \\
\hline $\mathrm{NO}_{3}$ & Nitrate \\
\hline$\%$ & Percent \\
\hline S & Solid \\
\hline $\mathrm{SC}$ & Saltcake \\
\hline SC/SS-LIQ & Saltcake/Salt Slurry with liquid \\
\hline $\mathrm{SC} / \mathrm{SS}-\mathrm{NL}$ & Saltcake/Salt Slurry without liquid \\
\hline SL & Sludge \\
\hline SL-LIQ & Sludge with liquid \\
\hline SL-NL & Sludge without liquid \\
\hline $\mathrm{Sr}^{90}$ & Strontium 90 \\
\hline SST & Single shell tank \\
\hline $\mathrm{T}$ & Total \\
\hline $\mathrm{TCD}$ & Tank Characterization Database \\
\hline TOC & Total Organic Carbon \\
\hline TWINS & Tank Waste Information Network \\
\hline$\mu g$ & microgram \\
\hline$\mu \mathrm{Ci}$ & microcuries \\
\hline V & Volume \\
\hline $\mathrm{Wt} \%$ & Weight percent \\
\hline
\end{tabular}

${ }^{\circledR}$ Crystal Ball is a registered trademark of Decisioneering, Inc., All Rights Reserved 
RPP - 6069 Rev. 0

This page intentionally left blank 


\subsection{Introduction}

This report documents the procedures and techniques utilized in the collection and analysis of analyte input data values in support of the flammable gas hazard safety analyses. This document represents the analyses of data current at the time of its writing and does not account for data available since then.

\subsection{Purpose}

The purpose of this document is to validate the waste tank input data for the hydrogen generation rate model and update the input values with the most current waste tank information. This calculation note also provides value estimates for those tanks missing data and combines the input data values for both Single Shell (SST) and Double Shell (DST) waste tanks.

\subsection{Methodology}

In this calculation note the Best Basis Calculation Detail (BBCD), part of the Best Basis Inventory (BBI) database contained within the Tank Waste Information Network (TWINS3) website, was queried for the desired constituents $\left(\mathrm{Al}, \mathrm{NO}_{2}, \mathrm{NO}_{3}, \mathrm{TOC}, \mathrm{Cs}^{137}, \mathrm{Sr}^{90}\right)$ on a tank by tank basis. The input data collected for the individual waste tanks were categorized into three waste layer types, liquid, salt cake and sludge. The BBCD input data values were collected on 11/23/99 and are current as of this date.

The input data values for the weight percent water $\left(\mathrm{H}_{2} \mathrm{O}\right.$ wt \%) were queried for all tanks from the sample analysis results in the Tank Characterization Database (TCD), also included within the TWINS3 website. The data were filtered to select the primary and duplicate analysis results and then separated into liquid and solid datasets with all questionable values removed for use in averaging calculations. The results for the liquid and solid data sets were then averaged for each tank on a layer basis using Microsoft Excel ${ }^{\circledast}$. The total $\mathrm{H}_{2} \mathrm{O}$ wt \% for each tank was calculated as a mass ratio using the waste layer densities and volumes.

The tanks were defined by layer volumes and grouped according to waste form subsets, Barker et al. (1999), and described in Table 1.

\begin{tabular}{|c|l|c|}
\hline \multicolumn{1}{|c|}{ Table 1. Waste Form Groups (2 pages) } & Abbreviation \\
\hline Name & \multicolumn{1}{|c|}{ Description } & LIQ \\
\hline $\begin{array}{c}\text { Mixuid } \\
\text { Saltcake w/o liquid }\end{array}$ & $\begin{array}{l}\text { At least 97 volume \% liquids. } \\
\text { volume \% mixture of sludge and saltcake. Liquid layer less than 1 } \\
\text { meter thick. }\end{array}$ & MIX-NL \\
\hline $\begin{array}{c}\text { Saltcake/Salt Slurry } \\
\text { w/ liquid }\end{array}$ & $\begin{array}{l}\text { Greater than 3 volume \% solids; solids composition greater than or } \\
\text { equal to 70 volume \% saltcake/salt slurry. Liquid layer greater than 1 } \\
\text { meter thick. }\end{array}$ & SC/SS-LIQ \\
\hline $\begin{array}{c}\text { Saltcake/Salt Slurry } \\
\text { w/o liquid }\end{array}$ & $\begin{array}{l}\text { Greater than 3 volume \% solids; solids composition greater than or } \\
\text { equal to 70 volume \% saltcake/salt slurry. Liquid layer less than 1 } \\
\text { meter thick. }\end{array}$ & SC/SS-NL \\
\hline
\end{tabular}

\footnotetext{
${ }^{(3)}$ Excel is a registered trademark of Microsoft Corporation
} 
Table 1. Waste Form Groups (2 pages)

\begin{tabular}{|c|l|c|}
\hline Name & \multicolumn{1}{|c|}{ Description } & Abbreviation \\
\hline Sludge w/ Liquid & $\begin{array}{l}\text { Greater than 3 volume \% solids; solids composition greater than or } \\
\text { equal to } 70 \text { volume \% sludge. Liquid layer greater than 1 meter } \\
\text { thick. }\end{array}$ & SL-LIQ \\
\hline Sludge w/o Liquid & $\begin{array}{l}\text { Greater than } 3 \text { volume \% solids; solids composition greater than or } \\
\text { equal to } 70 \text { volume \% sludge. Liquid layer less than 1 meter thick. }\end{array}$ & SL-NL \\
\hline
\end{tabular}

For each waste form groups that contained a missing value for any of the aforementioned constituents, a default number value was assigned based on the available data from other tanks within the same subset. The default number, a highlighted negative value (see Table 2 \& Table 3 ), is a placeholder that associates a particular distribution function (see Table 4) for the missing data value.

The default distributions are based on the waste form subsets and developed via a statistical analysis software program, Crystal Ball ${ }^{\circledast}$. Crystal Ball ${ }^{\circledR}$ is a Microsoft Excel ${ }^{\circledR}$ add-in tool that uses Monte Carlo simulation to help analyze the risks and uncertainties associated with spreadsheet models. From the defined distributions, the normal space mean value (average) is selected to represent the default distribution value for all tanks without data in the specific waste form type and group.

\subsection{Assumptions}

The assumption is that the data contained within the Tank Waste Information Network has been checked and reviewed to insure that all values are accurate and any inconsistencies are documented. It is also assumed that the values represented in the various databases are the most current and best version of all available data.

\subsection{Calculations \& Input Data}

For the components, $\mathrm{Al}, \mathrm{NO}_{2}, \mathrm{NO}_{3}$ and $\mathrm{TOC}$, the units are reported in micrograms per gram $(\mu \mathrm{g} / \mathrm{g})$. The values are multiplied by the layer densities $(\mathrm{g} / \mathrm{ml})$ to obtain the desired units, micrograms per milliliter $(\mu \mathrm{g} / \mathrm{ml})$. Equation 1 and 2 are examples for aluminum.

$$
\operatorname{Conc}_{\mathrm{Al}} \cdot \frac{\mu \mathrm{g}}{\mathrm{ml}} \square \text { Density } \frac{\mathrm{g}}{\mathrm{ml}} \times \operatorname{Conc}_{\mathrm{Al}} \cdot \frac{\mu \mathrm{g}}{\mathrm{g}} \quad \text { Equation } 1
$$

The total concentration of the constituents listed along with total density in each tank is calculated as a volume ratio of the concentration in the layers versus the total volume of the tank:

$$
\operatorname{Conc}_{\mathrm{Al}}=\frac{\left[\left(\text { Conc }_{\mathrm{Al}} \times \mathrm{V}_{\mathrm{Al}}\right)+\left(\text { Conc }_{\mathrm{Al}} \mathrm{SC}_{\mathrm{SC}} \times \mathrm{V}_{\mathrm{Al}}\right)+\left(\operatorname{Conc}_{\mathrm{SC}}\right) \times \mathrm{V}_{\mathrm{Al}}\right)}{\left(\mathrm{V}_{\mathrm{Al}}+\mathrm{V}_{\mathrm{Al}}+\mathrm{V}_{\mathrm{Sl}}\right)}
$$


RPP - 6069 Rev. 0

For the radionuclides $\mathrm{Cs}^{137} \& \mathrm{Sr}^{90}$, the reported values were already in the desired units, microcuries per gram $(\mu \mathrm{Ci} / \mathrm{g})$. To determine the total tank concentrations these values must be ratioed on a mass weighted average basis, as shown by the following equation:

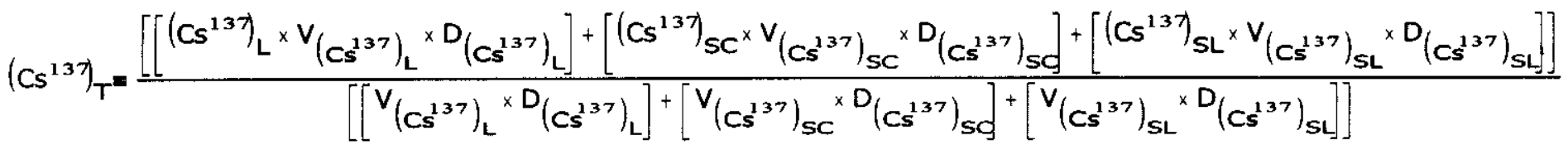

Equation 3

All the reported $\mathrm{BBCD}$ and calculated total concentration values were compiled, Table 2, with the desired input data points that are missing represented by highlighted cells containing a default placeholder number $(-8 X X)$. Table 3 contains the TCD data for the weight percent $\mathrm{H}_{2} \mathrm{O}$, also with default placeholder numbers for nonexistent values. 
RPP - 6069 Rev. 0

This page intentionally left blank 







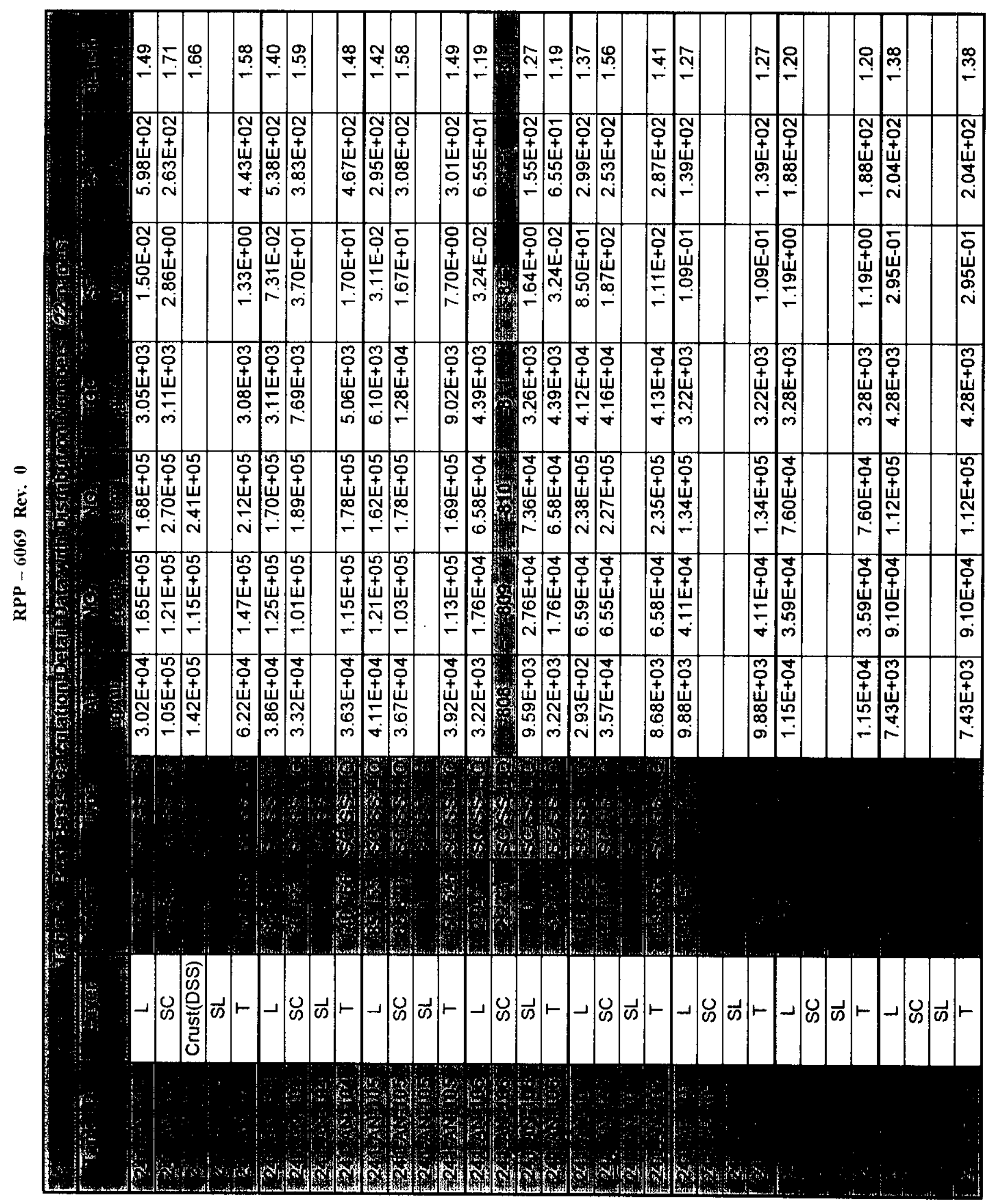









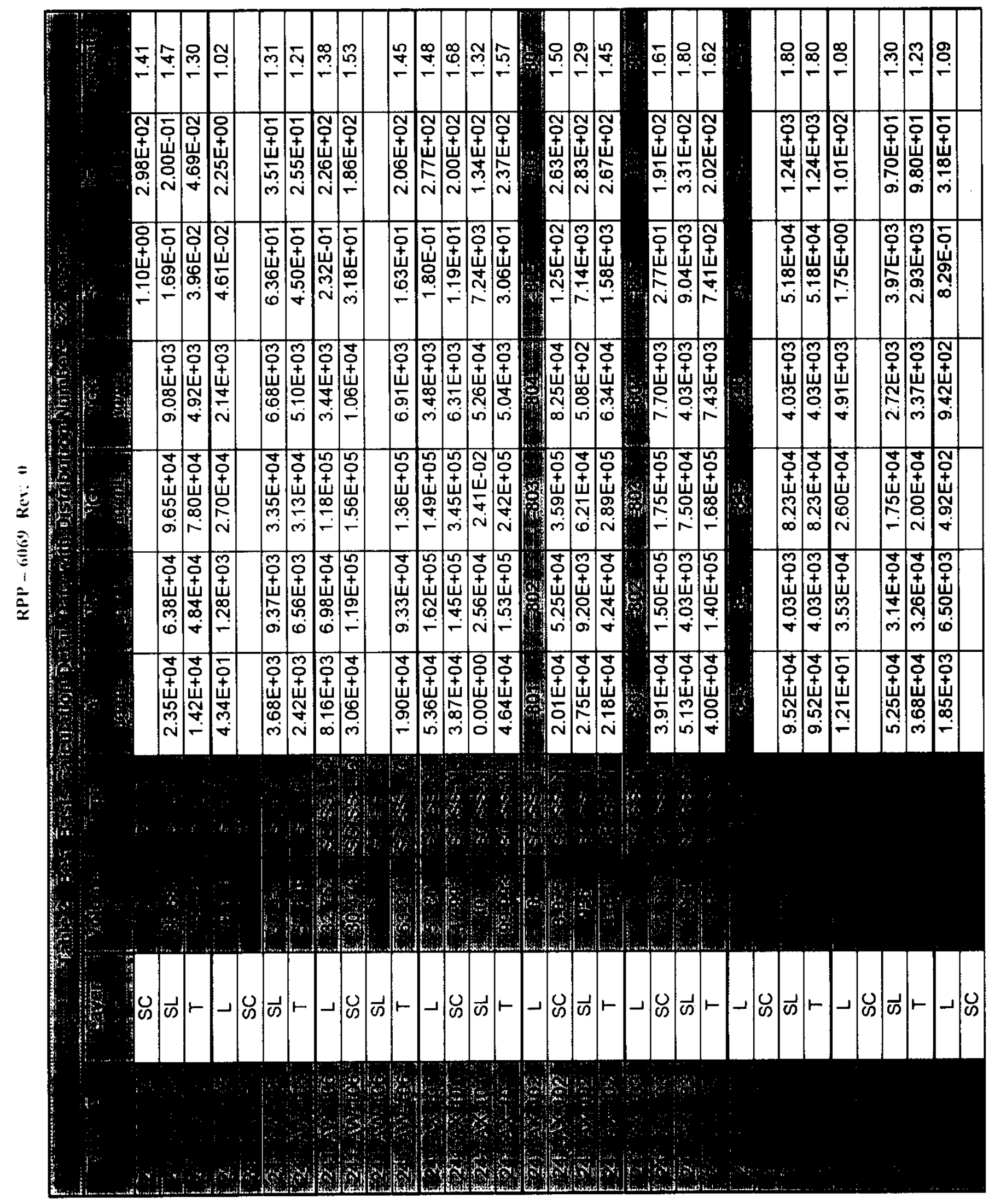




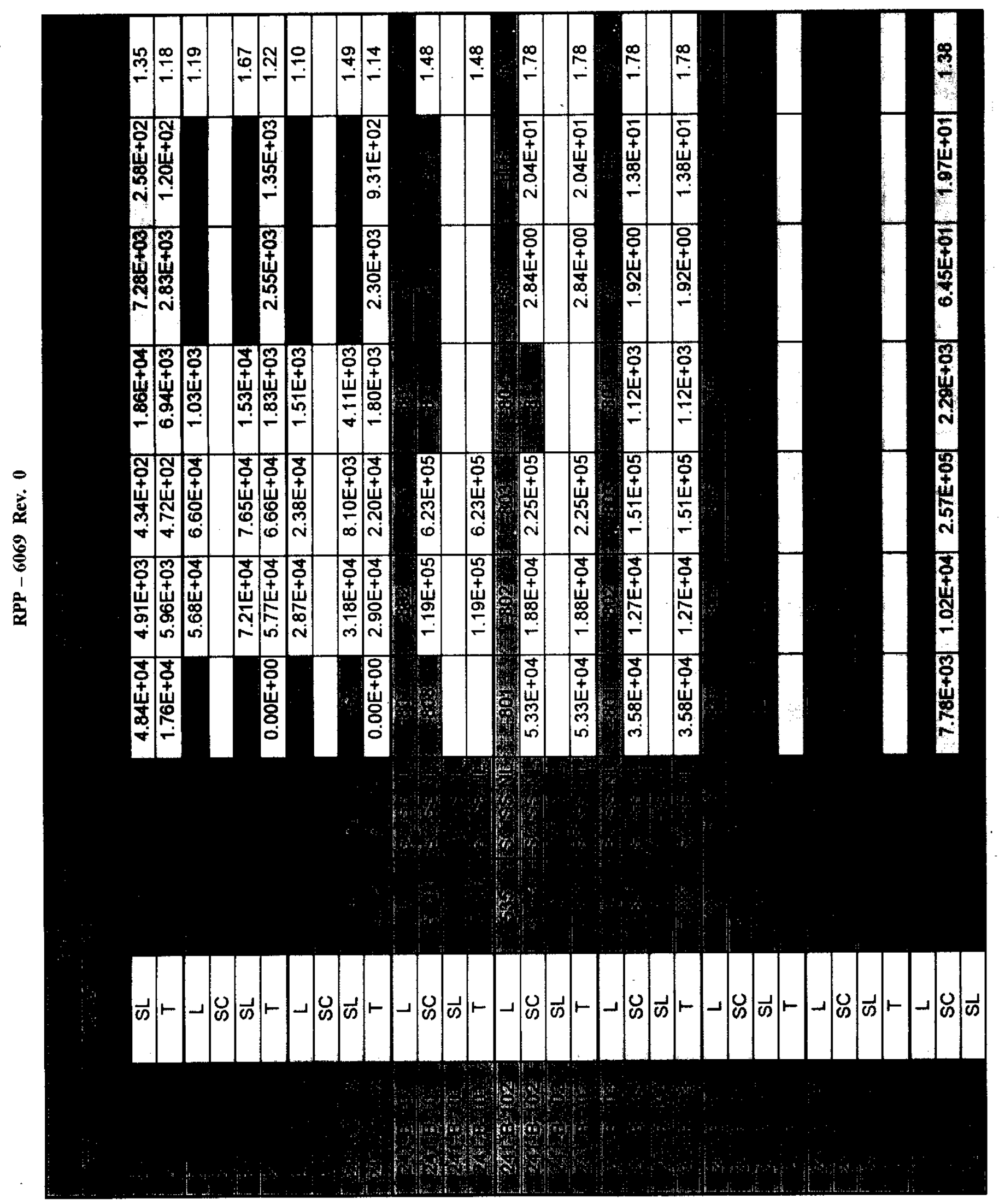




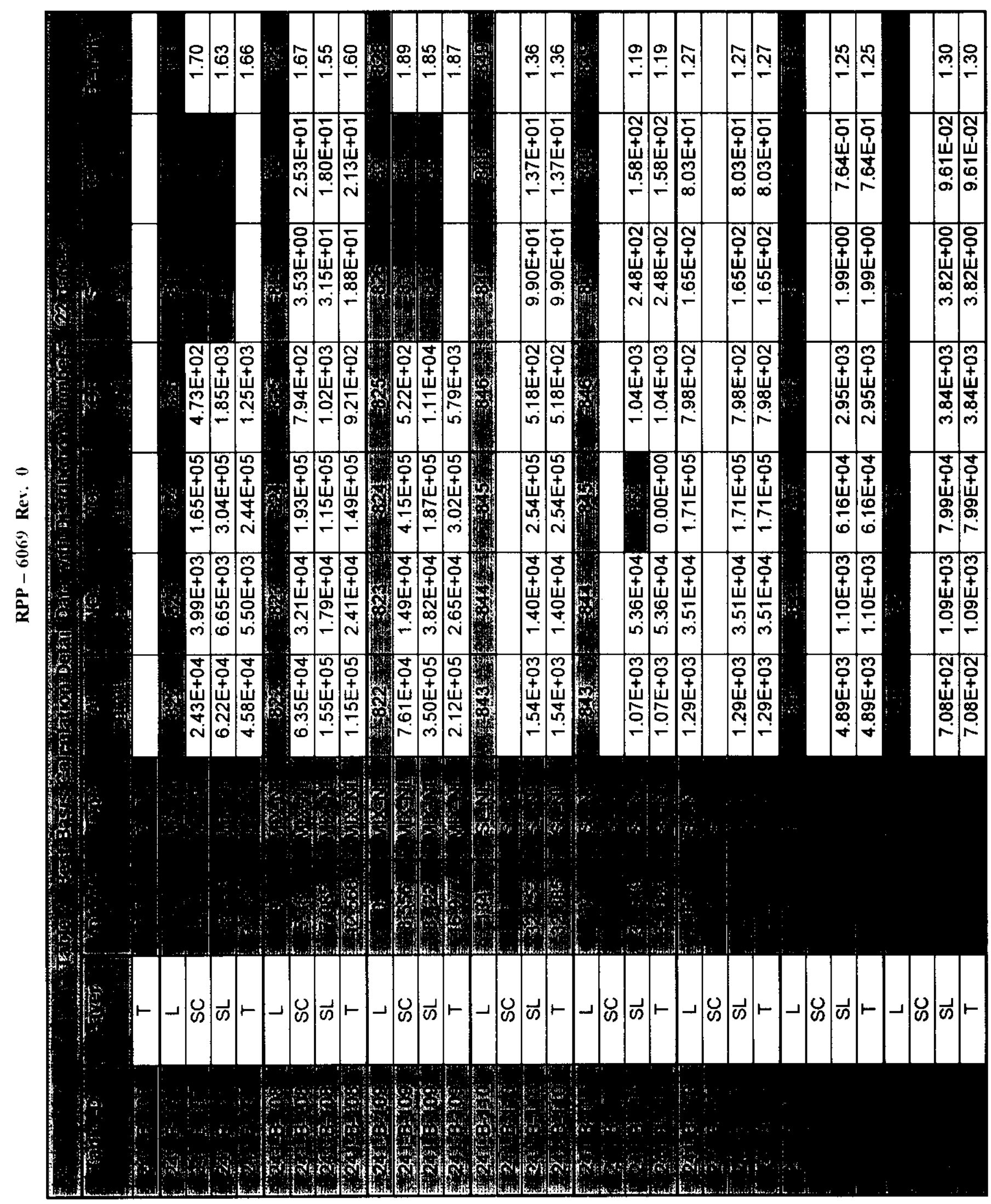




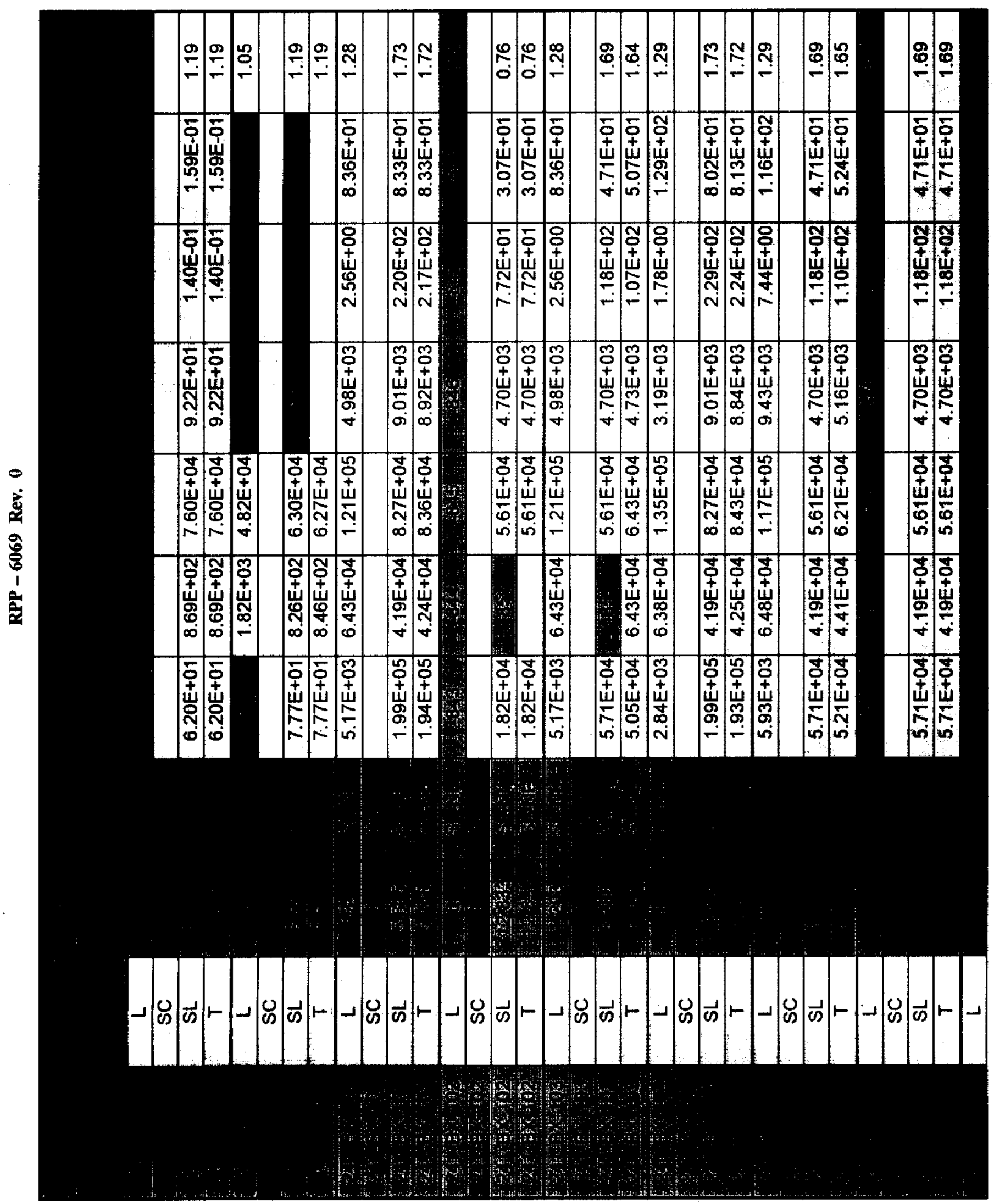









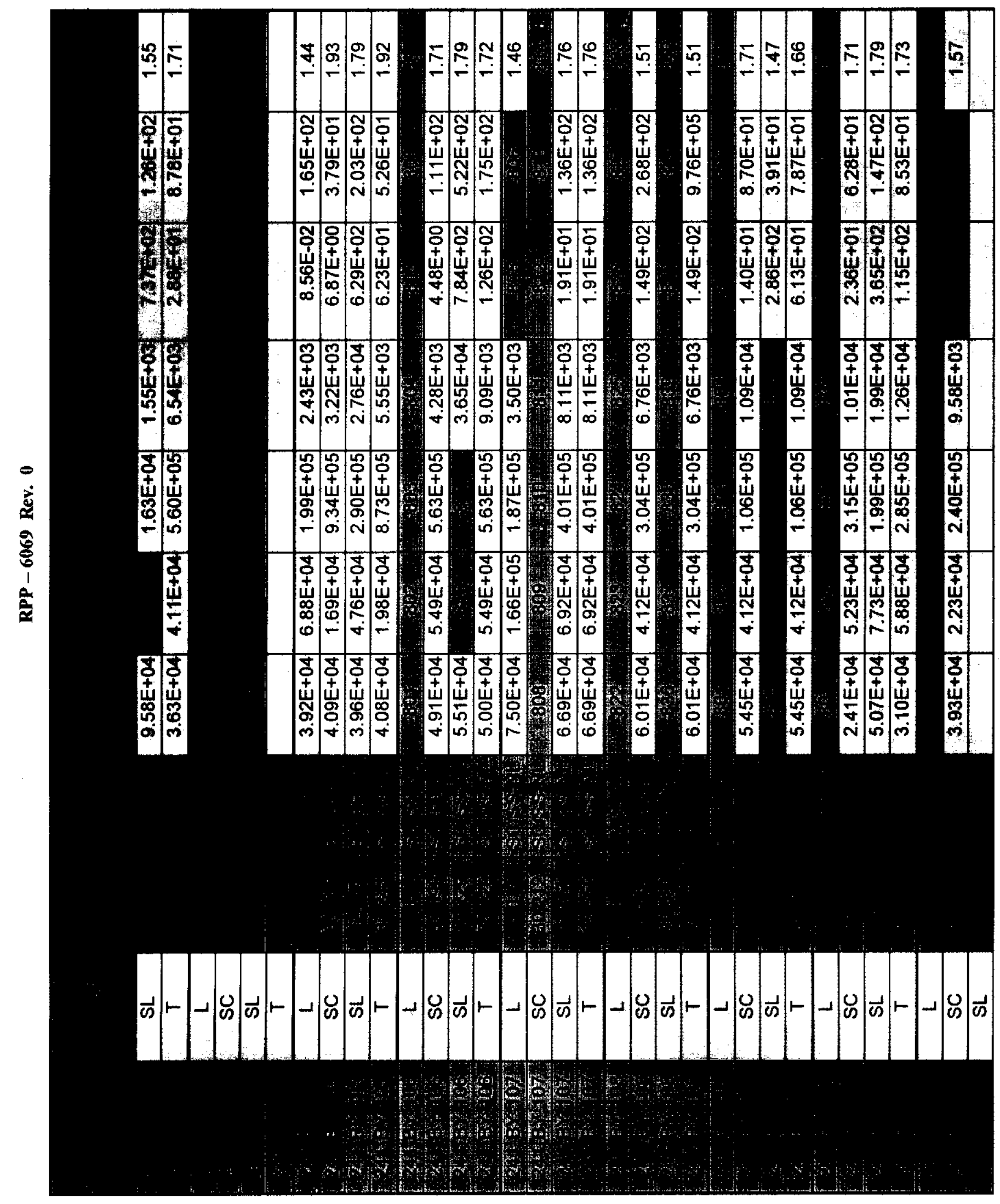




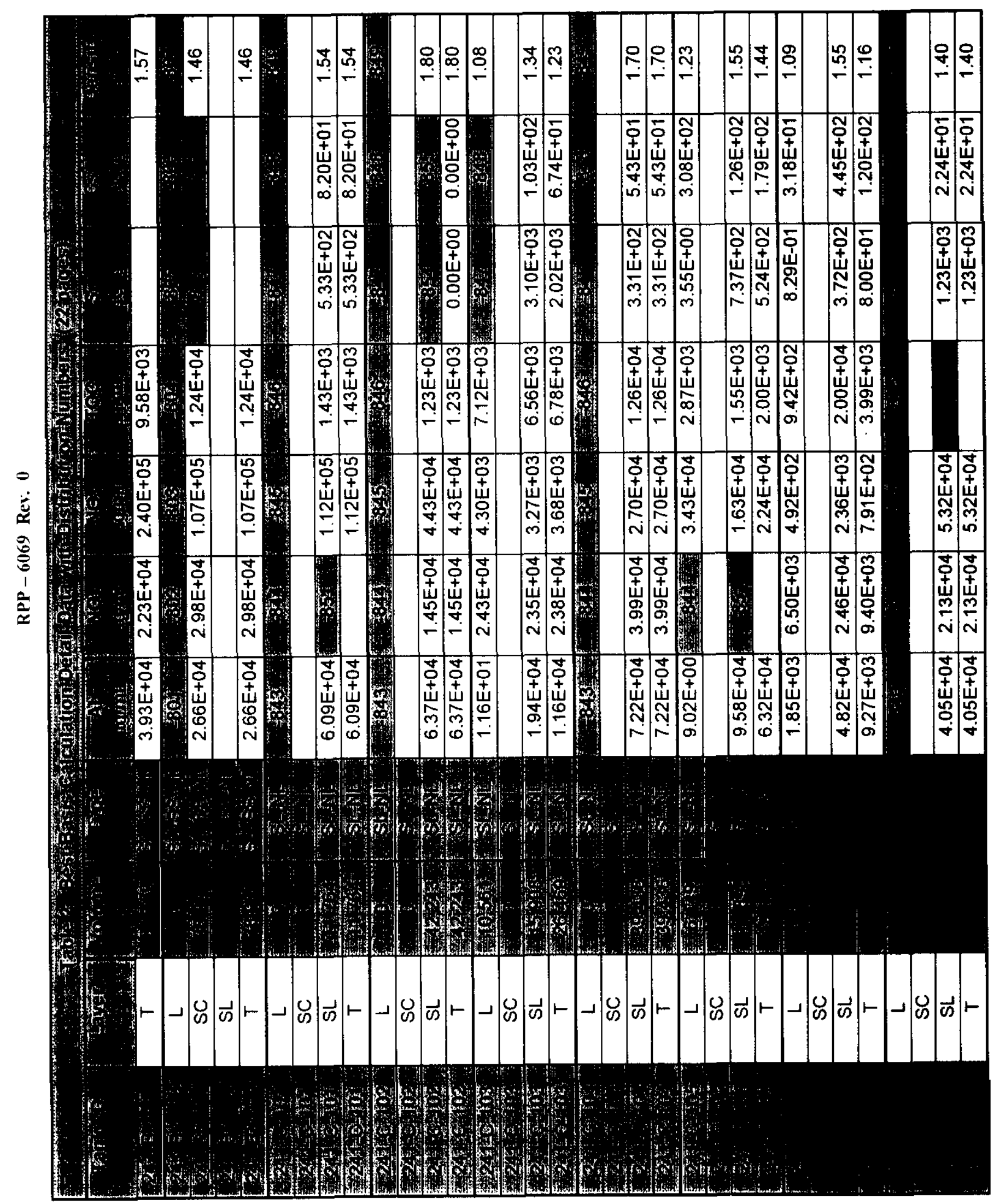




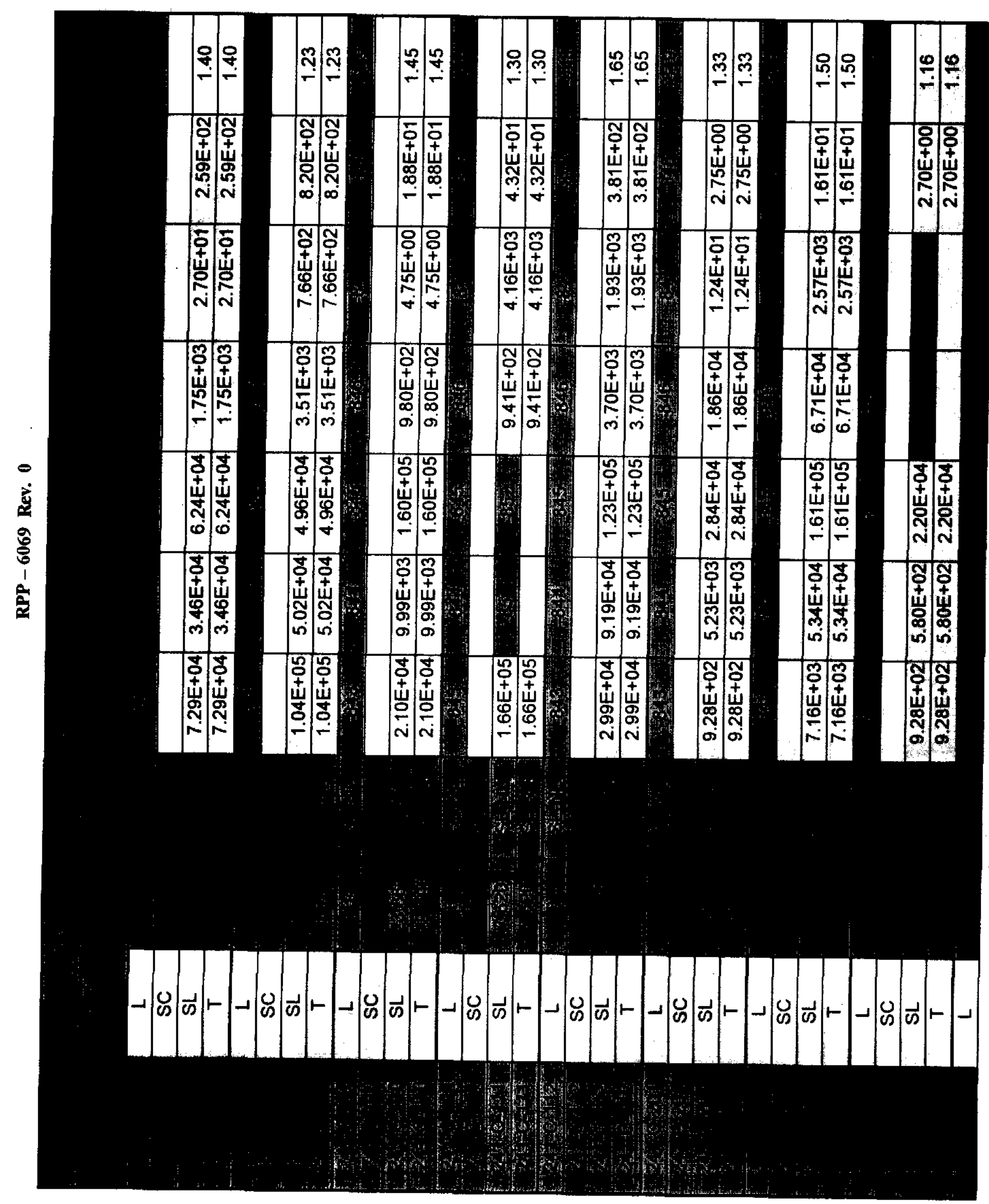




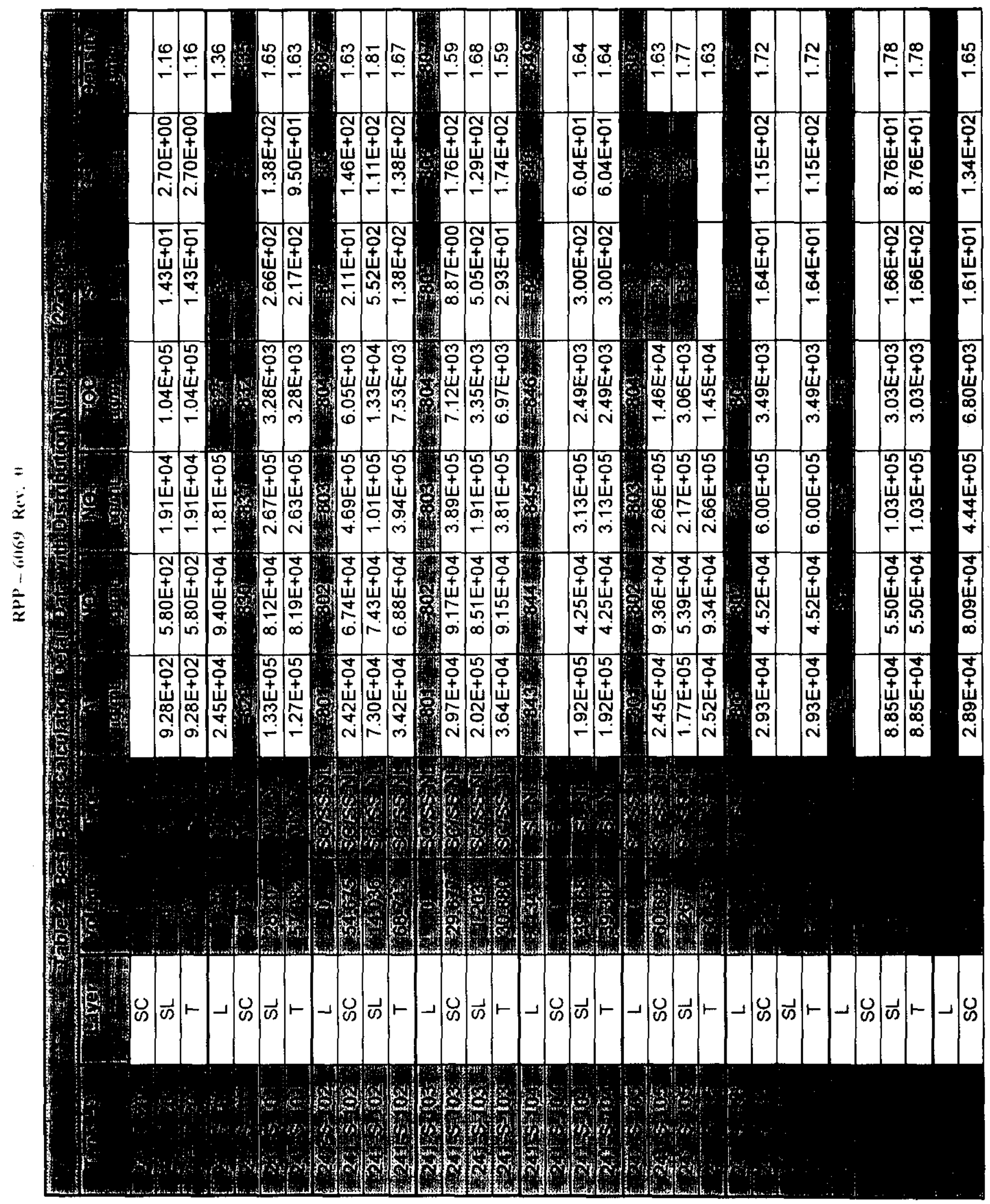




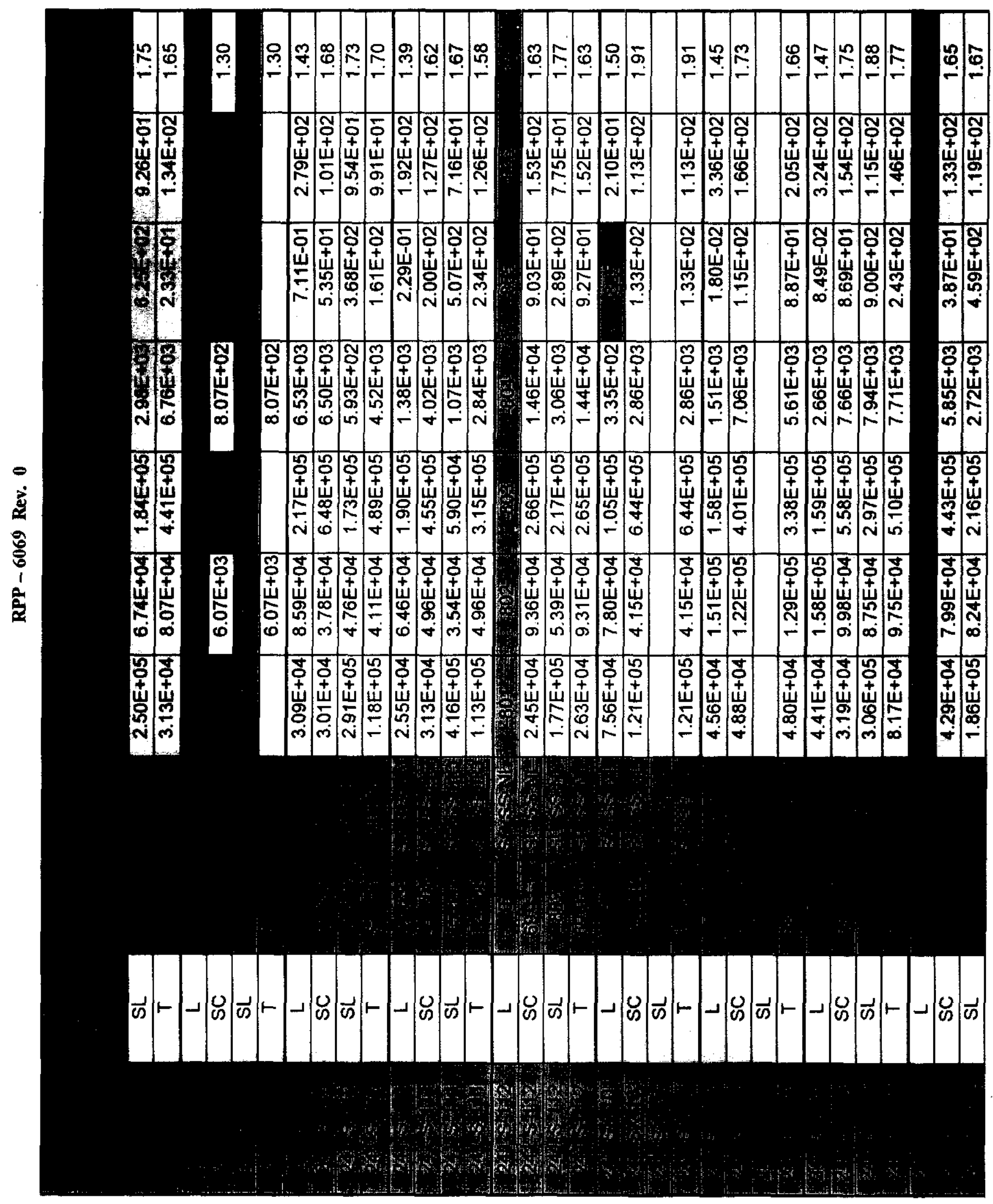




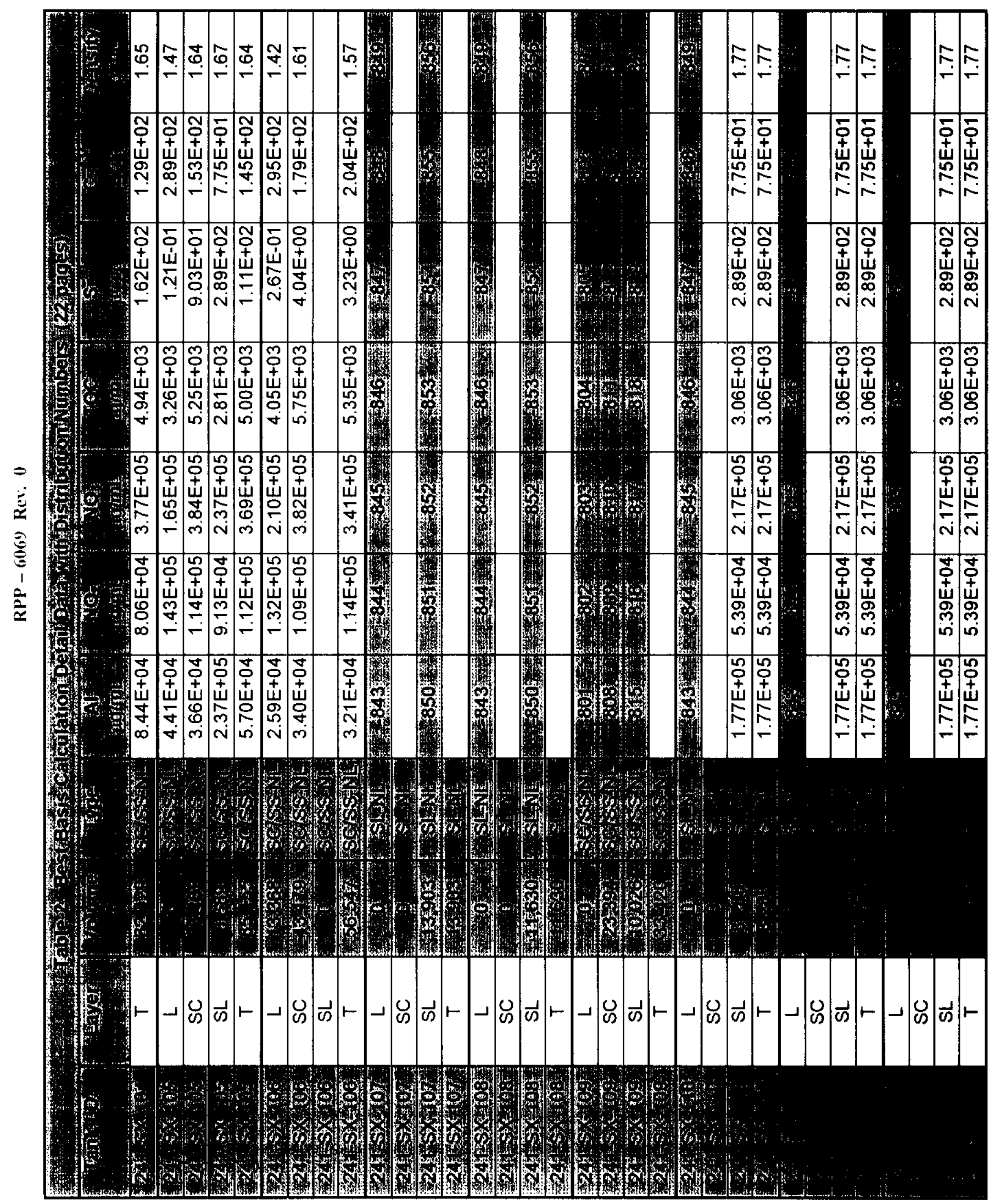




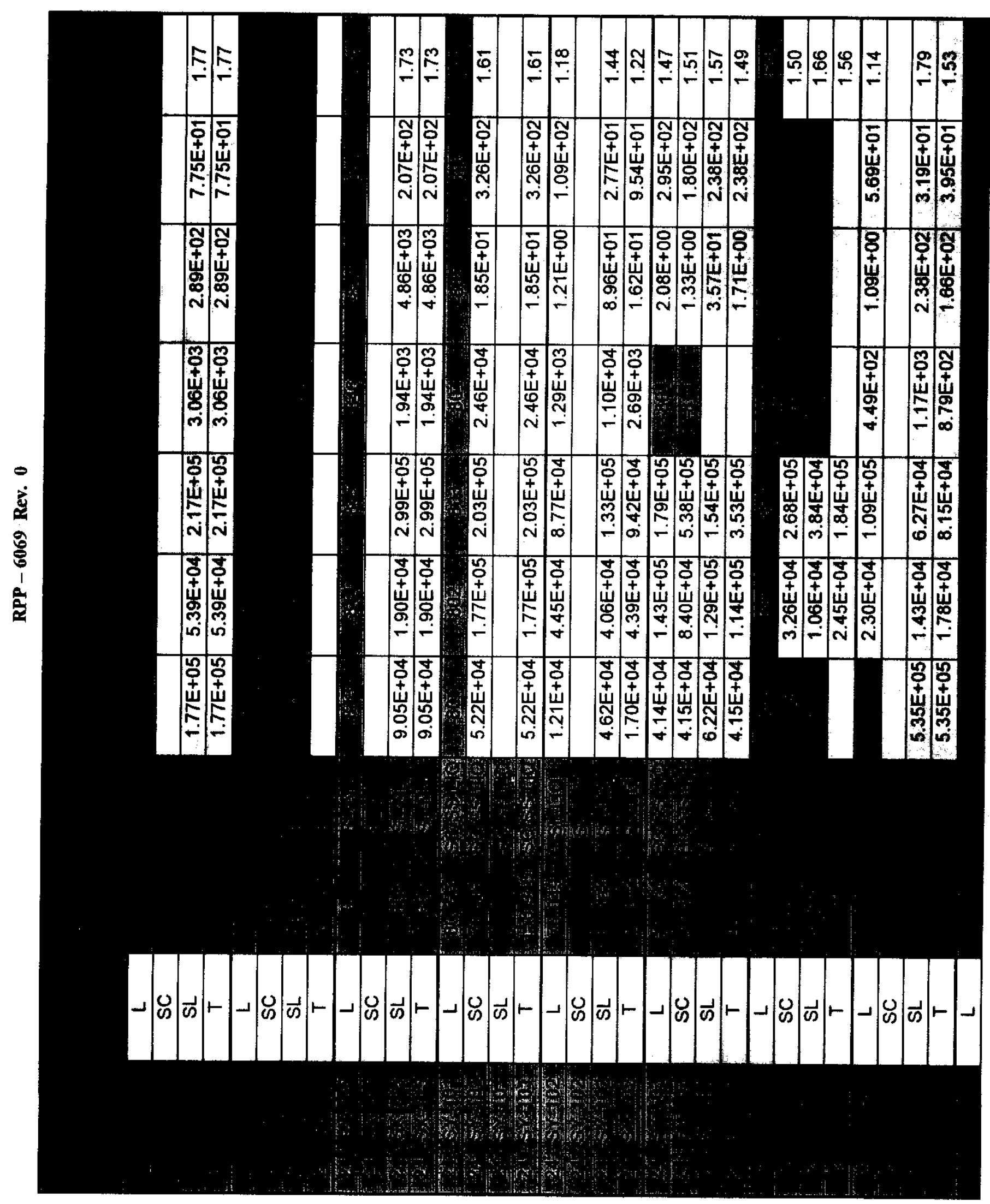




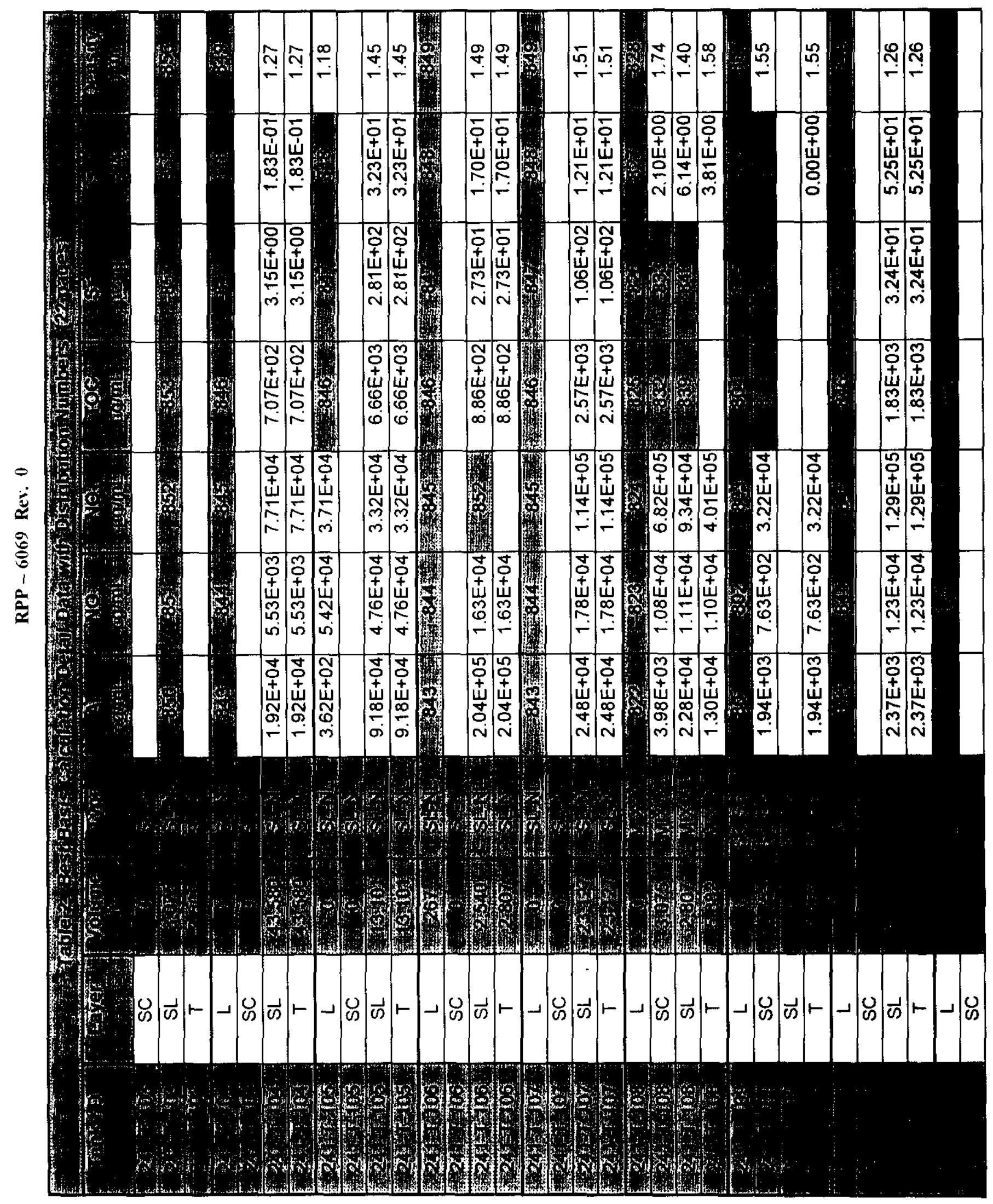




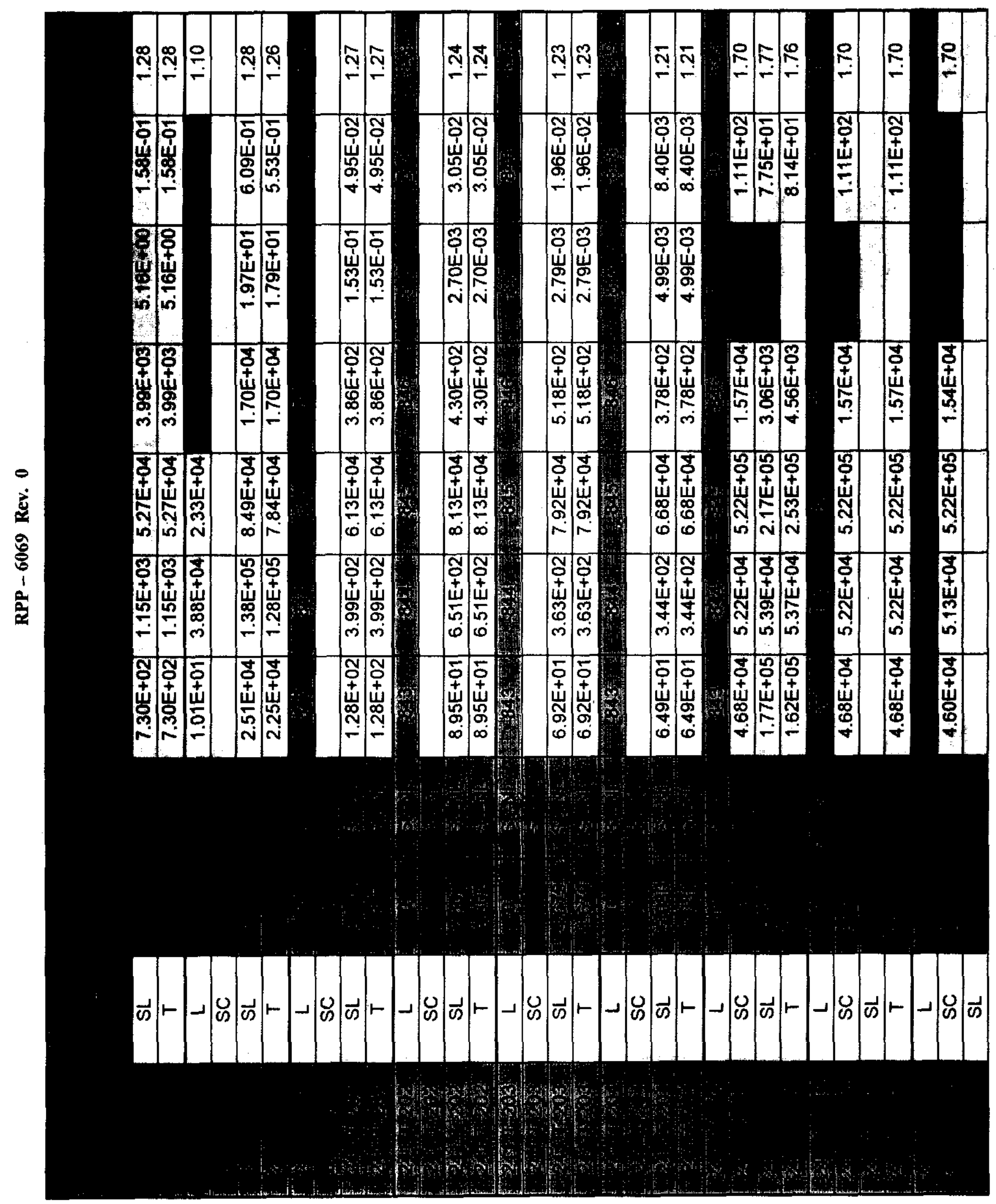









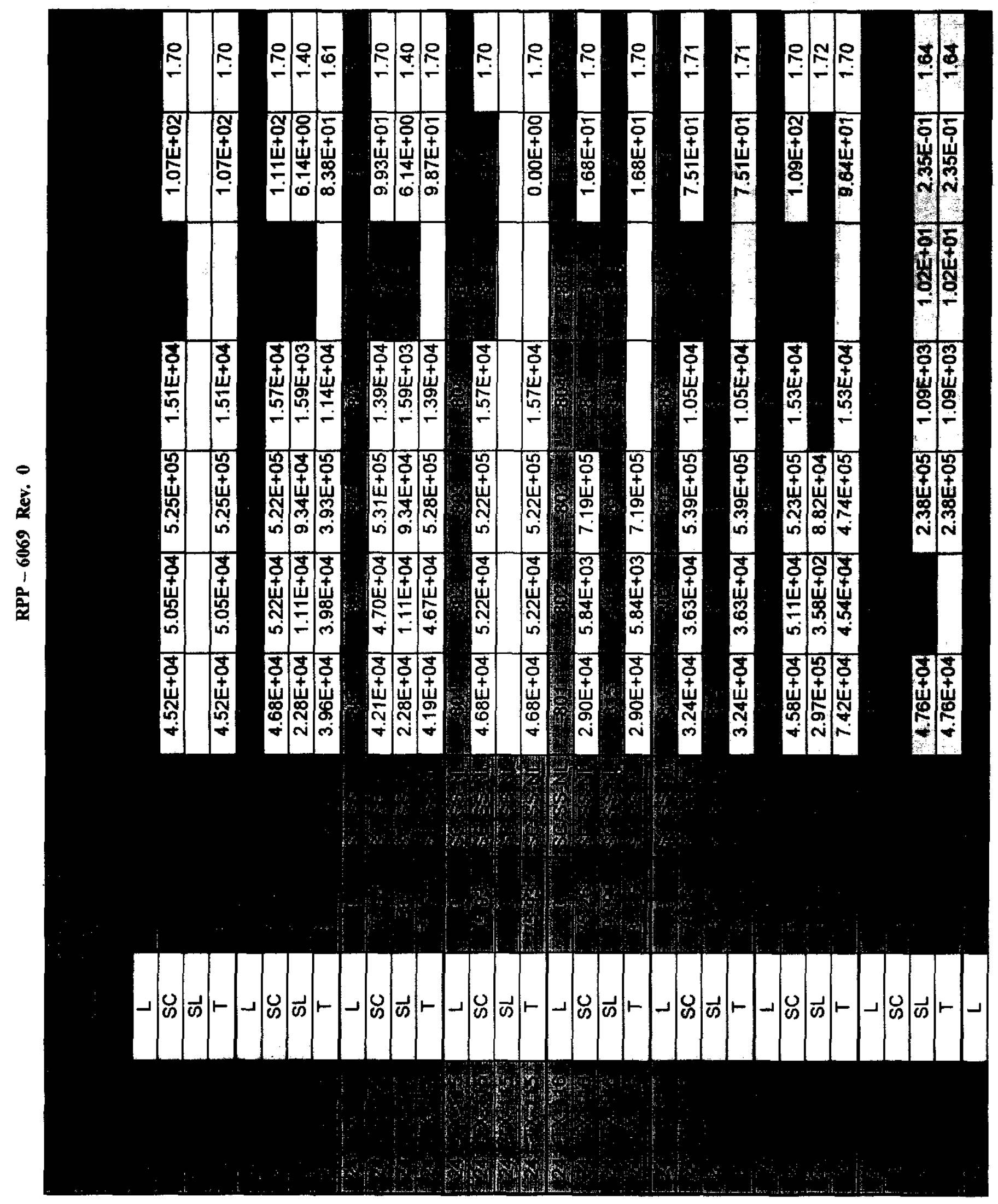




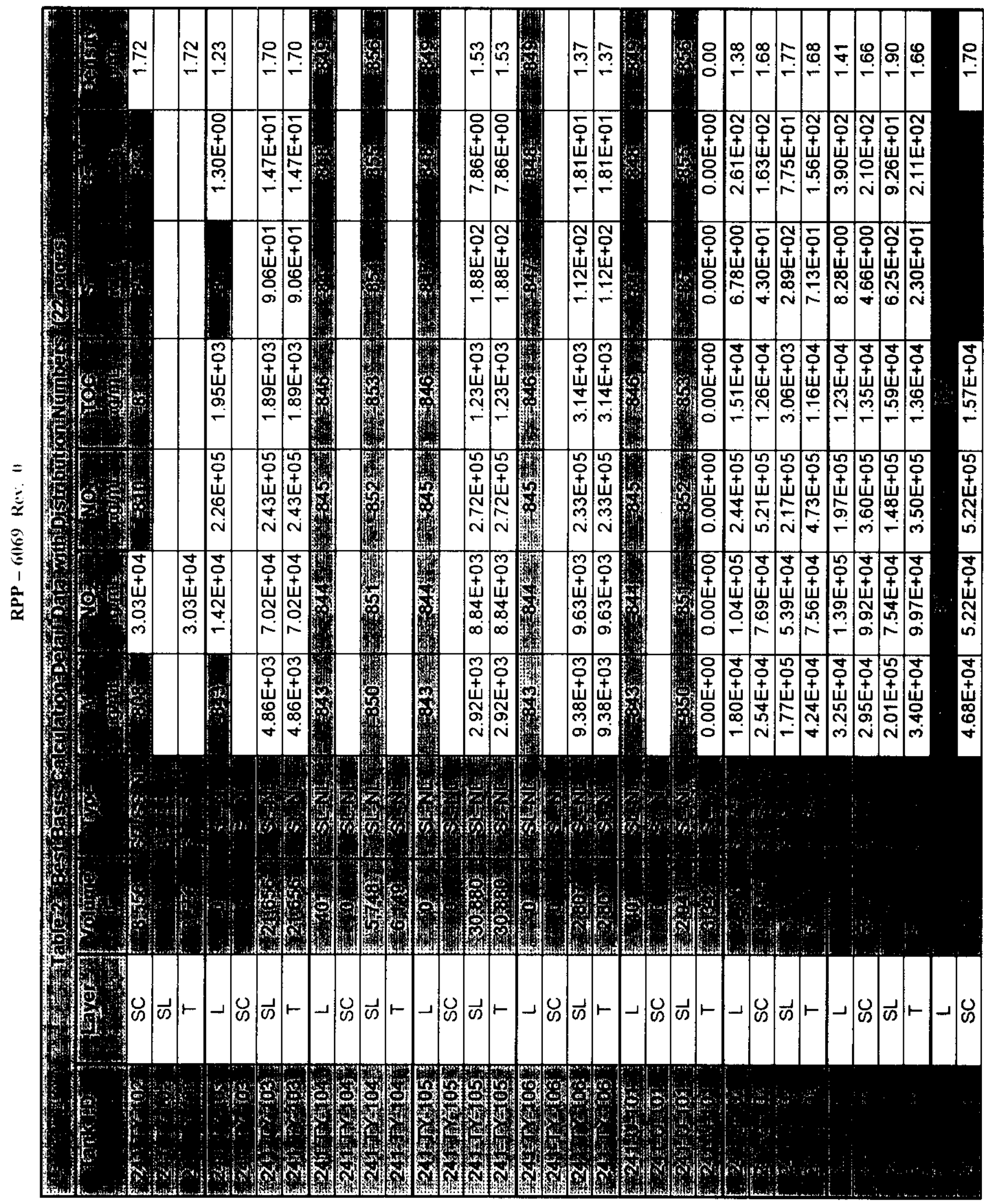




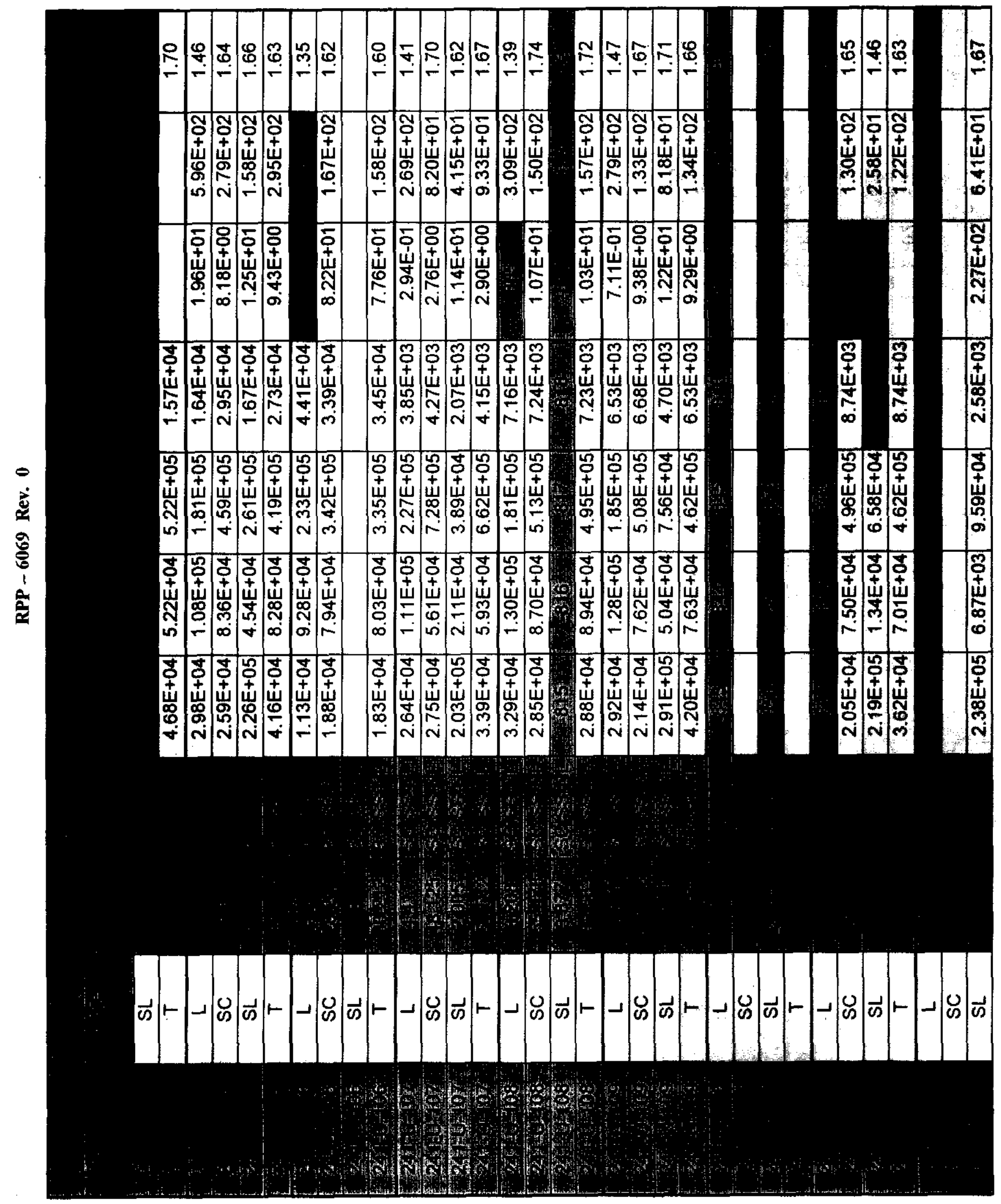




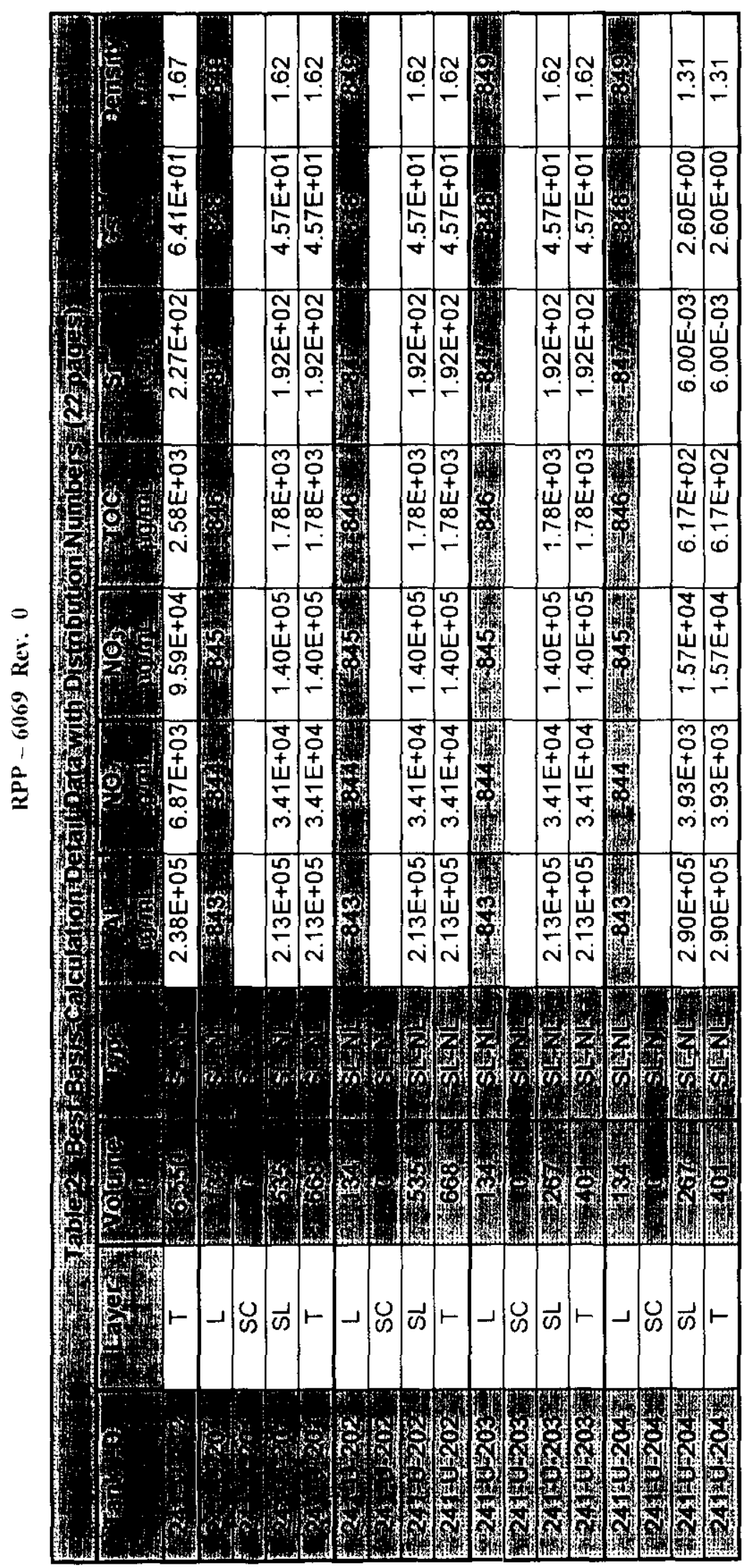




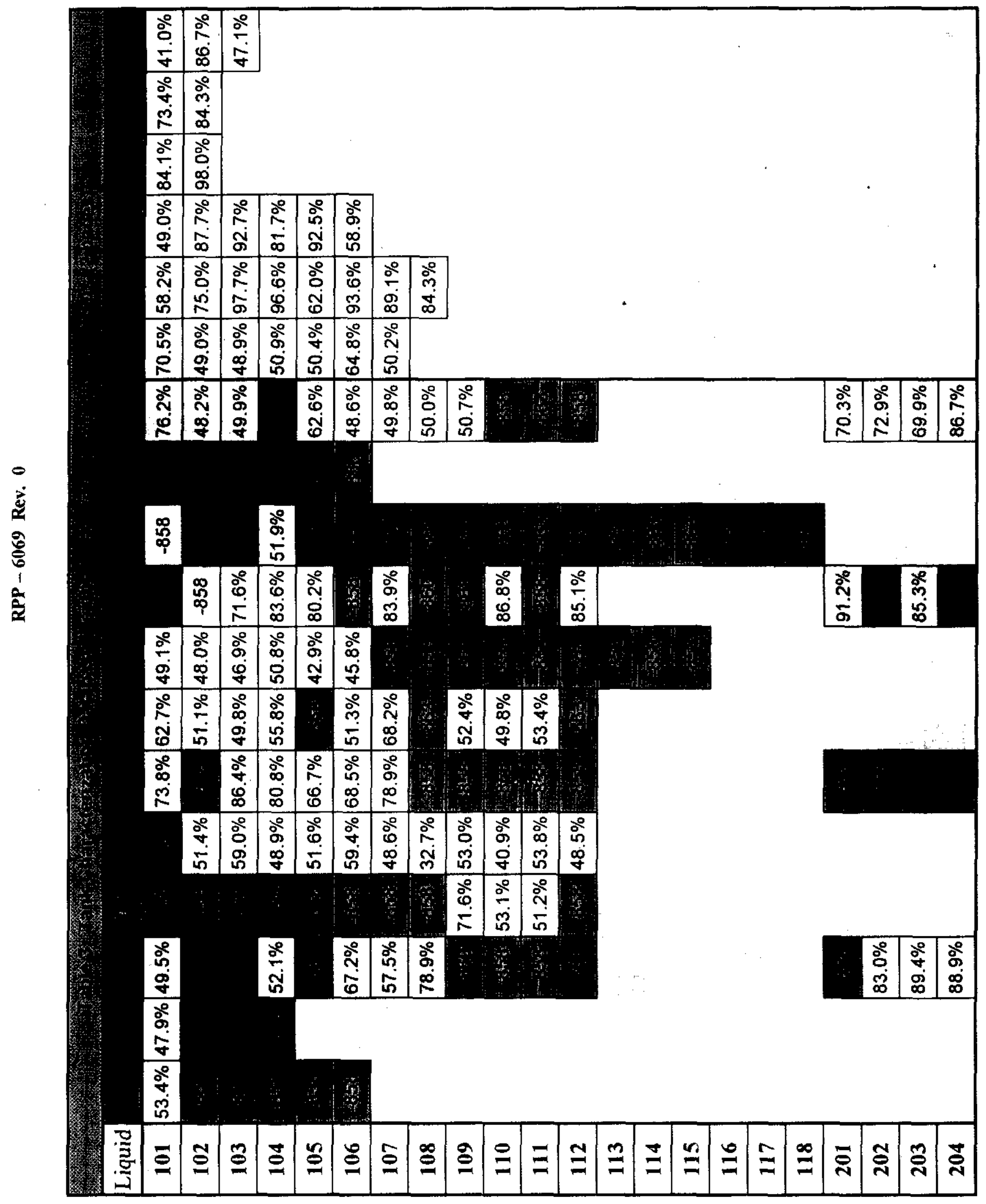




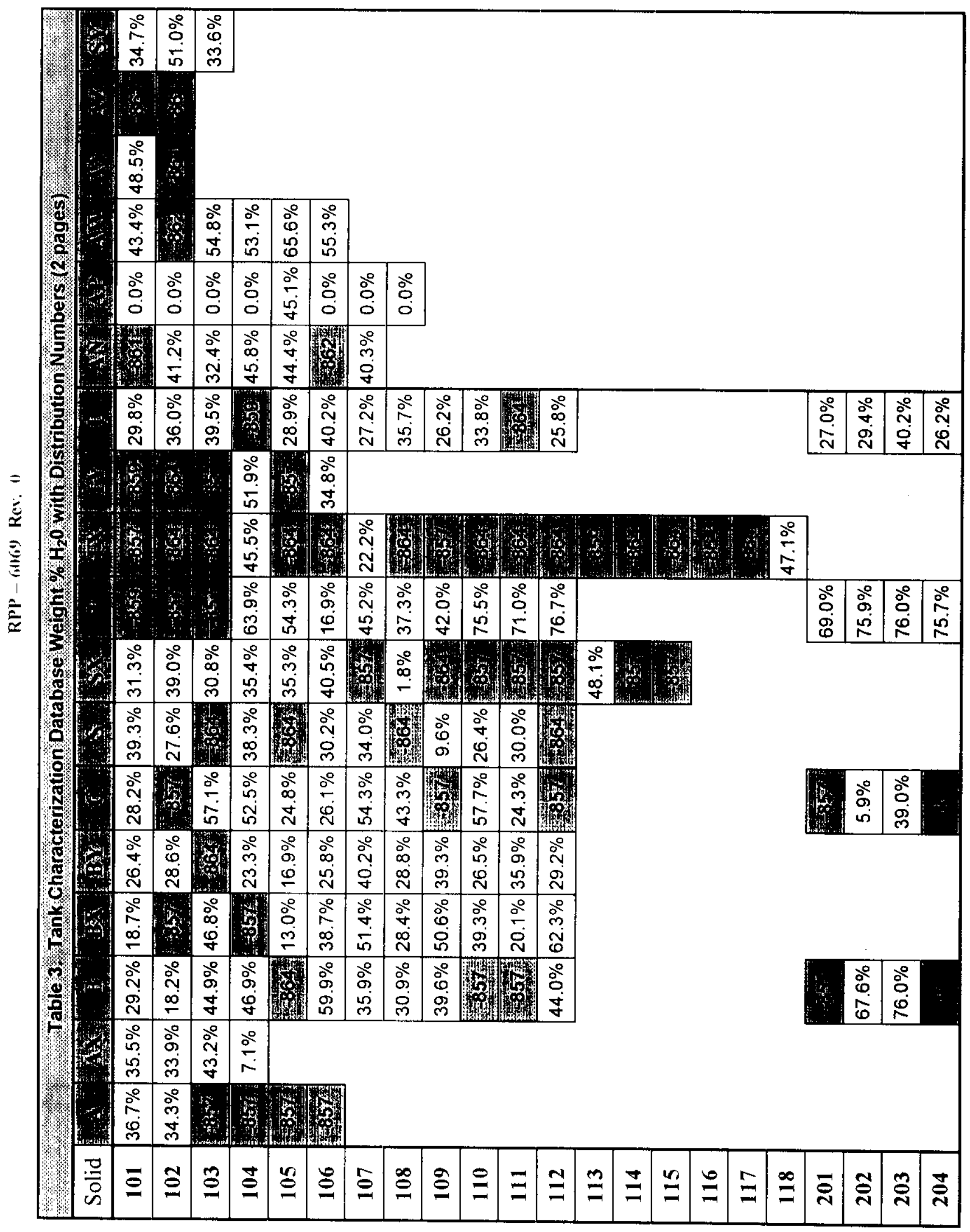


The tanks were categorized by waste group type and layer definitions and then sorted by the individual constituents for assessment. These waste group subsets are then assigned a distribution number value, corresponding to the default number $(-8 \mathrm{XX})$ in the previous tables, for further analysis. For the tanks with missing data in one or more constituents, statistical analysis methods were utilized to develop reasonable values.

To use the statistical analysis program, Crystal Ball ${ }^{\circledast}$, initial assumption and forecast values are chosen which allows the software to select the optimum distribution. For this study the possible distributions were limited to five that were easily reproducible; the normal, lognormal, exponential, triangular and linear distributions. Once the best possible distribution was chosen to fit the waste group data, the input conditions were adjusted to remove negative values and recorded in the spreadsheet (Figure 1).

\begin{tabular}{|c|c|c|c|c|}
\hline & & \multicolumn{3}{|c|}{ SCISS - LIQ $\leftarrow$ waste group type } \\
\hline$:$ & i & Assumption & \multicolumn{2}{|c|}{$25,000 \leftarrow$ Input value, an initial guess for the Crystal Ball $(C B)$ program } \\
\hline$\therefore \quad i$ & $1.51 E+04$ & \multirow[b]{2}{*}{ Forecast } & \multirow{2}{*}{\multicolumn{2}{|c|}{$25,000 \bigcirc$ Cell value equal to Assumption value cell }} \\
\hline$\therefore \quad \cdots$ & $3.02 E+04$ & & & \\
\hline - & $3.86 E+04$ & \multirow{2}{*}{\multicolumn{3}{|c|}{ Normal Distribution $\leftarrow$ Optimum distribution type evaluated from dataset by $\mathrm{CB}$ program }} \\
\hline$\therefore \quad i$ & $4.11 E+04$ & & & \\
\hline$: \cdots \quad \therefore$ & $3.22 E+03$ & \multicolumn{2}{|l|}{$\log$ mean } & \multirow{11}{*}{$\begin{array}{l}\text { Initial probability distribution parameters from CB } \\
\text { program, dependent on type of distribution chosen. }\end{array}$} \\
\hline$\therefore \quad \therefore:$ & $2.93 E+02$ & \multicolumn{2}{|l|}{$\log$ stddev } & \\
\hline$\cdots n$ & $2.57 E+04$ & $\min$ & & \\
\hline$\therefore \quad \therefore$ & $3.21 E+04$ & $\max$ & 72,940 & \\
\hline and & $1.09 \mathrm{E}+03$ & \multicolumn{2}{|l|}{ likeliest } & \\
\hline for ond & $8.16 E+03$ & mean & 26,623 & \\
\hline 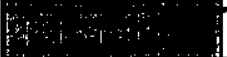 & $2.55 E+04$ & stddev & 14,763 & \\
\hline 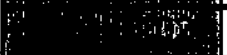 & $4.56 E+04$ & \multicolumn{2}{|l|}{ rate } & \\
\hline a ond & $4.14 \mathrm{E}+04$ & \multirow{2}{*}{\multicolumn{2}{|c|}{. }} & \\
\hline W & $4.46 \mathrm{E}+04$ & & & \\
\hline En a & $5.36 E+04$ & \multirow{10}{*}{\multicolumn{3}{|c|}{$\begin{array}{l}\uparrow \text { Number placeholder associated to the values calculated from the } \\
\text { above distribution type and initial conditions for the waste group and layer of } \\
\text { interest. }\end{array}$}} \\
\hline mon & $2.59 E+04$ & & & \\
\hline 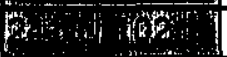 & $1.80 E+04$ & & & \\
\hline$(5)$ if & $3.25 E+04$ & & & \\
\hline Et & $2.98 E+04$ & & & \\
\hline lods ando & $1.13 E+04$ & & & \\
\hline os a n & $2.64 E+04$ & & & \\
\hline Fot & $3.29 E+04$ & & & \\
\hline 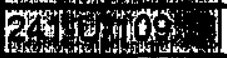 & $2.92 E+04$ & & & \\
\hline$\uparrow$ Tank id & & values & & \\
\hline
\end{tabular}

Figure 1. Dataset for Distribution Analysis, Input Conditions and Distribution Number.

The statistical software utilizes a random number generator along with the optimum distribution and initial probability distribution parameters (Table 4) to forecast a frequency chart (Figure 2) and distribution tables (Figure 3) that will be utilized in determining values for the missing waste tank data. 
RPP - 606, Rev. 1

This page intentionally left blank 
ֻ † f

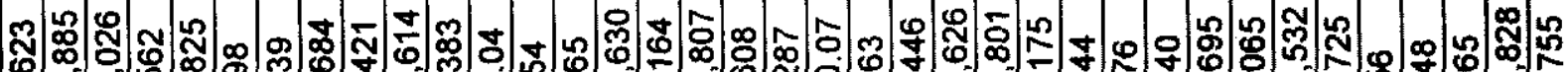
N|

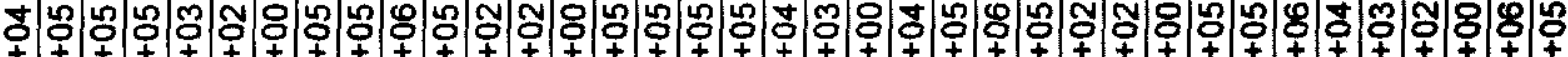

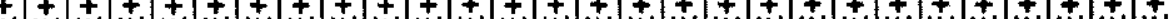

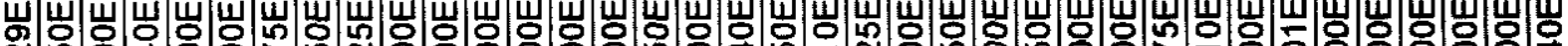
N̦

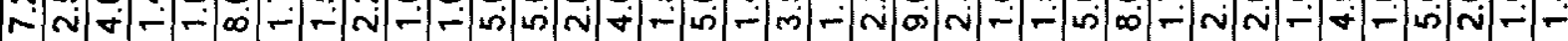

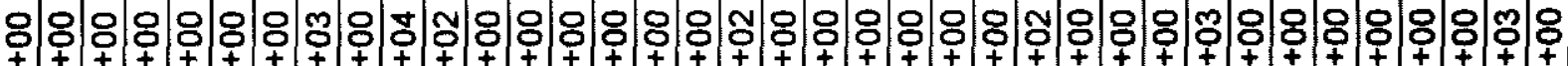

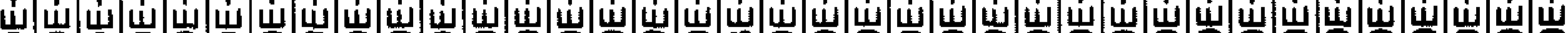

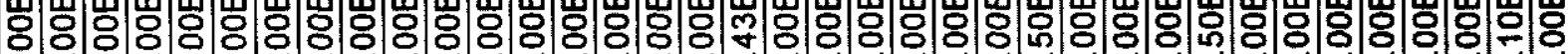

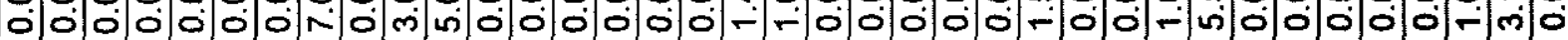

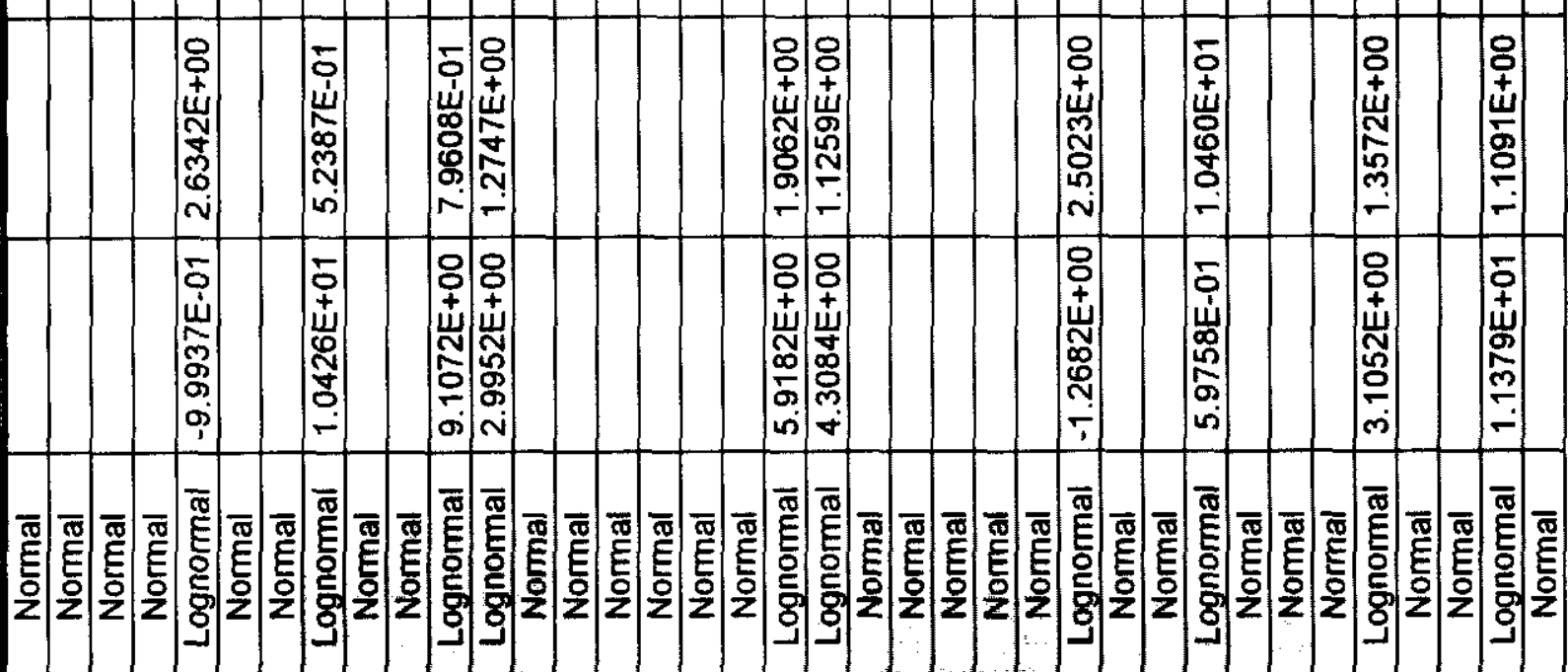







Output chart and values from the Crystal Ball ${ }^{\circledR}$ program

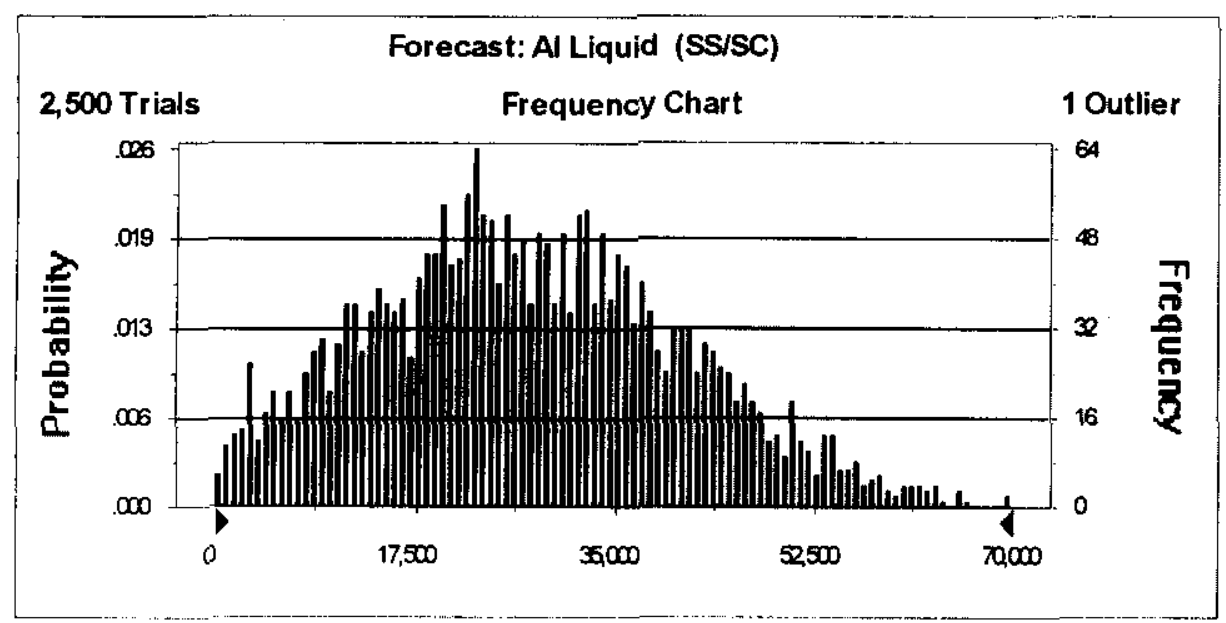

Figure 2. Frequency Chart Output from Crystal Ball (CB) Statistical Analysis Software.

Table -

Forecast: Al Liquid (SS/SC)

Forecast: Al Liquid (SS/SC)

Statistic Value

Trials

Mean

2,500

Percentile Value

Median

27,469

Mode

26,626

Standard Deviation

Variance

13,571
$184,171,904$

$0 \% \quad 5$

Skewness

Kurtosis

Coeff. of Variability

Range Minimum

Range Maximum

Range Width

$10 \% \quad 9,622$

$20 \% \quad 15,046$

$30 \% \quad 19,647$

$40 \% \quad 23,017$

$50 \% \quad 26,626$

0.26

$60 \% \quad 30,597$

$2.59 \quad 70 \% \quad 34,653$

$\begin{array}{lll}0.49 & 80 \% & 39,312\end{array}$

$5 \quad 90 \% \quad 45,598$

Mean Std. Error

70,205

$100 \%$

70,205

Figure 3. Distribution Output of CB Statistical Analysis Software.

The statistic values generated from the Crystal Ball software for all distributions were then compiled into a reference table (Table 5). 
RPP - 6069 Rev. (1

This page left intentionally blank 


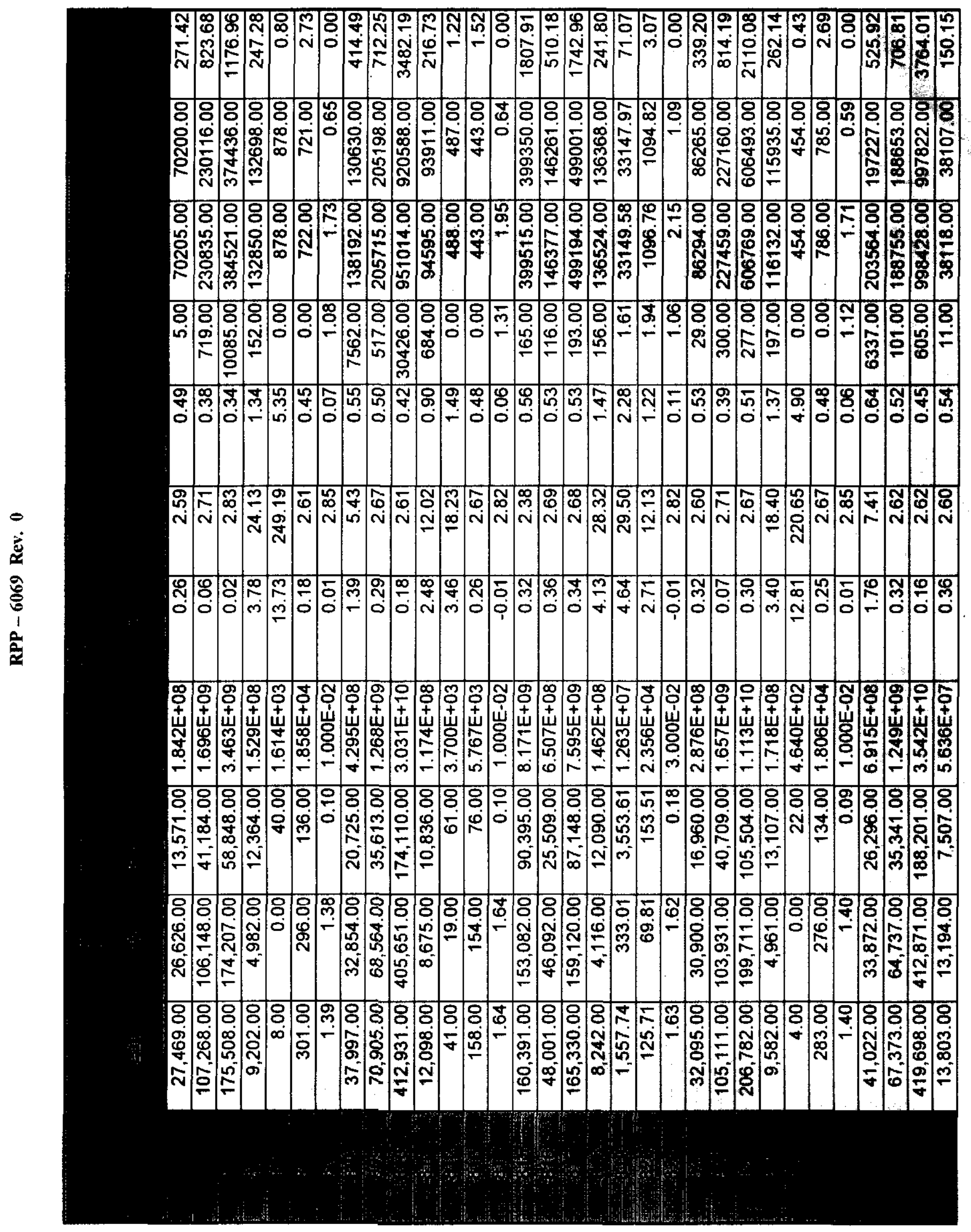




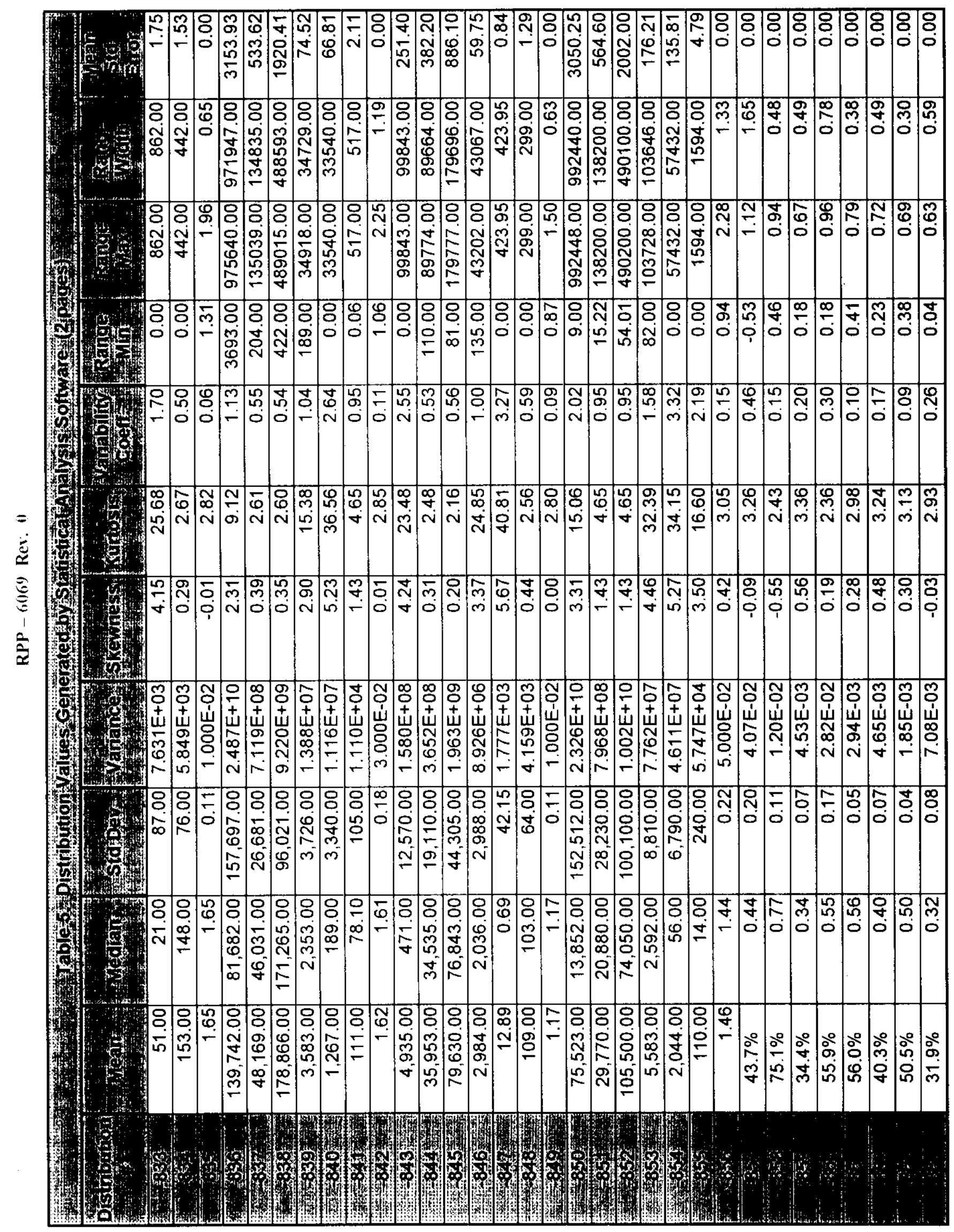


The mean value from Table 5 is then used as the input value for all tanks that do not have other data and fit the waste type and constituent criteria for that distribution.

Once the distribution values are input into Tables 2 and 3, then the new total concentrations can be calculated using the volume or mass weighted ratioing as described in equations $2 \& 3$.

The same type of volume rationing utilized, for the total tank constituents, in equation 2 is also used to convert the salt cake (SC) and sludge (SL) layer concentrations into one solid layer concentration value for each tank:

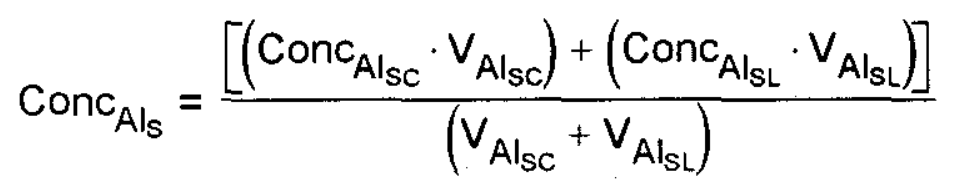

Equation 4

The radionuclides are ratioed by mass average, to obtain the solids as one layer from the saltcake and sludge layers

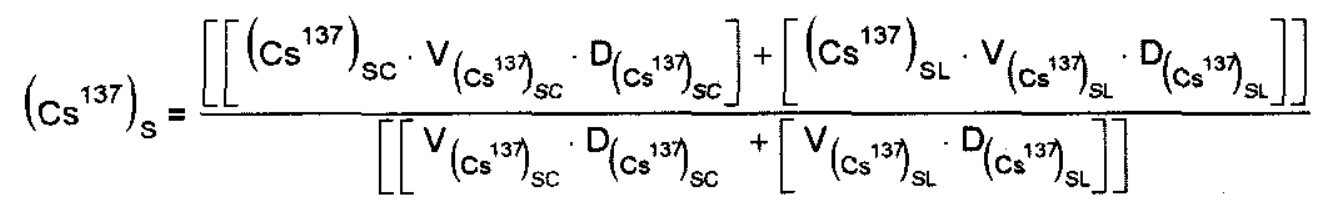

Equation 5

The weight percent $\mathrm{H}_{2} \mathrm{O}$ data from the TCD is reported in solid and liquids layers, so the solid layer was not combined at this point as with the other constituents. The total weight percent was calculated for each tank as a mass ratio using the waste volumes and densities:

$$
\mathrm{Wt} \% \%_{\mathrm{H} 2 \mathrm{O}_{\mathrm{T}}}=\frac{\left[\left(\mathrm{Wt} \% \mathrm{H}_{2} \mathrm{O}_{\mathrm{s}} \times \mathrm{V}_{\mathrm{H} 2 \mathrm{O}} \times \mathrm{D}_{\mathrm{H} 2 \mathrm{O}}\right)+\left(\mathrm{Wt} \% \mathrm{H} 2 \mathrm{O}_{\mathrm{L}} \times \mathrm{V}_{\mathrm{H} 2 \mathrm{O}} \cdot \mathrm{D}_{\mathrm{H} 2 \mathrm{O}}\right)\right]}{\left(\mathrm{V}_{\mathrm{H} 2 \mathrm{O}_{\mathrm{S}}} \cdot \mathrm{D}_{\mathrm{H} 2 \mathrm{O}}+\mathrm{V}_{\mathrm{H} 2 \mathrm{O}_{\mathrm{L}}} \cdot \mathrm{D}_{\mathrm{H} 2 \mathrm{O}}\right)}
$$

Equation 6

The new total concentration values from the liquid layer and combined solid layers, calculated with the distribution values, are then entered into Table 6 along with the weight percent $\mathrm{H}_{2} \mathrm{O}$ values. Only the liquid concentrations for the $\mathrm{Al}, \mathrm{NO}_{2}, \mathrm{NO}_{3}$ and $\mathrm{TOC}$ constituents are needed for the input to the Hydrogen Generation Rate Model, so the solid values are left blank. Also in Table 6, the tanks have been divided into SST and DST groupings. 
RPP - 6069 Rev. 0

This page intentionally left blank 


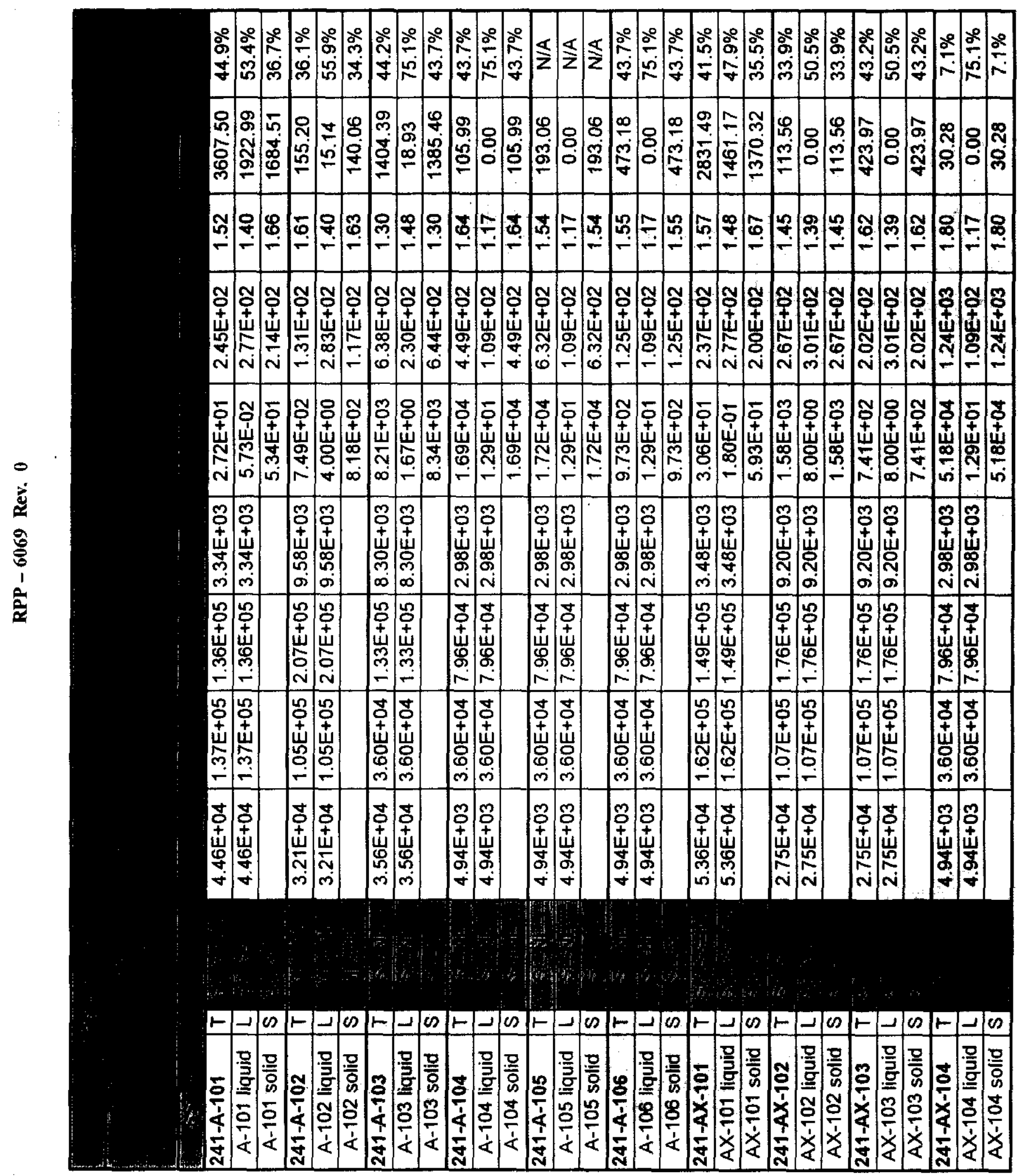




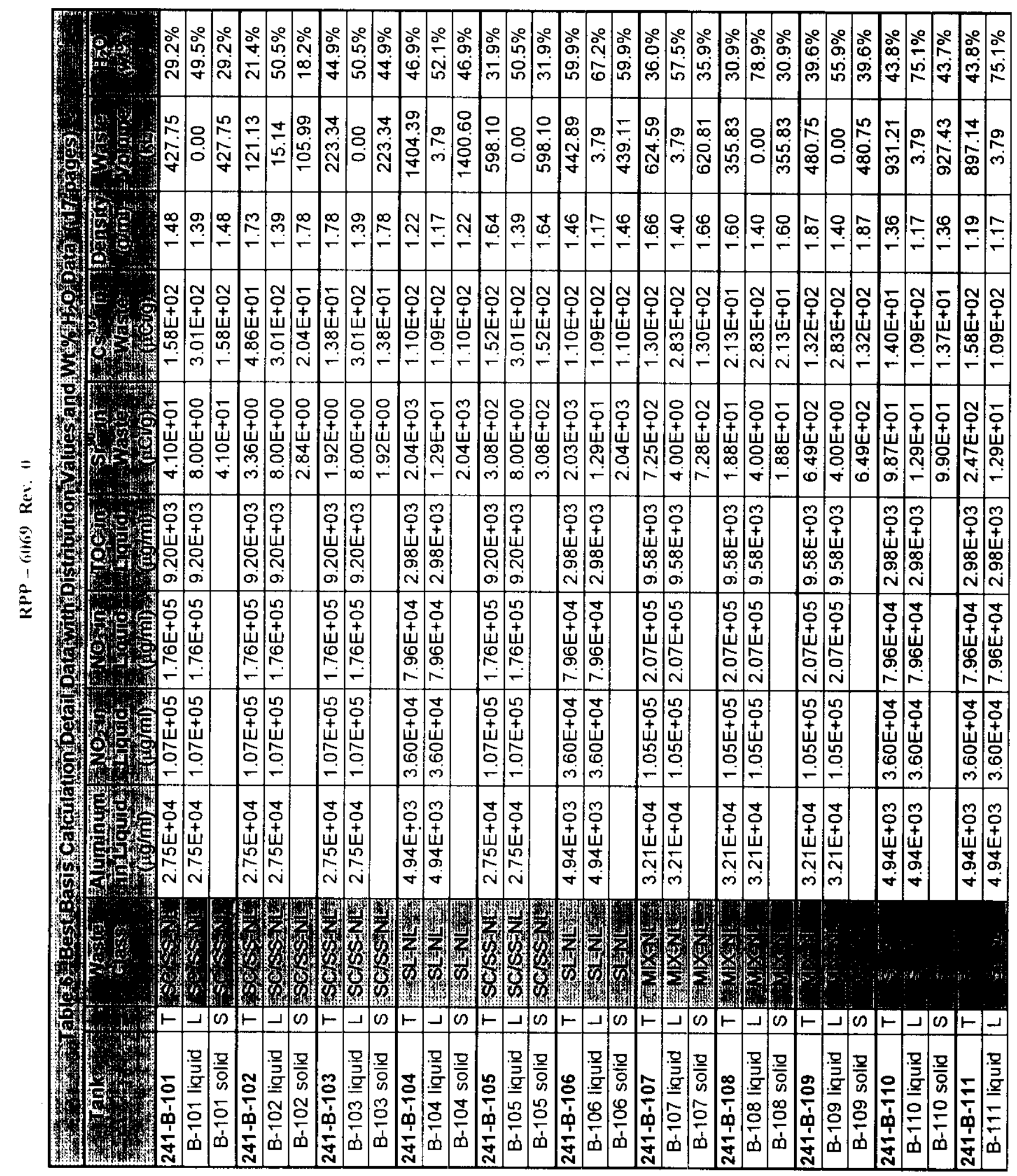




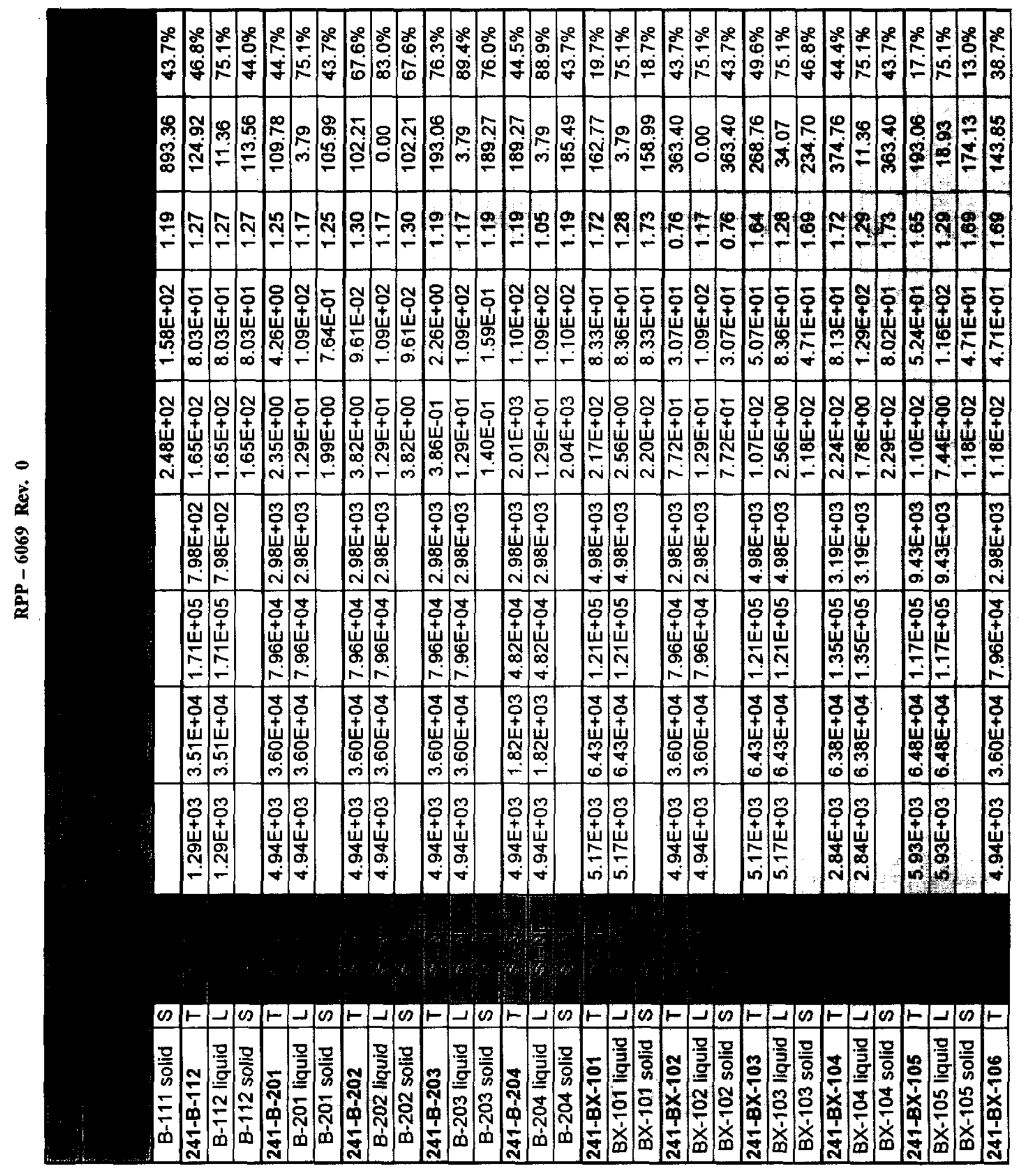




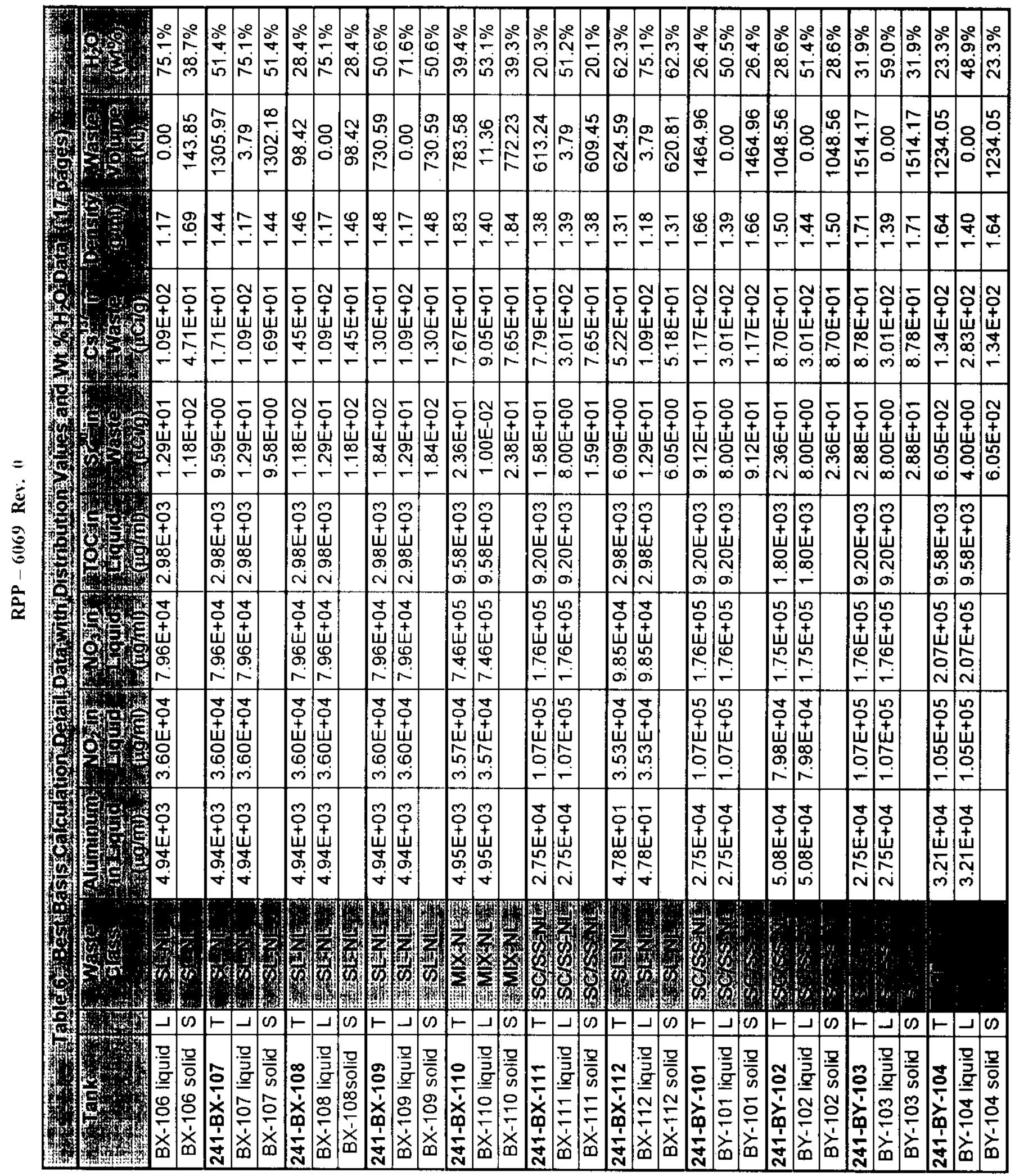




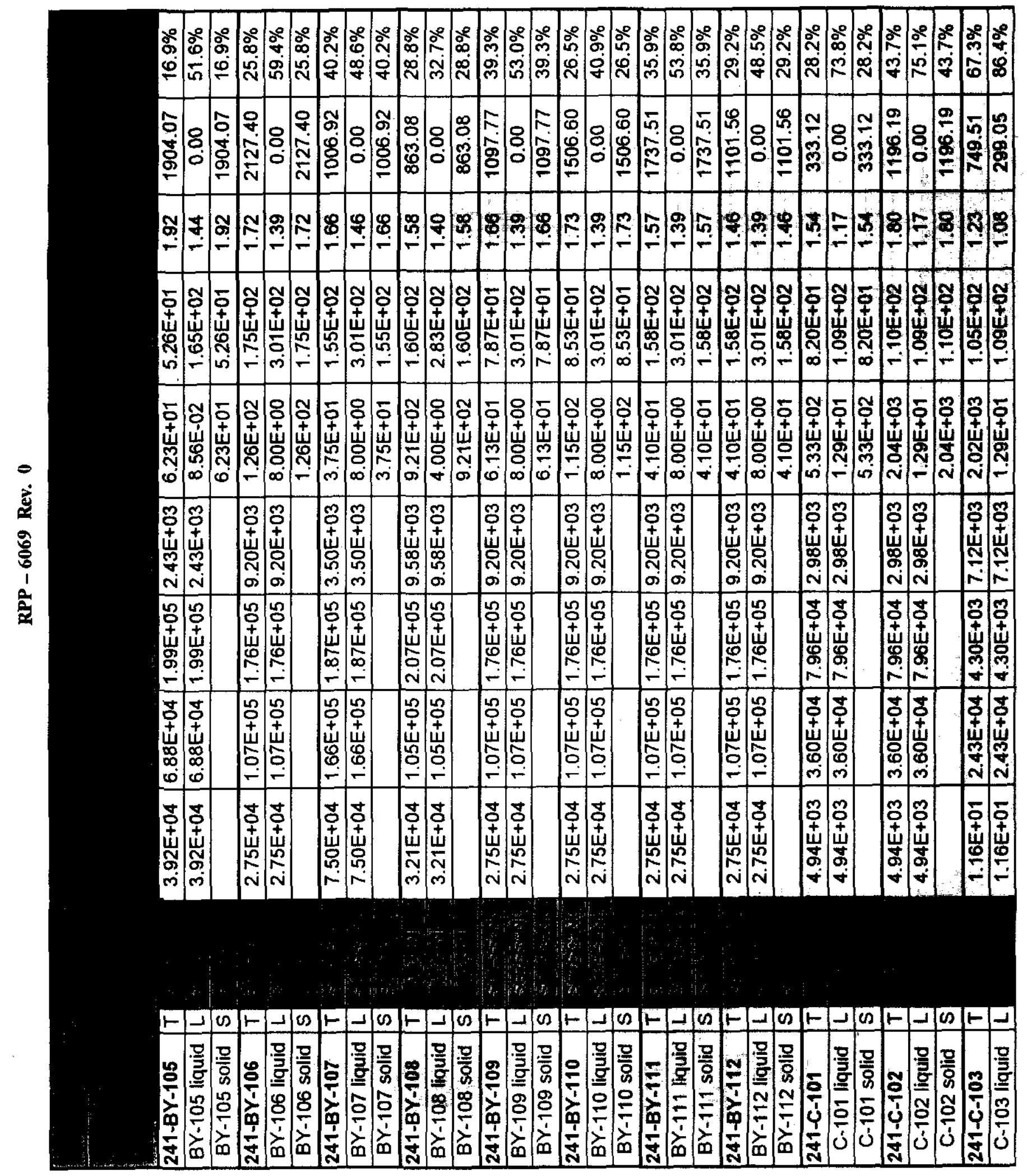




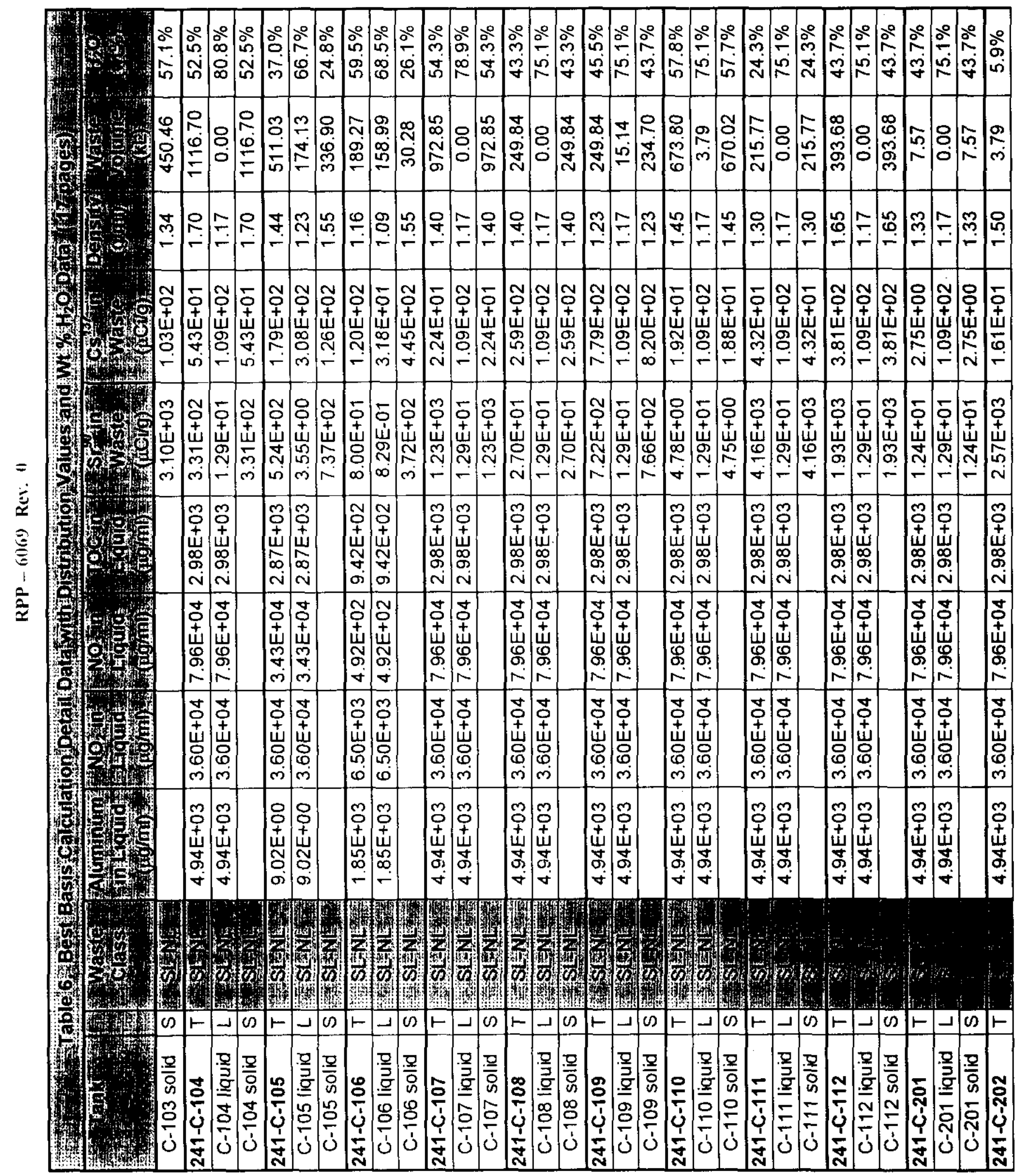




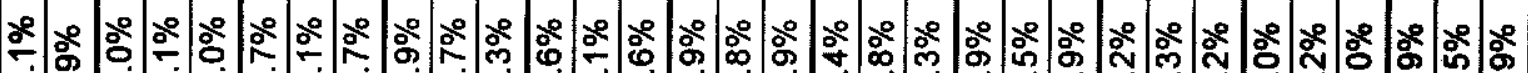

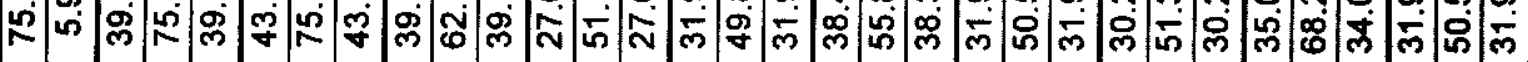

৪

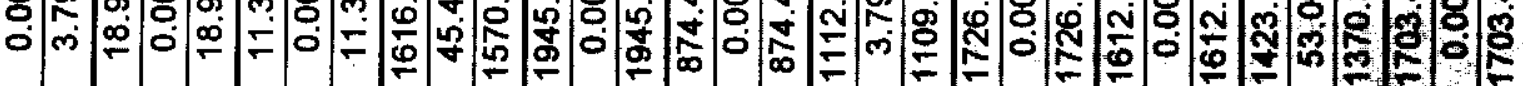

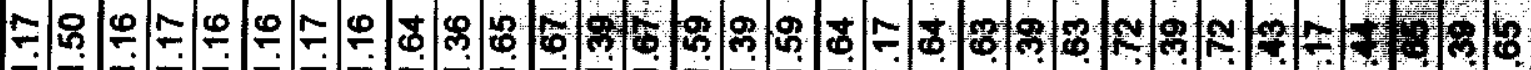

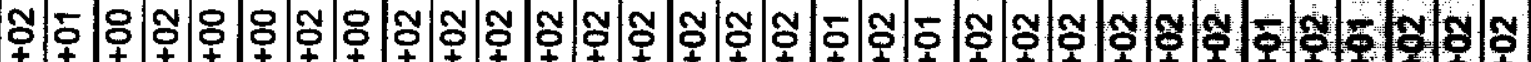

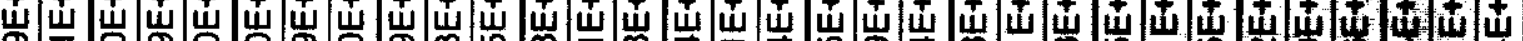

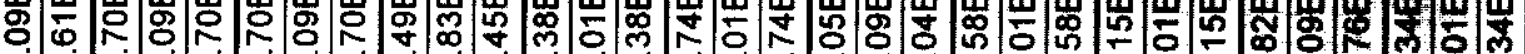

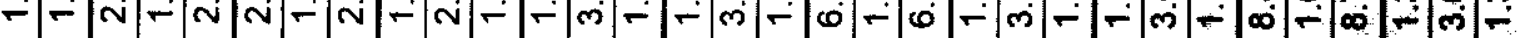

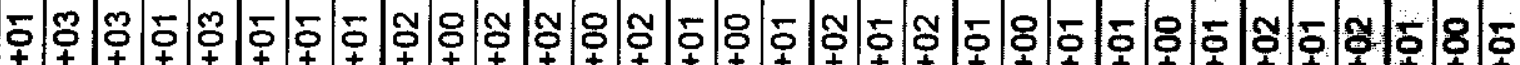

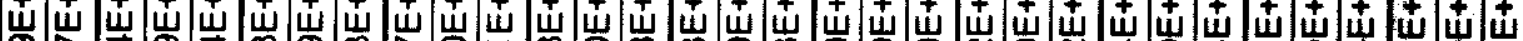
হ্స

\begin{tabular}{|c|c|c|c|c|c|c|c|c|c|c|c|c|c|c|c|c|c|c|c|c|c|c|}
\hline & $1 \circ$ & $-1 N$ & $-1-$ & - & -8 & + & $\mid \infty \infty^{\circ}$ & -2 & $\sim 1 \infty^{\circ}$ & i & $m$ & $m$ & $+\infty \infty^{\circ}$ & 0 & -1 & $\infty$ & $=17$ & $=$ & 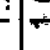 & N & 10 & \\
\hline & 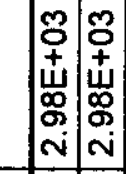 & & 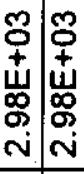 & & 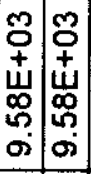 & & 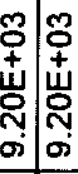 & & 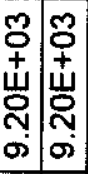 & & 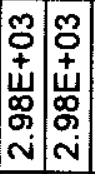 & & 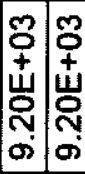 & & 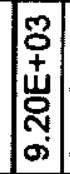 & 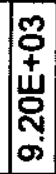 & & & & & 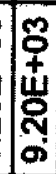 & \\
\hline & 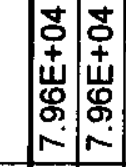 & & 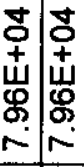 & & 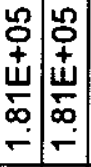 & & 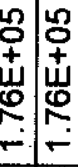 & & 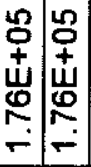 & & 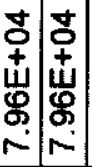 & & 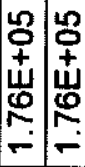 & & 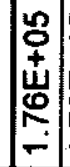 & 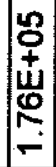 & & 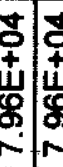 & & 骂 & 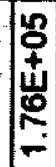 & \\
\hline & 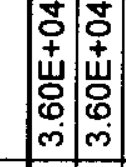 & & 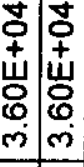 & & 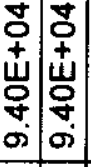 & & 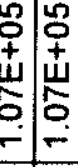 & & 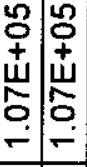 & & 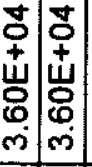 & & 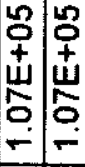 & 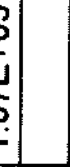 & $\mid \begin{array}{c}0 \\
0 \\
+ \\
w \\
0 \\
\vdots \\
-\end{array}$ & $\mid \begin{array}{c}0 \\
0 \\
+ \\
w \\
0 \\
0 \\
- \\
\end{array}$ & & 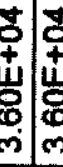 & & 足 & \begin{tabular}{l}
0 \\
+ \\
+ \\
$\mathbf{w}$ \\
$\vdots$ \\
\hdashline
\end{tabular} & \\
\hline & 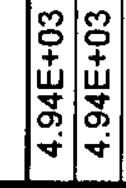 & & 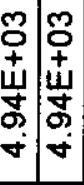 & & 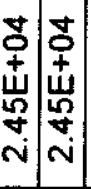 & & 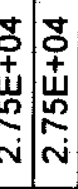 & & 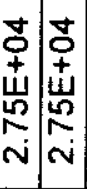 & & 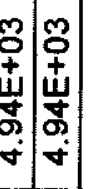 & & 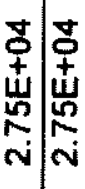 & & $\mid \begin{array}{c}\mathbf{J} \\
+ \\
w \\
\sim \\
N \\
v \\
v\end{array}$ & 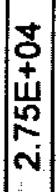 & & 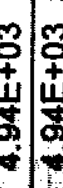 & & & 京 & \\
\hline
\end{tabular}

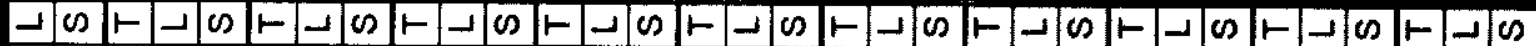

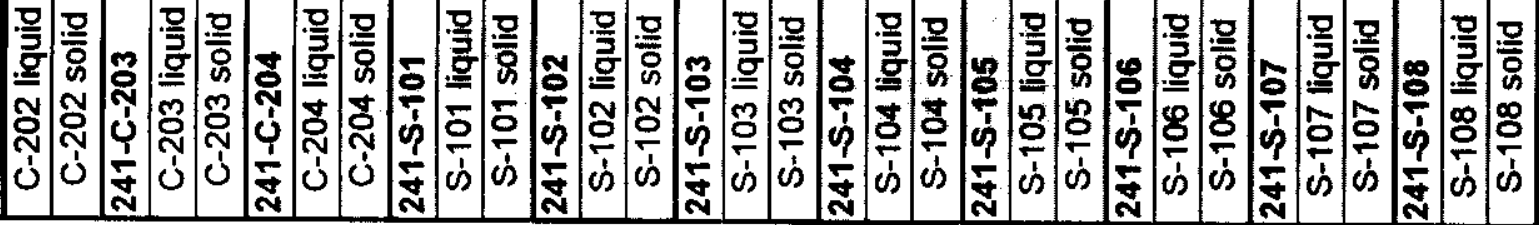




\begin{tabular}{|c|c|c|c|c|c|c|c|c|c|c|c|c|c|c|c|c|c|c|c|c|c|c|c|c|c|c|c|c|c|c|c|c|}
\hline m & $\mathrm{F}^{2}$ & $\begin{array}{l}0 \\
0 \\
0 \\
0\end{array}$ & 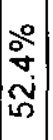 & $\mid \begin{array}{l}0 \\
0 \\
6 \\
6 \\
0\end{array}$ & $\mid$ & $\mid$ & 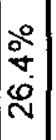 & 空 & $\left|\begin{array}{c}0 \\
0 \\
0 \\
m \\
0 \\
0\end{array}\right|$ & & $\mid$ & $\left|\begin{array}{c}0 \\
0 \\
0 \\
0 \\
0\end{array}\right|$ & 总 & 告 & 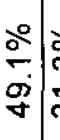 & 总 & 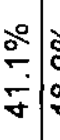 & . & 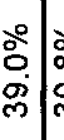 & 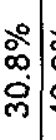 & 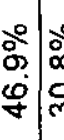 & . & & 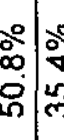 & $\begin{array}{c}\circ \\
\forall \\
\end{array}$ & . & & ?: & & & & \\
\hline a & 18 & $\mid \begin{array}{c}\bar{N} \\
\frac{\sigma}{\sigma} \\
\sigma\end{array}$ & 용 & $\mid$ & 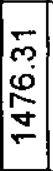 & $\begin{array}{l}8 \\
0 \\
0\end{array}$ & 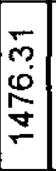 & $\left|\begin{array}{c}m \\
\dot{j} \\
\dot{c} \\
\text { v }\end{array}\right|$ & 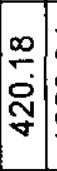 & 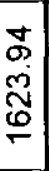 & $\mid \begin{array}{c}0 \\
\sigma \\
0 \\
\sigma\end{array}$ & $\begin{array}{l}8 \\
0 \\
0\end{array}$ & $\begin{array}{l}\Omega \\
0 \\
0 \\
0 \\
0 \\
T\end{array}$ & $\left|\begin{array}{c}0 \\
0 \\
0 \\
0 \\
0 \\
0\end{array}\right|$ & : & $\begin{array}{l}\infty \\
\infty \\
\dot{g} \\
\oplus \\
\emptyset\end{array}$ & 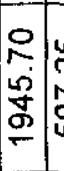 & 畓 & 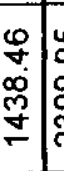 & 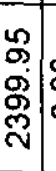 & o. & 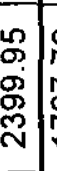 & & & 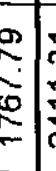 & 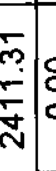 & & 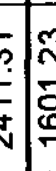 & & : & & \\
\hline as & 1 & $\bar{m}$ & $\stackrel{\text { m. }}{-}$ & $\stackrel{m}{m}$ & $\stackrel{0}{2}$ & 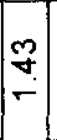 & $\stackrel{P}{i}$ & $\mid$ & 离 & 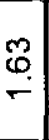 & 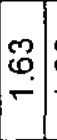 & $\stackrel{\mathscr{g}}{\stackrel{m}{-}}$ & $\begin{array}{c}m \\
0 \\
-\end{array}$ & 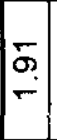 & $\left(\begin{array}{l}0 \\
- \\
-\end{array}\right.$ & $\bar{\sigma}$ & 电 & 告 & & $\begin{array}{l}\hat{n} \\
\therefore\end{array}$ & $\underset{f}{*}$ & 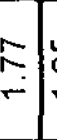 & 0 & $\begin{array}{l}\stackrel{m}{m} \\
\stackrel{5}{*}\end{array}$ & 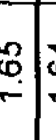 & $\begin{array}{l}0 \\
0 \\
-2\end{array}$ & 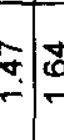 & ת & & $\stackrel{\varphi}{\circ}$ & $\left.\right|^{\infty}$ & 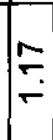 \\
\hline$\frac{8}{5}$ & & 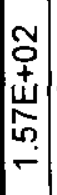 & \begin{tabular}{c}
\multirow{y}{0}{} \\
+ \\
$w$ \\
0 \\
0 \\
$m$
\end{tabular} & 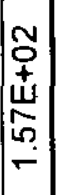 & $\mid \begin{array}{c}\overline{0} \\
+ \\
\omega \\
\bar{\sigma} \\
\sigma \\
\sigma\end{array}$ & 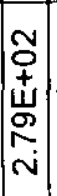 & 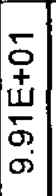 & 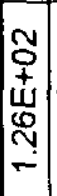 & 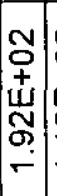 & 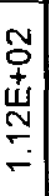 & 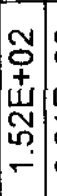 & 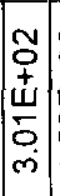 & 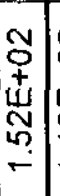 & 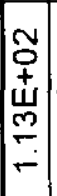 & $\left|\begin{array}{l}- \\
0 \\
+ \\
u \\
0 \\
⿱ 亠 䒑 \\
\end{array}\right|$ & 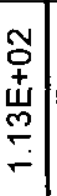 & 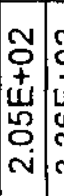 & 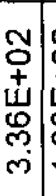 & 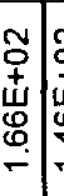 & 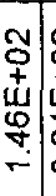 & 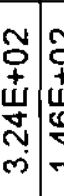 & 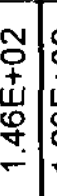 & & 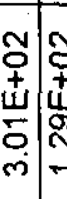 & 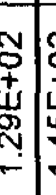 & 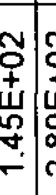 & 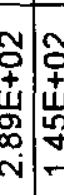 & 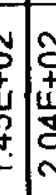 & & 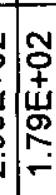 & & 岁 \\
\hline 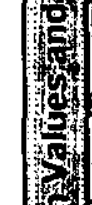 & & 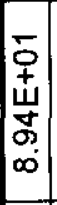 & $\mid \begin{array}{c}8 \\
8 \\
+ \\
4 \\
8 \\
0 \\
\infty\end{array}$ & 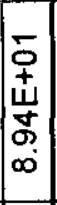 & 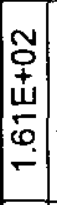 & 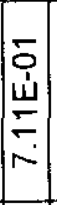 & 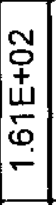 & 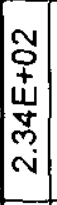 & 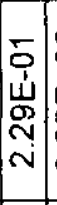 & 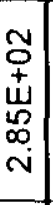 & 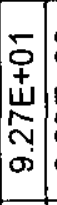 & $\left|\begin{array}{c}0 \\
0 \\
4 \\
4 \\
0 \\
\infty \\
\infty\end{array}\right|$ & 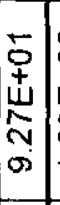 & 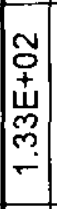 & 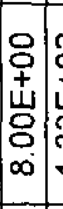 & 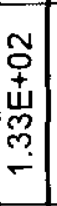 & 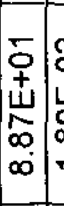 & 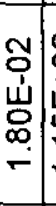 & 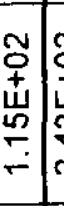 & 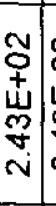 & 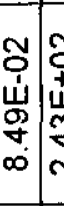 & 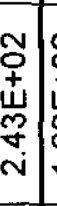 & 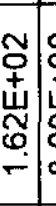 & 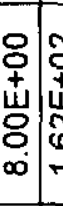 & 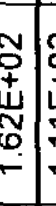 & 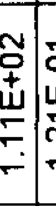 & 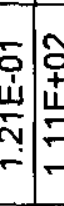 & 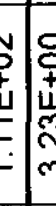 & & $\begin{array}{l}8 \\
0 \\
4 \\
\\
\\
\end{array}$ & & $\overrightarrow{\mathfrak{N}}$ \\
\hline 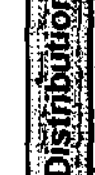 & 3 & $\begin{array}{c}m \\
0 \\
+ \\
0 \\
0 \\
0 \\
0\end{array}$ & 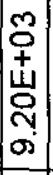 & & $\mid \begin{array}{c}m \\
0 \\
+ \\
m \\
0 \\
0 \\
0 \\
\end{array}$ & \begin{tabular}{|c|} 
\\
0 \\
+ \\
$⿱ 山$ \\
0 \\
0 \\
0 \\
\end{tabular} & & $\mid \begin{array}{c}m \\
0 \\
+ \\
\infty \\
\infty \\
m \\
-2 \\
-1\end{array}$ & \begin{tabular}{|c|}
$m$ \\
0 \\
+ \\
$w$ \\
$\infty$ \\
$m$ \\
-2 \\
\end{tabular} & & 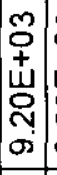 & $\mid \begin{array}{c}m \\
0 \\
+ \\
w \\
0 \\
0 \\
\sigma\end{array}$ & & $\mid \begin{array}{c}N \\
0 \\
+ \\
w \\
w \\
m \\
m\end{array}$ & $\begin{array}{c}N \\
0 \\
+ \\
w \\
w \\
m \\
m \\
\end{array}$ & & 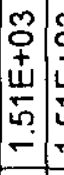 & 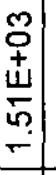 & & 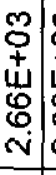 & 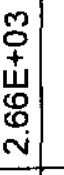 & & & 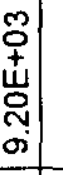 & & 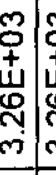 & 岕 & 5 & & & & in \\
\hline 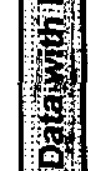 & o & $\begin{array}{c}n \\
0 \\
+ \\
0 \\
0 \\
-2 \\
-2 \\
\end{array}$ & $\begin{array}{c}n \\
0 \\
+ \\
w \\
0 \\
2 \\
-\end{array}$ & & 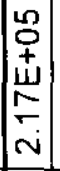 & 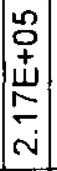 & & 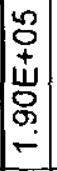 & 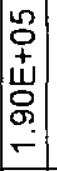 & & 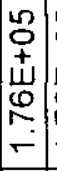 & $\mid \begin{array}{c}0 \\
0 \\
+ \\
4 \\
0 \\
? \\
- \\
-\end{array}$ & & $\mid \begin{array}{c}n \\
0 \\
+ \\
4 \\
0 \\
0 \\
- \\
\end{array}$ & $\mid \begin{array}{c}0 \\
0 \\
+ \\
w \\
0 \\
0 \\
-1\end{array}$ & & 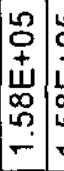 & 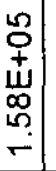 & & $\begin{array}{l}0 \\
0 \\
+ \\
5 \\
0 \\
-\end{array}$ & . & & & . & & צ's & . & $w$ & $4 \pi$ & & & \\
\hline क & $\begin{array}{l}3 \\
0 \\
0\end{array}$ & 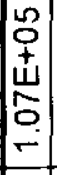 & $\mid \begin{array}{c}0 \\
0 \\
+ \\
w \\
0 \\
0 \\
-2 \\
-\end{array}$ & & 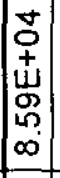 & $\mid \begin{array}{c}\forall \\
0 \\
\pm \\
⿱ 亠 䒑 \\
0 \\
\infty \\
\infty \\
\end{array}$ & & 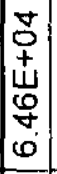 & 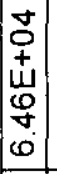 & & $\begin{array}{c}0 \\
0 \\
+ \\
w \\
\vdots \\
0 \\
-\end{array}$ & 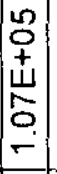 & & 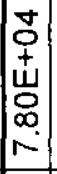 & 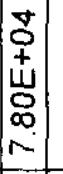 & & 的 & 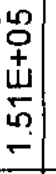 & & $\begin{array}{c}w \\
0 \\
+ \\
w \\
0 \\
0 \\
- \\
-\end{array}$ & $\begin{array}{l}0 \\
0 \\
+ \\
+ \\
\infty\end{array}$ & & & \begin{tabular}{l}
+ \\
\multirow{4}{*}{} \\
0 \\
- \\
\end{tabular} & & 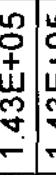 & & 告 & & & & \\
\hline 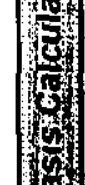 & $\begin{array}{l}5 \\
5 \\
5\end{array}$ & 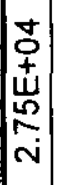 & & & 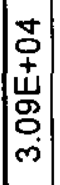 & $\left|\begin{array}{c}0 \\
0 \\
\vdots \\
w \\
0 \\
0 \\
\dot{m}\end{array}\right|$ & & 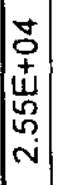 & $\begin{array}{c} \pm \\
0 \\
+ \\
w \\
\sim \\
\sim \\
\sim \\
\sim\end{array}$ & & $\begin{array}{c} \pm \\
0 \\
+ \\
w \\
\tilde{N} \\
\sim \\
\sim\end{array}$ & 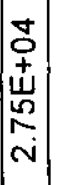 & & 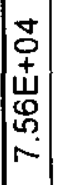 & $\left|\begin{array}{l}8 \\
0 \\
+ \\
u \\
0 \\
0 \\
-2\end{array}\right|$ & & 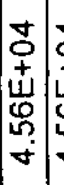 & \begin{tabular}{|c|}
+ \\
4 \\
0 \\
0 \\
$\dot{0}$
\end{tabular} & & $\begin{array}{l}0 \\
+ \\
\square \\
\dot{+} \\
\dot{v}\end{array}$ & & & & & & 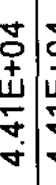 & 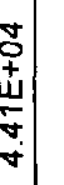 & & & & & \\
\hline 4 & $\frac{6}{-3}$ & $\begin{array}{l}x \\
8 \\
8\end{array}$ & : & & 3 & a & in & 6 & 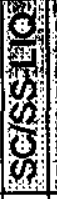 & $w_{5}$ & 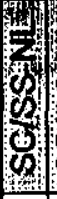 & 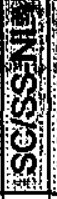 & a & 6 & 6 & 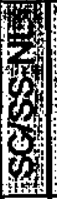 & 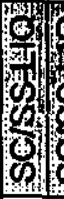 & 8 & 皿 & 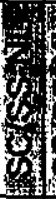 & x) & 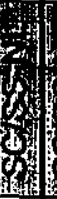 & 28: & 4 & & in & & & & & & \\
\hline 5 & $\because 4$ & $\vdash$ & \lrcorner & $\infty$ & 1 & $\Delta$ & $\infty$ & 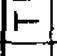 & -1 & 0 & 1 & $\Delta$ & (o) & 1 & -10 & $\infty$ & $1-$ & $\triangle$ & $\infty$ & $\vdash$ & $\Delta \mathrm{C}$ & o & $\vdash$ & $\Delta$, & 0 & 1 & $=0$ & 01 & & 5 & $\vdash$ & -1 \\
\hline 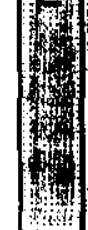 & 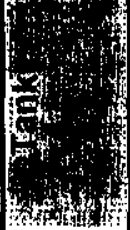 & $\frac{\pi}{0}$ & 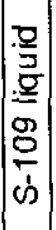 & $\mid \begin{array}{l}\text { 몽 } \\
0 \\
4 \\
\text { g } \\
\\
1\end{array}$ & 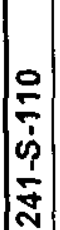 & $\mid \begin{array}{l}\frac{9}{2} \\
\frac{\bar{z}}{0} \\
\frac{0}{\dot{s}} \\
\end{array}$ & $\begin{array}{l}\frac{0}{0} \\
0 \\
0 \\
\frac{0}{6} \\
\dot{\omega}\end{array}$ & 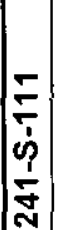 & $\left|\begin{array}{c}\frac{q}{3} \\
\underline{\underline{\sigma}} \\
\bar{\sigma} \\
\bar{\omega}\end{array}\right|$ & w & 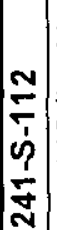 & $\left|\begin{array}{c}\frac{g}{3} \\
\frac{\sigma}{\sigma} \\
\frac{a}{\sigma} \\
\bar{c}\end{array}\right|$ & 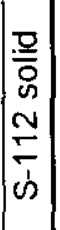 & 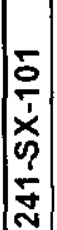 & 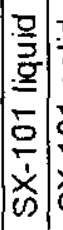 & 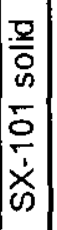 & 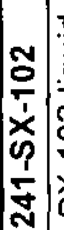 & 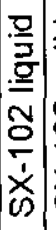 & 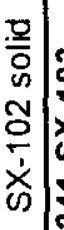 & 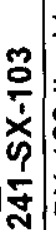 & 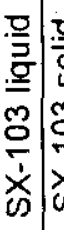 & $\begin{array}{l}\frac{9}{0} \\
0 \\
6 \\
3 \\
0 \\
\dot{1} \\
\dot{1} \\
0\end{array}$ & 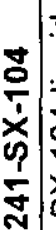 & 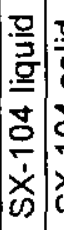 & n & 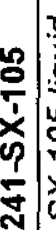 & 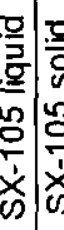 & 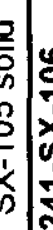 & 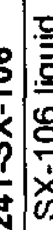 & 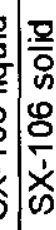 & $\frac{\sqrt{4}}{\frac{\pi}{4}}$ & $\frac{1}{1}$ \\
\hline
\end{tabular}




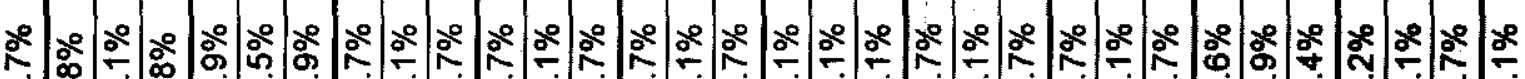
m

: ల్ల

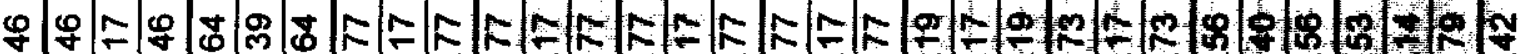

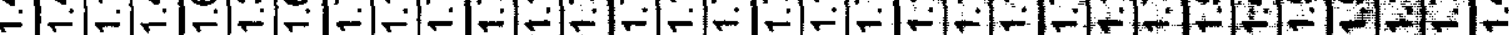

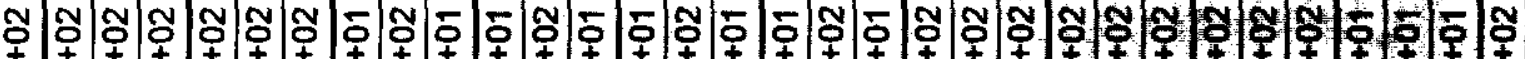

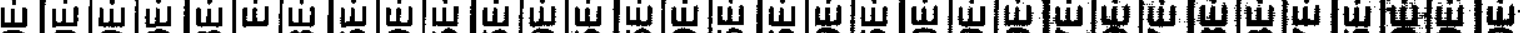

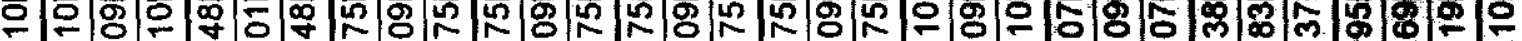

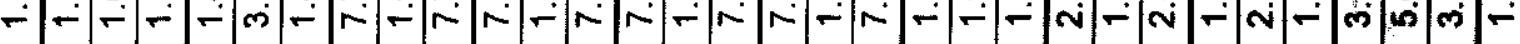

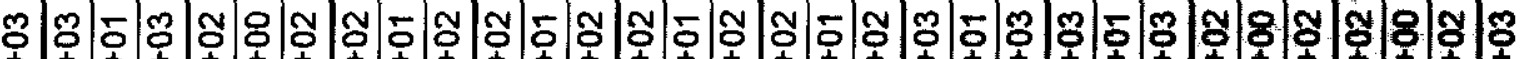

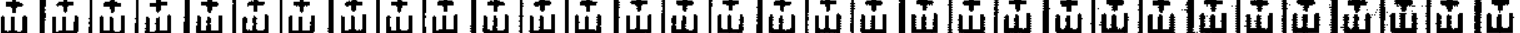

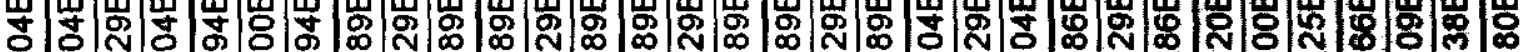

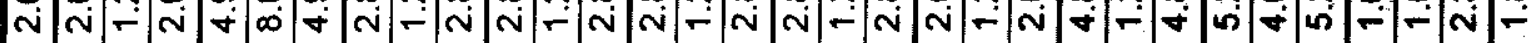

\begin{tabular}{|c|c|c|c|c|c|c|c|c|c|c|c|c|c|c|c|c|c|c|c|c|}
\hline 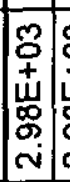 & 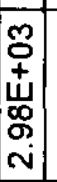 & 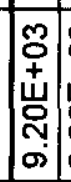 & 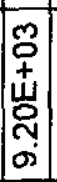 & 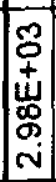 & $\begin{array}{c}- \\
0 \\
+ \\
w \\
\infty \\
o \\
\sim \\
\sim\end{array}$ & 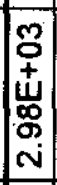 & 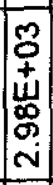 & 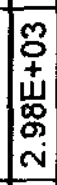 & $\begin{array}{l}m \\
0 \\
+ \\
\dot{w} \\
\infty \\
\sigma \\
\sim \\
\sim \\
\end{array}$ & 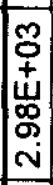 & 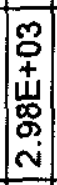 & & 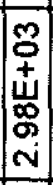 & 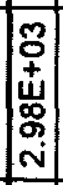 & 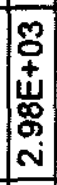 & & 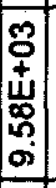 & 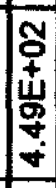 & 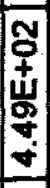 & 18 \\
\hline 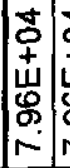 & 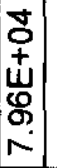 & 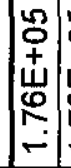 & $\mid \begin{array}{c}n \\
0 \\
+ \\
w \\
0 \\
2 \\
-2\end{array}$ & 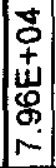 & 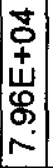 & 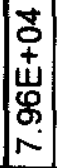 & 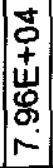 & 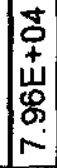 & 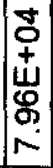 & 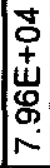 & 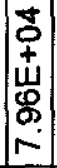 & 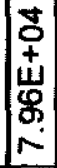 & 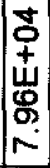 & 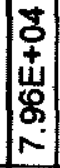 & 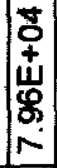 & 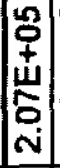 & 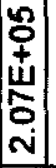 & 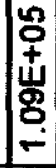 & 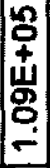 & \\
\hline $\begin{array}{l}8 \\
0 \\
+ \\
0 \\
0 \\
0 \\
m\end{array}$ & $\begin{array}{l} \pm \\
+ \\
+ \\
\dot{0} \\
0 \\
\dot{m}\end{array}$ & {$\left[\begin{array}{c}n \\
0 \\
+ \\
w \\
0 \\
0 \\
-\end{array}\right.$} & 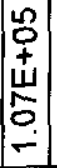 & 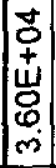 & 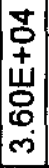 & 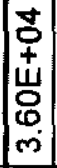 & 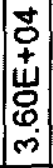 & 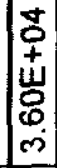 & 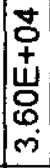 & 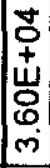 & 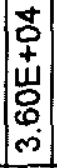 & $\mid \begin{array}{l}\mathbf{y} \\
0 \\
+ \\
\text { 岁 } \\
0 \\
\dot{m}\end{array}$ & 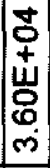 & 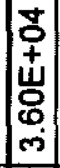 & 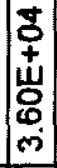 & $\mid \begin{array}{c}0 \\
0 \\
+ \\
⿱ 屮 凵 \\
0 \\
0 \\
-\end{array}$ & 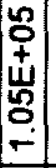 & 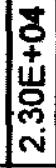 & 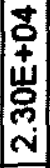 & \\
\hline $\mid \begin{array}{c}\mathscr{8} \\
\dot{+} \\
\dot{W} \\
\dot{\sigma} \\
\dot{+}\end{array}$ & 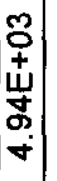 & $\mid \begin{array}{c}\mathbf{0} \\
+ \\
w \\
\tilde{W} \\
\sim \\
\sim\end{array}$ & 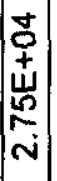 & 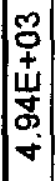 & 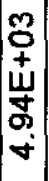 & 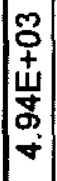 & 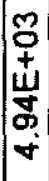 & 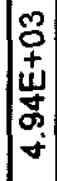 & $\begin{array}{l}\mathscr{O} \\
0 \\
\dot{w} \\
\forall\end{array}$ & & 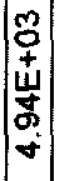 & 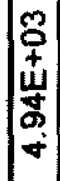 & 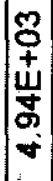 & 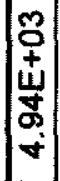 & 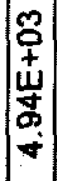 & 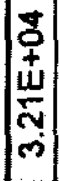 & 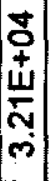 & 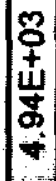 & 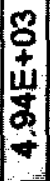 & \\
\hline
\end{tabular}

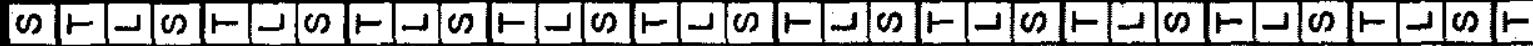

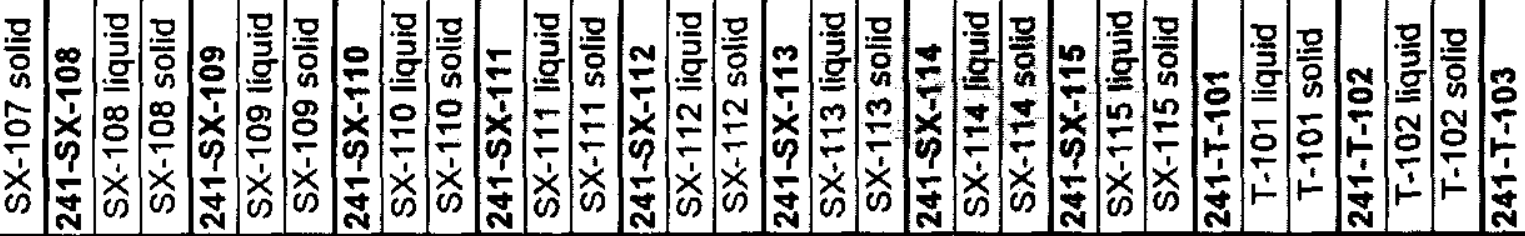




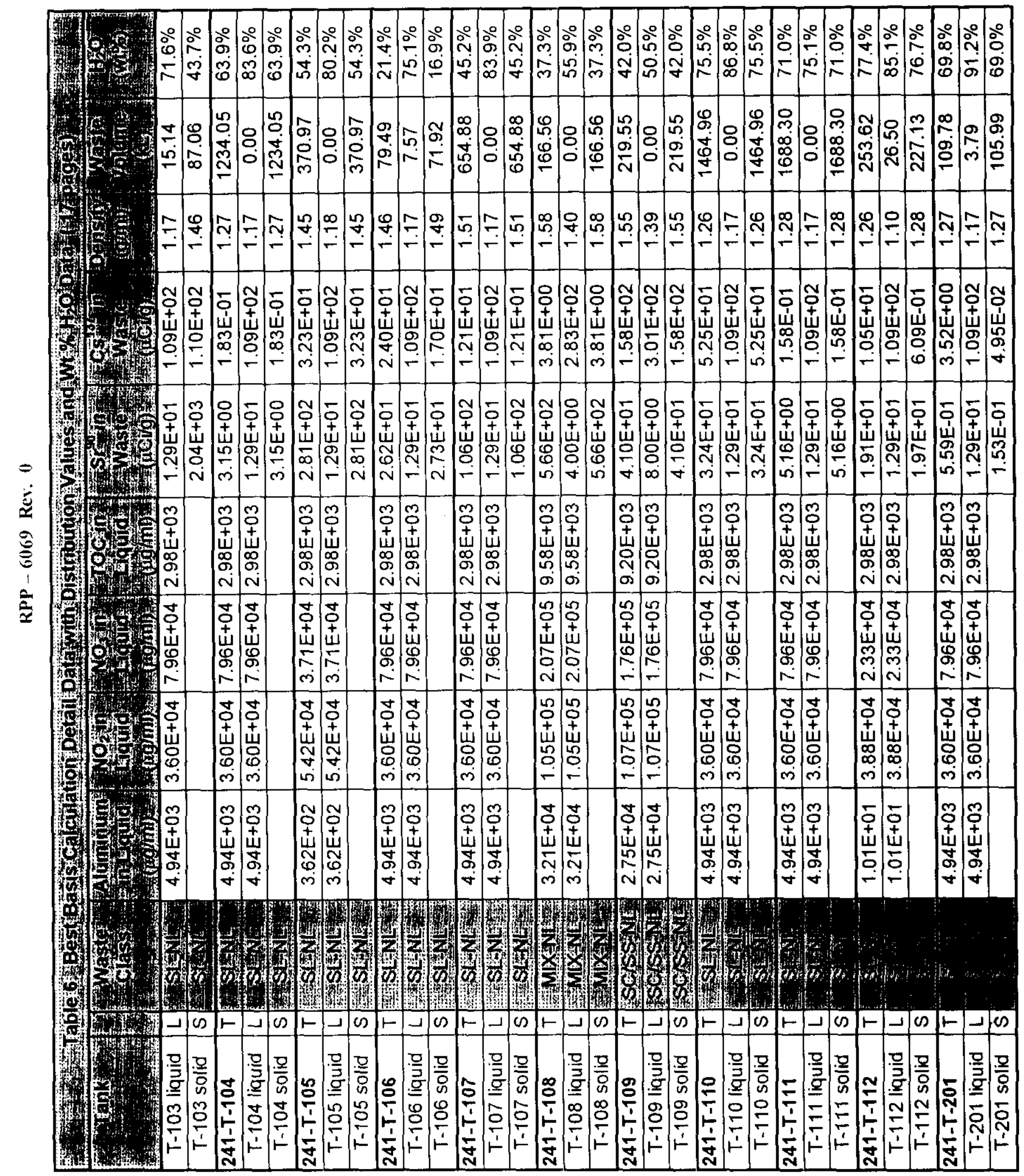




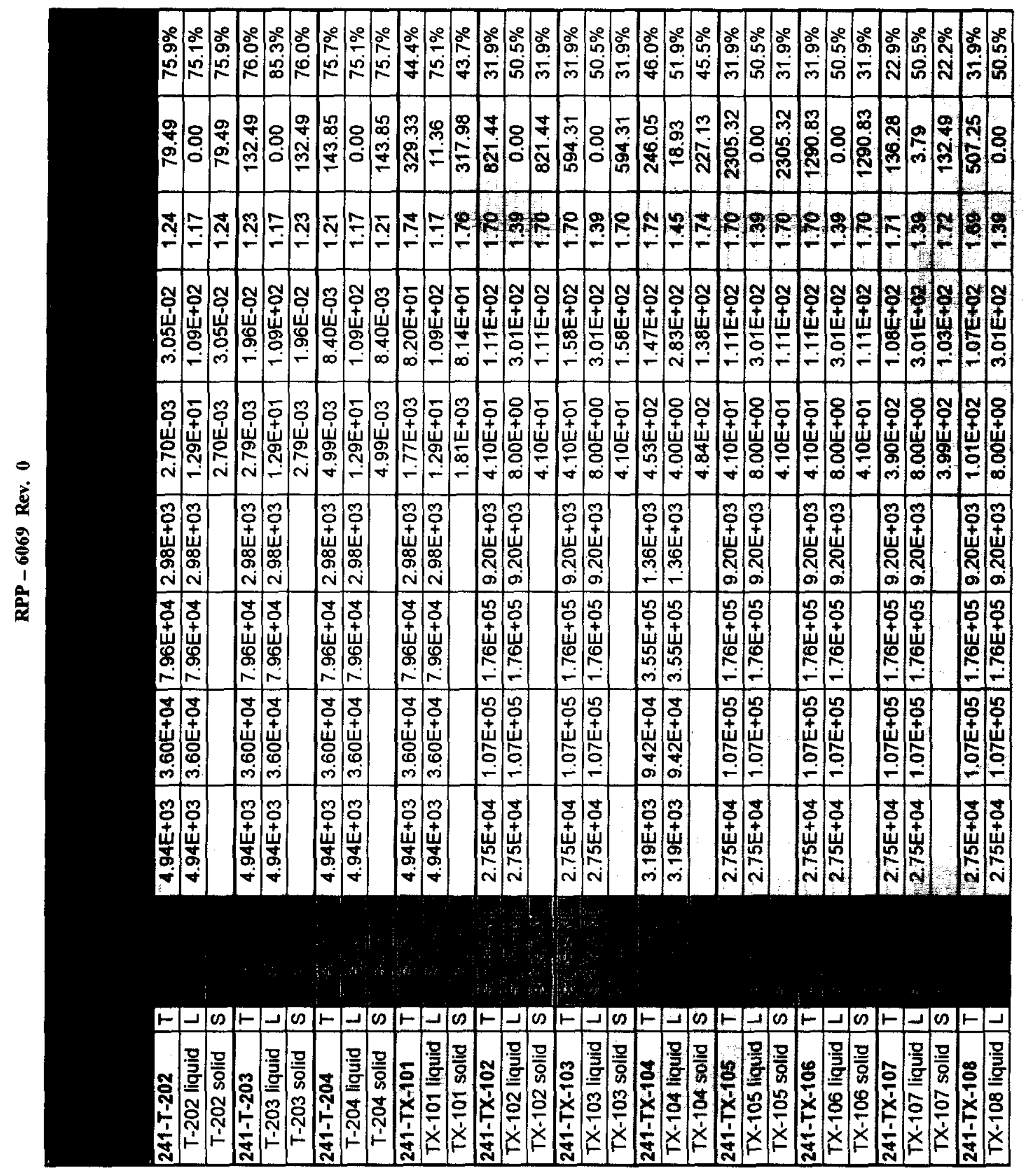




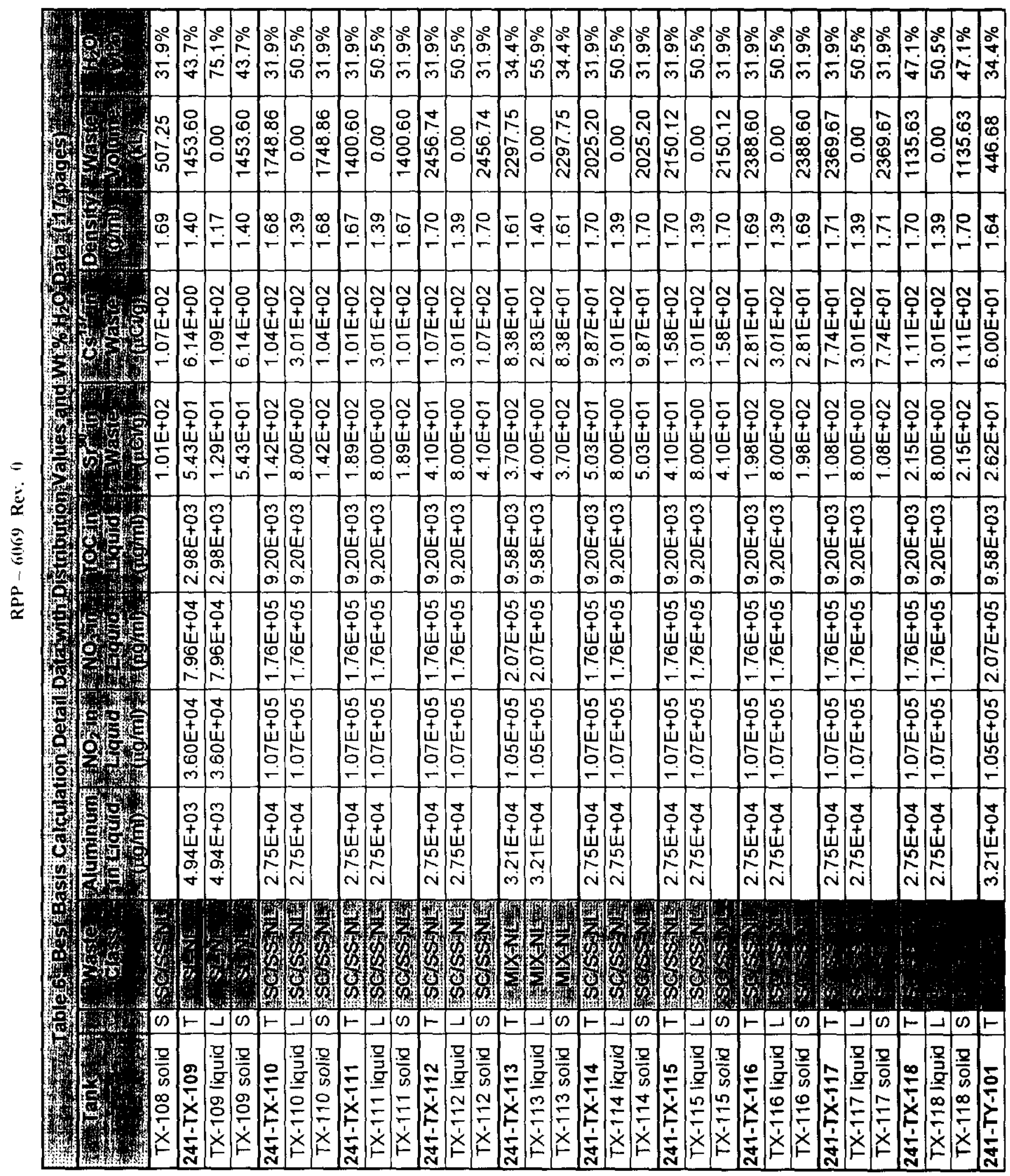




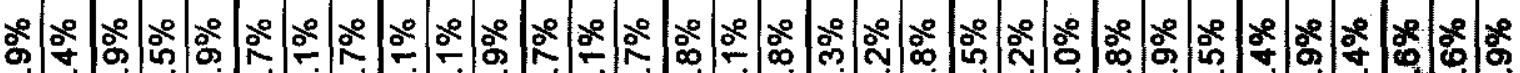

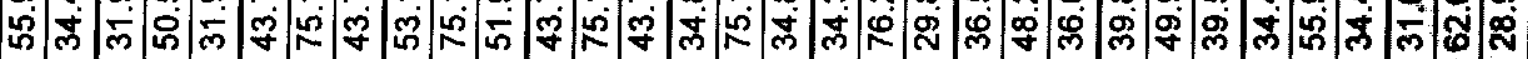

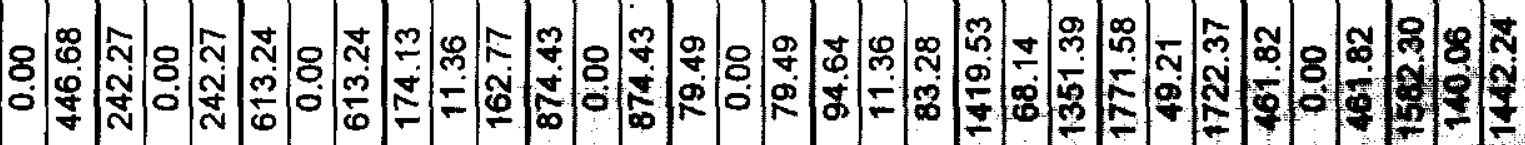

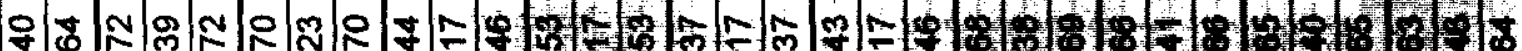

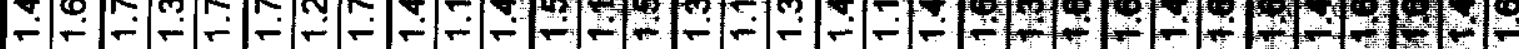

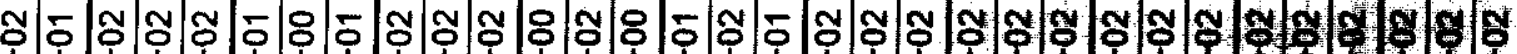

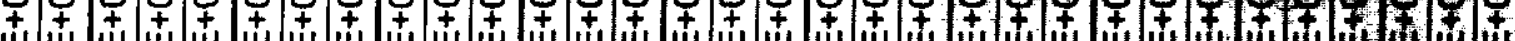

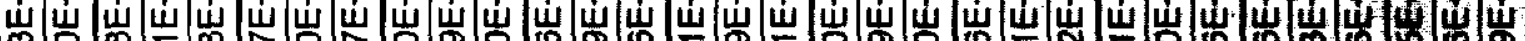

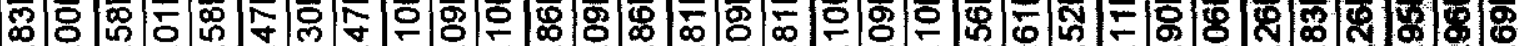

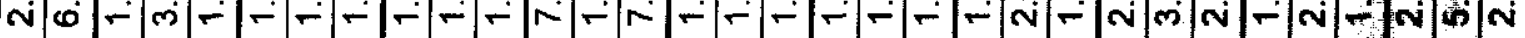

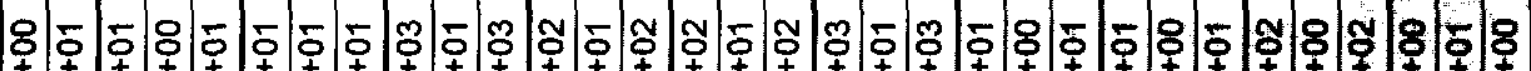

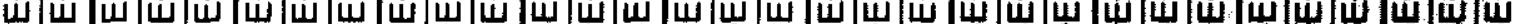

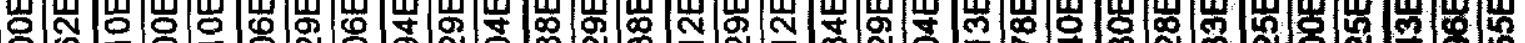

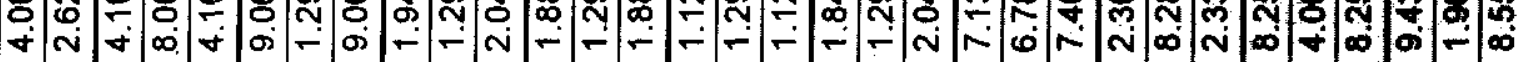

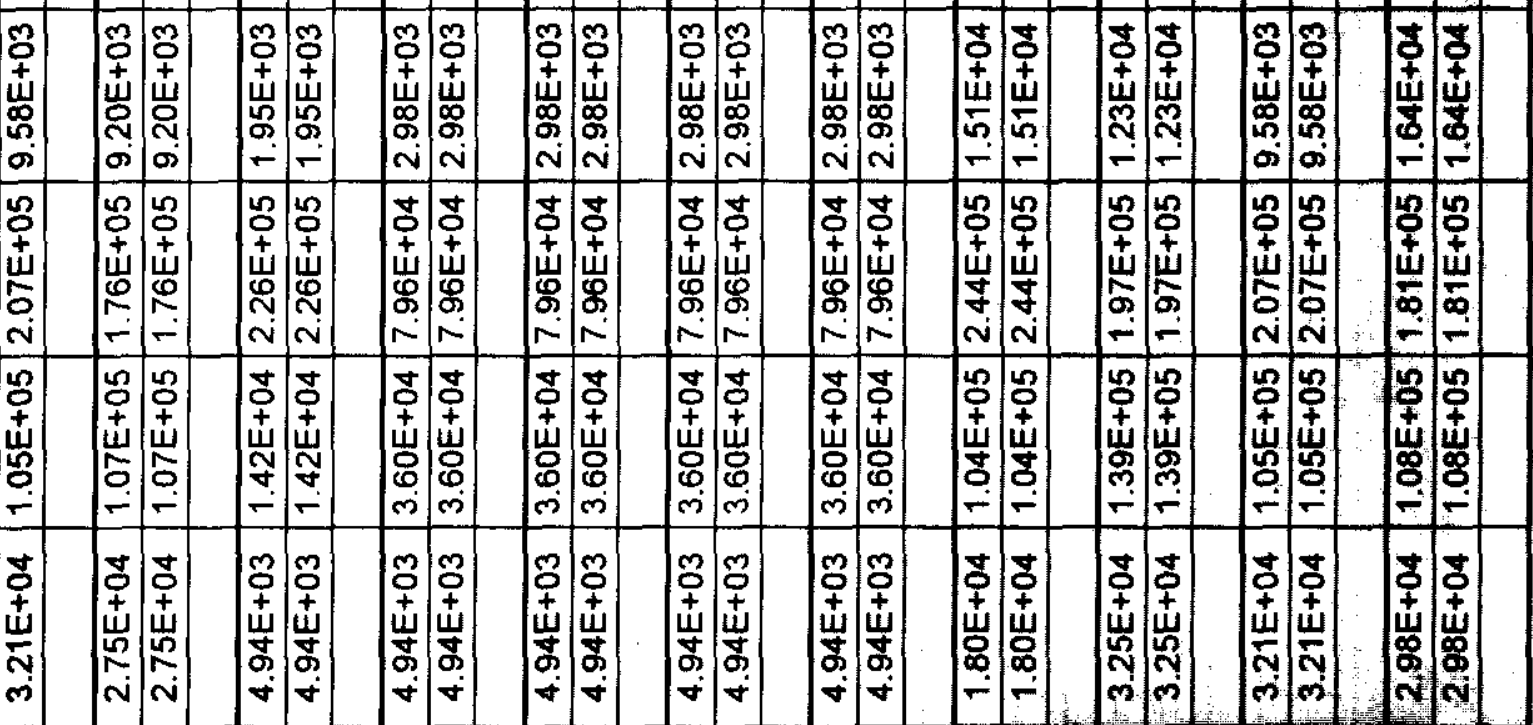

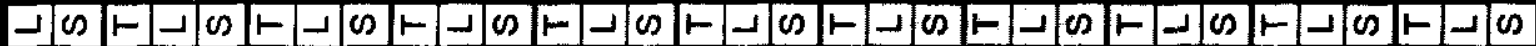
을

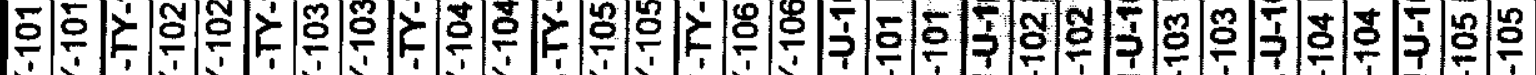

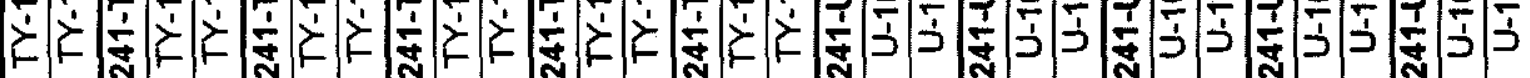




\begin{tabular}{|c|c|c|c|c|c|c|c|c|c|c|c|c|c|c|c|c|c|c|c|c|c|c|c|c|c|c|c|c|c|c|c|}
\hline 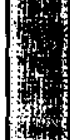 & $\frac{x}{x+2}$ & 票 & $\mid$ & , & $\mid$ & $\begin{array}{l}0 \\
\infty \\
\infty \\
9 \\
9\end{array}$ & & . & $\mid$ & & 总 & & $\begin{array}{l}\stackrel{\circ}{\circ} \\
\stackrel{2}{N}\end{array}$ & & 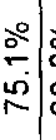 & 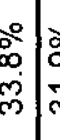 & $\begin{array}{l}\circ \\
\vdots \\
\dot{m}\end{array}$ & 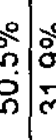 & 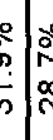 & $\frac{5}{5}$ & $\begin{array}{l}0 \\
0 \\
i \\
0\end{array}$ & & $\vdots$ & 交 & 今̊ & 永 & $\mid \begin{array}{c}0 \\
0 \\
0 \\
\text { N }\end{array}$ & 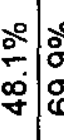 & ( & & $\mid \begin{array}{l}2 \\
2 \\
0 \\
0\end{array}$ \\
\hline mot & 8 & $\mid$ & $\left|\begin{array}{c}\infty \\
0 \\
0 \\
1\end{array}\right|$ & 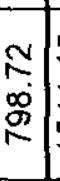 & 番 & 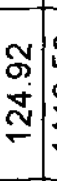 & | & 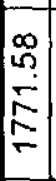 & $\mid$ & $\begin{array}{l}m \\
\stackrel{n}{0} \\
0 \\
0 \\
-\end{array}$ & $\begin{array}{c}N \\
0 \\
0 \\
\infty \\
\end{array}$ & \begin{tabular}{l}
$\mathcal{N}$ \\
$\sigma$ \\
\hdashline \\
$\bar{T}$
\end{tabular} & $\begin{array}{l}0 \\
0 \\
\infty \\
0 \\
0 \\
0\end{array}$ & $\begin{array}{l}8 \\
8 \\
\dot{0} \\
\text { d }\end{array}$ & 8 & $\begin{array}{lll}8 \\
8 \\
\dot{0}\end{array}$ & 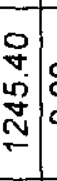 & $\int_{8}^{\frac{7}{2}}$ & 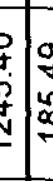 & & : & & & $\frac{1}{2}$ & $\left|\begin{array}{l}3 \\
0 \\
\infty \\
0\end{array}\right|$ & $\begin{array}{c}q \\
\dot{m}\end{array} \mid$ & $\frac{\pi}{m}$ & "lo & \begin{tabular}{l|l}
9 & $n$ \\
$m$ & $n$
\end{tabular} & $\underset{m}{\mathscr{D}}$ & \\
\hline s & & $\stackrel{8}{\varnothing}$ & m & ợ & $\mid$ & $\underset{\square}{\sigma}$ & $?$ & $\mid \begin{array}{c}\mathbb{N} \\
-2\end{array}$ & 悐 & 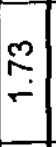 & $\stackrel{\mathscr{g}}{-}$ & 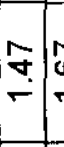 & $\stackrel{-}{\circ}$ & $=$ & $\underset{5}{5}$ & $\begin{array}{l}\mathscr{v} \\
- \\
\end{array}$ & ְ. & & كَّ & 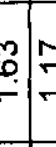 & $=$ & & $\stackrel{F}{F}$ & $\stackrel{\widetilde{\sigma}}{\sigma}$ & $\mid \begin{array}{l}m \\
\sim \\
\sim\end{array}$ & 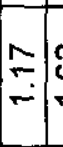 & $\left(\begin{array}{c}0 \\
\stackrel{0}{0}\end{array}\right.$ & 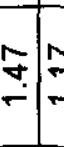 & $\because \underset{\sigma}{F}$ & $\stackrel{\mathscr{N}}{\stackrel{2}{*}}$ & $=$ \\
\hline 政 & 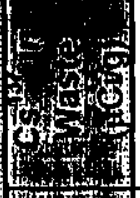 & 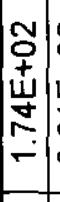 & 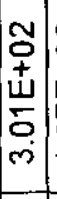 & $\begin{array}{c}\mathcal{N} \\
+ \\
w \\
w \\
o \\
-\end{array}$ & $\begin{array}{c}5 \\
\vdots \\
4 \\
m \\
0 \\
0 \\
0\end{array}$ & 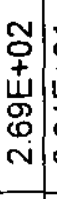 & & 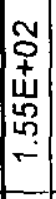 & 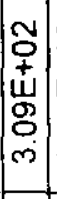 & 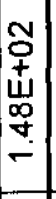 & $\begin{array}{c}N \\
0 \\
\pm \\
w \\
w \\
-2\end{array}$ & 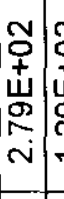 & 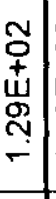 & 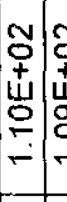 & 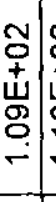 & 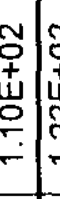 & 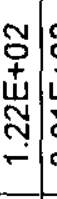 & 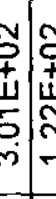 & 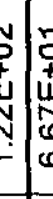 & 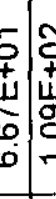 & 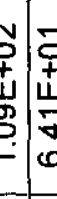 & $\mid \begin{array}{l}2 \\
0 \\
1 \\
5 \\
5 \\
5\end{array}$ & : & 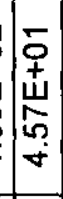 & $\left|\begin{array}{c}5 \\
+ \\
w \\
\vdots \\
\omega \\
0\end{array}\right|$ & $\begin{array}{c}0 \\
0 \\
+ \\
w \\
0 \\
0 \\
-2\end{array}$ & 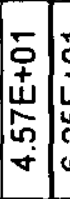 & 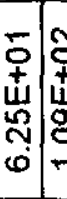 & 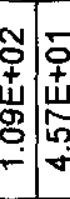 & & 要 \\
\hline 数 & $\left(\begin{array}{c}6 \\
6\end{array}\right.$ & 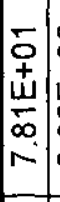 & $\left|\begin{array}{c}8 \\
+ \\
\pm \\
0 \\
0 \\
\infty \\
\infty\end{array}\right|$ & 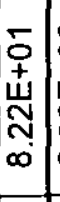 & 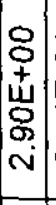 & 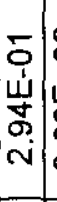 & 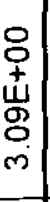 & 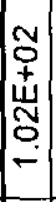 & $\left|\begin{array}{c}0 \\
0 \\
+ \\
w \\
8 \\
\infty \\
\infty\end{array}\right|$ & 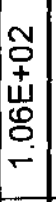 & 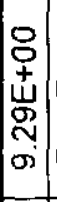 & 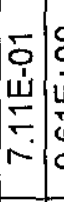 & $\begin{array}{c}8 \\
+ \\
\pm \\
\\
\vdots \\
0\end{array}$ & 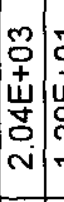 & 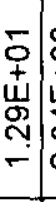 & 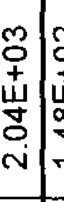 & 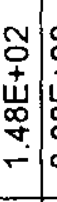 & 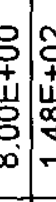 & 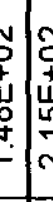 & 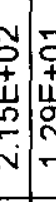 & 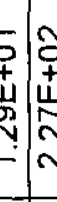 & 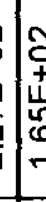 & : & 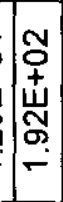 & 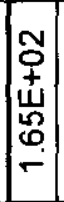 & 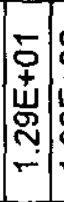 & 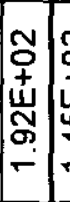 & 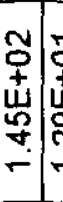 & 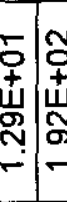 & & : \\
\hline$\frac{5}{5}$ & - & 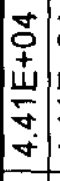 & 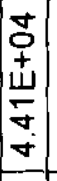 & & \begin{tabular}{|c|c}
$m$ \\
0 \\
4 \\
$w$ \\
$\infty$ \\
$\infty$ \\
$m$
\end{tabular} & \begin{tabular}{|c|}
$m$ \\
0 \\
+ \\
$w$ \\
$\infty$ \\
$\infty$ \\
$m$
\end{tabular} & & 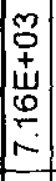 & $\begin{array}{c}3 \\
0 \\
+ \\
0 \\
0 \\
\\
\end{array}$ & & $\begin{array}{c}m \\
0 \\
+ \\
w \\
m \\
0 \\
0 \\
0\end{array}$ & $\begin{array}{l}m \\
0 \\
+ \\
w \\
0 \\
0 \\
\omega \\
0\end{array}$ & & 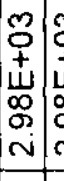 & $\begin{array}{c}3 \\
0 \\
+ \\
w \\
o \\
\vdots \\
i \\
\end{array}$ & & 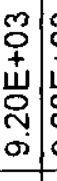 & $\begin{array}{c}1 \\
1 \\
3 \\
0 \\
0 \\
\end{array}$ & & 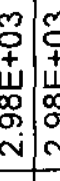 & & & 隽 & & 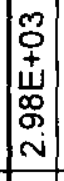 & 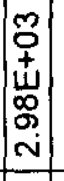 & & 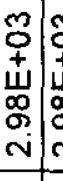 & 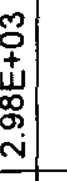 & & 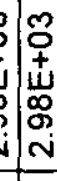 \\
\hline 通 & & $\left|\begin{array}{c}n \\
0 \\
+ \\
m \\
m \\
\\
\end{array}\right|$ & $\left|\begin{array}{c}n \\
0 \\
+ \\
w \\
m \\
\cdots \\
ن \\
v\end{array}\right|$ & & $\left|\begin{array}{c}0 \\
0 \\
+ \\
w \\
\\
\sim \\
v\end{array}\right|$ & $\begin{array}{l}n \\
0 \\
+ \\
\\
\\
\end{array}$ & & $\left|\begin{array}{c}n \\
0 \\
\vdots \\
w \\
\infty \\
\infty \\
- \\
-\end{array}\right|$ & $\left|\begin{array}{c}5 \\
0 \\
+ \\
w \\
\infty \\
\infty \\
-\end{array}\right|$ & & $\mid \begin{array}{c}n \\
0 \\
+ \\
w \\
n \\
\infty \\
- \\
-\end{array}$ & $\begin{array}{l}n \\
0 \\
+ \\
4 \\
0 \\
0 \\
0 \\
-\end{array}$ & & 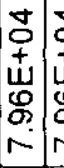 & 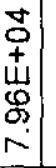 & & 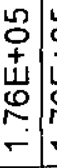 & $\begin{array}{l}0 \\
\vdots \\
1 \\
0 \\
0 \\
-\end{array}$ & & 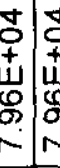 & & & 8 & & 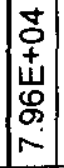 & 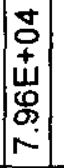 & & 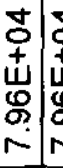 & & & 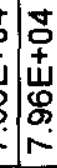 \\
\hline o & $\left(\begin{array}{l}y \\
-3\end{array}\right.$ & $\left|\begin{array}{c} \pm \\
0 \\
⿱ 亠 䒑 \\
\infty \\
\sim \\
0\end{array}\right|$ & $\left|\begin{array}{c}+ \\
0 \\
+ \\
w \\
\infty \\
\sim \\
\sigma\end{array}\right|$ & & \begin{tabular}{|l}
$n$ \\
0 \\
+ \\
$w$ \\
$=$ \\
\\
\end{tabular} & 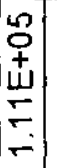 & & $\mid \begin{array}{c}n \\
0 \\
+ \\
w \\
0 \\
- \\
-\end{array}$ & $\left|\begin{array}{c}n \\
0 \\
+ \\
w \\
0 \\
m \\
-\end{array}\right|$ & & $\begin{array}{c}\infty \\
0 \\
+ \\
\infty \\
\sim \\
-1\end{array}$ & 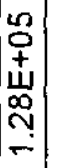 & & 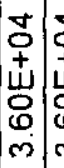 & $\begin{array}{l}0 \\
0 \\
+ \\
\vdots \\
0 \\
0 \\
\dot{m}\end{array}$ & & 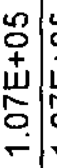 & 足 & & 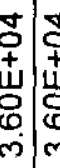 & & & 8 & & 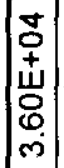 & $\left|\begin{array}{c}0 \\
0 \\
+ \\
\dot{0} \\
0 \\
m \\
m\end{array}\right|$ & & 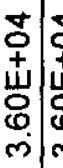 & $\begin{array}{l} \\
0 \\
+ \\
\\
0 \\
\vdots \\
\vdots\end{array}$ & & 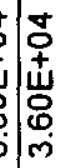 \\
\hline$\frac{x}{6}$ & 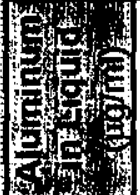 & 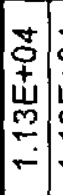 & $\left|\begin{array}{c}0 \\
0 \\
+ \\
m \\
0\end{array}\right|$ & & 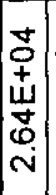 & 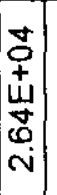 & & 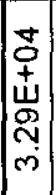 & $\begin{array}{c} \pm \\
0 \\
+ \\
w \\
0 \\
\sim \\
m \\
m\end{array}$ & & 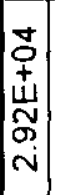 & 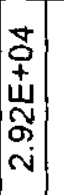 & & 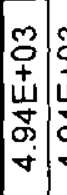 & $\begin{array}{l}m \\
0 \\
+ \\
w \\
0 \\
\dot{v} \\
\dot{v}\end{array}$ & & 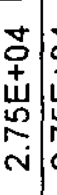 & & & 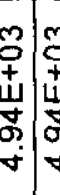 & & & 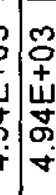 & & 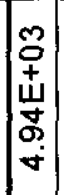 & 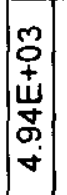 & & 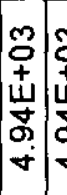 & & & 18 \\
\hline 然 & W & 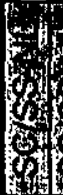 & 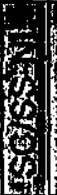 & 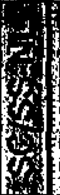 & 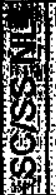 & 列 & & 6 & 6 & 3 & 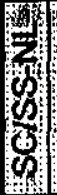 & $\frac{1}{6 !}$ & 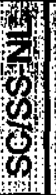 & mat & 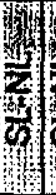 & 整新 & 8 & 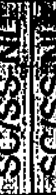 & & 4 & $y^{4}$ & & 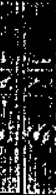 & & $f$ & & & & & & \\
\hline 15 & $5^{2} x_{4}, 4$ & $1-$ & -1 & $\infty$ & 1 & $\Delta$ & $\infty$ & 1 & 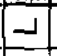 & 0 & $1-$ & $\triangle \mathrm{JC}$ & 0 & $1-$ & $\triangle 1$ & 01 & 1 & $\theta 0$ & & $1=$ & 10 & 1 & -1 & 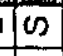 & $1-$ & -1 & $\omega \mathrm{r}$ & -1 & -10 & 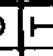 & لـ \\
\hline$\frac{6}{6}$ & 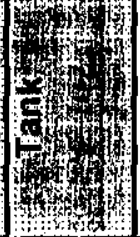 & $\mid$ & $\left|\begin{array}{l}.9 \\
\frac{9}{2} \\
\frac{9}{9} \\
0 \\
7 \\
\\
\end{array}\right|$ & $\mid \begin{array}{l}0 \\
0 \\
0 \\
0 \\
0 \\
\\
\supset\end{array}$ & $\mid$ & 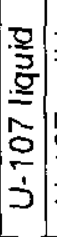 & in & 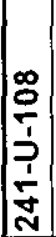 & 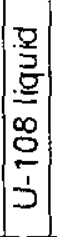 & $\mid \begin{array}{l}0 \\
0 \\
0 \\
\infty \\
0 \\
0\end{array}$ & $\mid$ & $\begin{array}{l}\frac{g}{3} \\
\frac{a}{5} \\
\frac{2}{0} \\
\frac{6}{3}\end{array}$ & 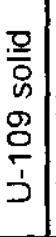 & $\mid$ & $\begin{array}{l}\text { 윽 } \\
: \underline{5} \\
0 \\
\frac{9}{7} \\
\end{array}$ & $\begin{array}{l}\frac{9}{5} \\
0 \\
0 \\
0 \\
\\
j\end{array}$ & 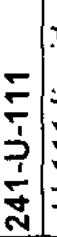 & $\frac{5}{\frac{9}{5}}$ & (s) & $\frac{7}{2}$ & 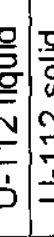 & $\underbrace{n}_{5}$ & 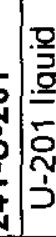 & 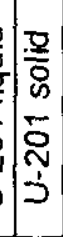 & 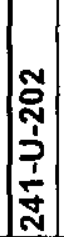 & 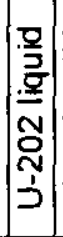 & 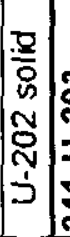 & $\mid$ & 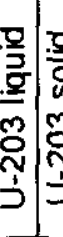 & 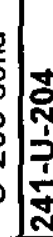 & 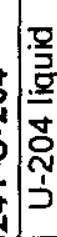 \\
\hline
\end{tabular}




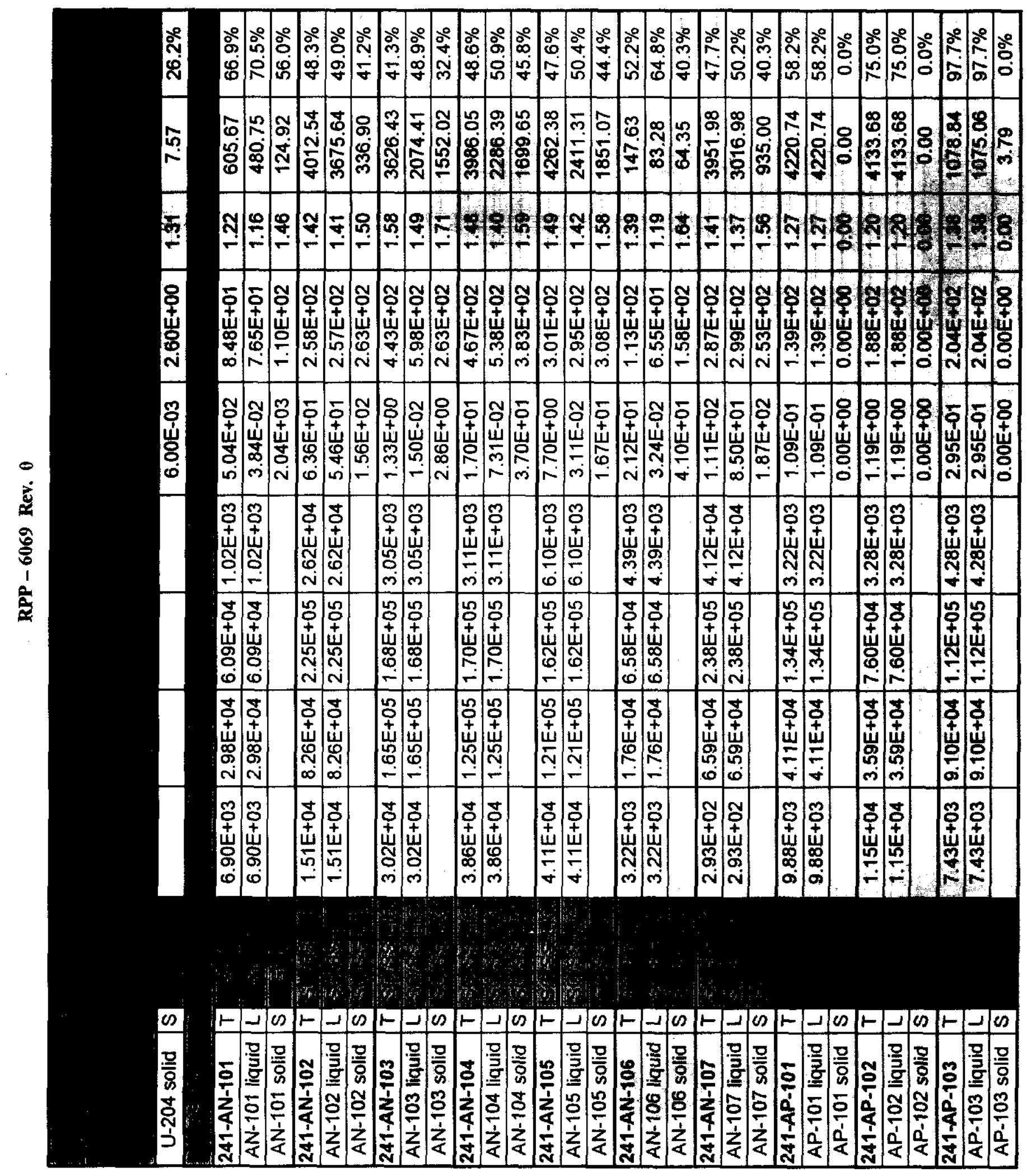




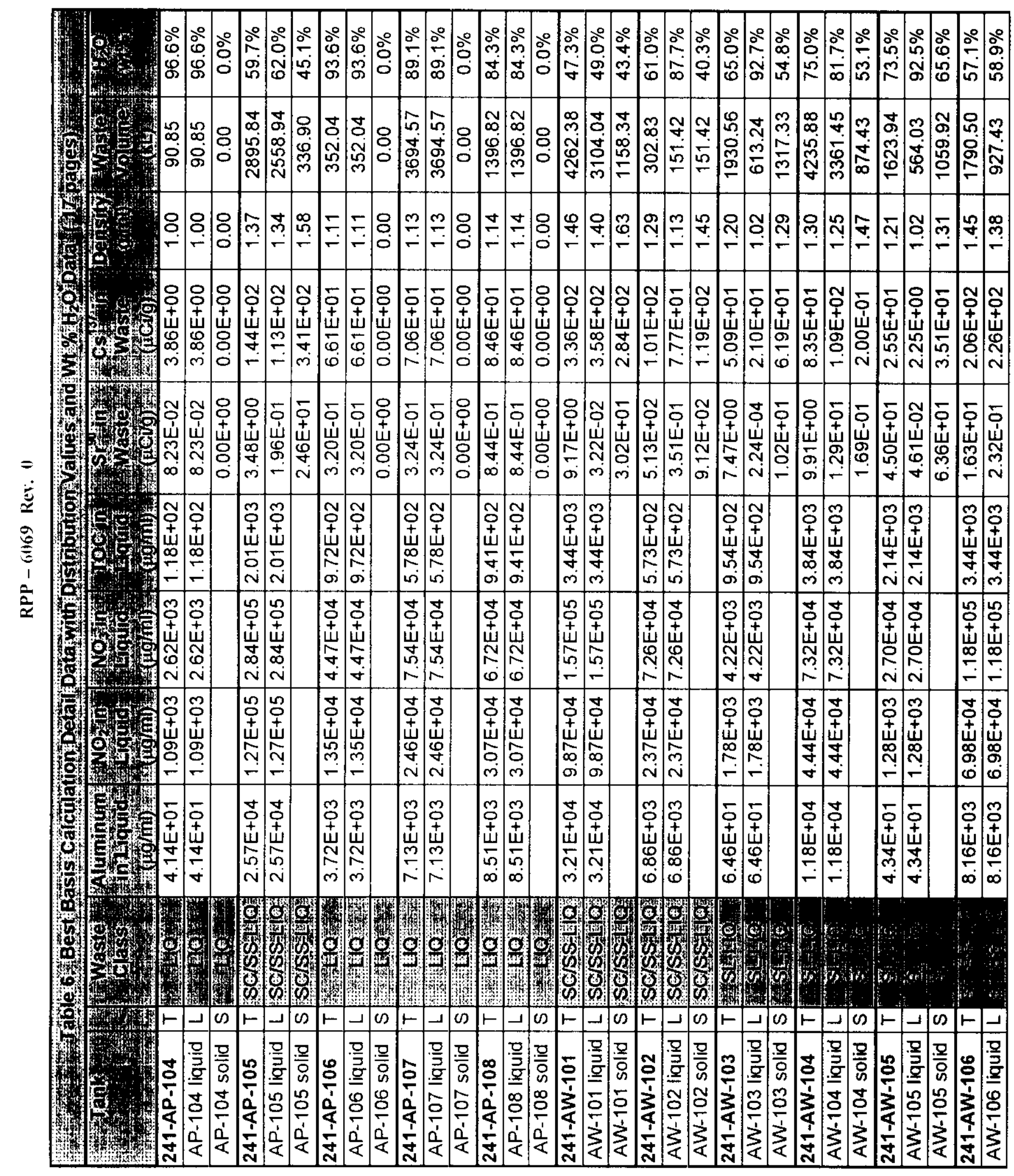




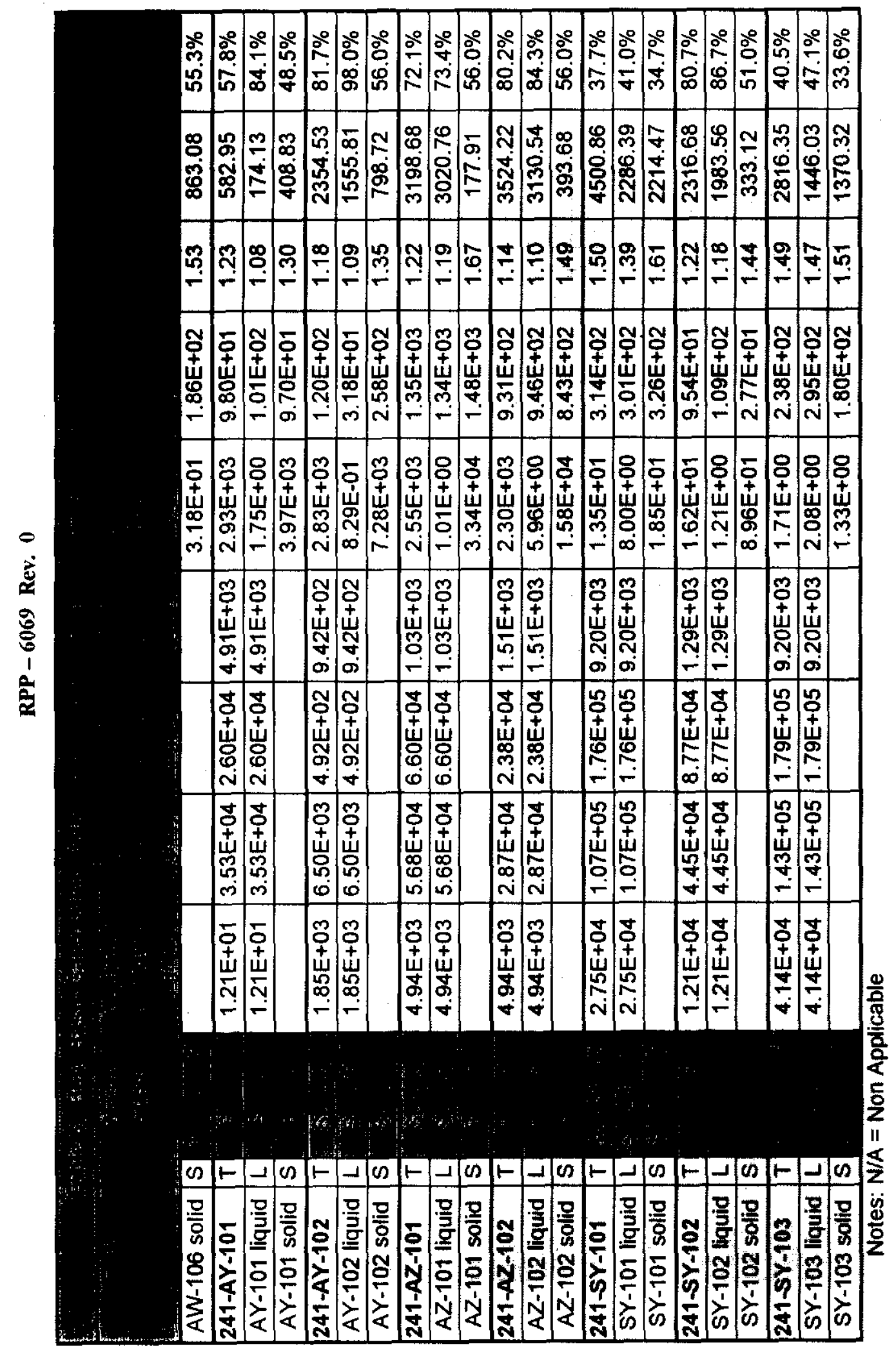


RPP - 60669 Rev. ()

This page intentionally left blank 


\subsection{Results}

The source data of the constituents $\left(\mathrm{Al}, \mathrm{NO}_{2}, \mathrm{NO}_{3}, \mathrm{TOC}, \mathrm{Sr}^{90}\right.$ and $\left.\mathrm{Cs}^{137}\right)$ for this report were gathered from the Best Basis Inventory Management system and is contained in Table 2 with distribution numbers inserted for the missing data values. The weight percent $\mathrm{H}_{2} \mathrm{O}$ data were collected on the TCD database and is recorded in Table 3, also with distribution numbers for the missing values. The mean values of the distributions, from Table 5, were input for the missing values and then documented in Table 6.

Table 6 reflects the best available data values for the desired parameters in the Single Shell (SS) and Double Shell (DS) waste tanks, taken from the Best Basis Calculation Detail. With the exception of the $\mathrm{H}_{2} \mathrm{O}$ weight percent values which were taken from the Tank Characterization Database. The data hierarchy used for this report was BBI values first, distribution values next and if needed TCD values. For the weight percent $\mathrm{H}_{2} \mathrm{O}$ the hierarchy was TCD values first, BBI values next and lastly distributions.

Waste tank 241-A-105 had no associated wt $\% \mathrm{H}_{2} \mathrm{O}$ values in the TCD and the calculated distribution values were not consistent with process knowledge of tank attributes. There is no appreciable liquid layer in this waste tank, due to the high temperatures and length of time since there were any water additions to the waste. Therefore the wt $\% \mathrm{H}_{2} \mathrm{O}$ in the tank was assessed a value of N/A (Non Applicable) for all layers.

\subsection{References}

Barker, S. A., 2000, "Determination of Tank Waste Groupings and Volumes", RPP-6171, Rev. 0, CH2M HILL Hanford Group, Inc, Richland, WA.

LMHC, 1999, Data Source Selection/Best Basis Inventory/ Best Basis Calculation Detail, Tank Characterization Database, Internet at http://twins.pnl.gov/data/getLookupFields3.exe?table=autotcr.dbo.v_calc_detail.

LMHC, 1999, Data Source Selection/ Sample Analysis/ Tank Results RPP 241 (show QA), Tank Characterization Database, Internet at http://twins.pnl.gov/data/getLookupFields3.exe?table=tcd.dbo.p_tank_results_241_show_qa 
RPP - 6069 Rev. (1

This page intentionally left blank 


\section{CHECKLIST FOR INDEPENDENT REVIEW}

Document:

Author:

Scope of Review:
Hydrogen Generation Rate Model Calculation Input Data M.A. Ku fahl and D.C. Hedengren Reviewed complete document. Approtimately 1 \%o of input and output chectled.

Previous reviews complete and cover analysis, up to scope of the review, with no gaps.

Q [ ] [ ] Problem completely defined.

$\infty[]$ [ $]$

[ ] [ ]

D.

Necessary assumptions explicitly stated and supported.

[]$\bowtie$

[]

Computer codes and data files documented.

$W^{+}[$

Data used in calculations explicitly stated in document.

Data checked for consistency with original source information as applicable. + Approximately 1\%, of in put data chectfed

$\bowtie \quad[] \quad[]$

Mathematical derivations checked including dimensional consistency of results.

$\mathrm{X}^{++}[$

Models appropriate and used within range of validity or use outside range of established validity justified.

Hand calculations checked for errors. Spreadsheet results should be treated excictly the same as hand calculations. +t Approximately Software/Code input correct and consistent with analysis documentation.

] Software/Code output consistent with input and with results reported in analysis/documentation. t+t Approximately 1\%o cheotted Limits/criteria/guidelines applied to analysis results are appropriate and referenced. Limits/criteria/guidelines checked against references.

$\begin{array}{lll}{[]} & {[]} & \bigotimes \\ {[]} & {[]} & \bigotimes \\ X] & {[]} & {[]}\end{array}$ Safety margins consistent with good engineering practices.

Conclusions consistent with analytical results and applicable limits.

Results and conclusions address all points required in the problem statement.

X] [ ] [ ] Format consistent with appropriate standards.

[ ] [ ] $\bigotimes^{*}$ Review calculations, comments, and/or notes attached.

¿ [ ] [ ] Document approved.

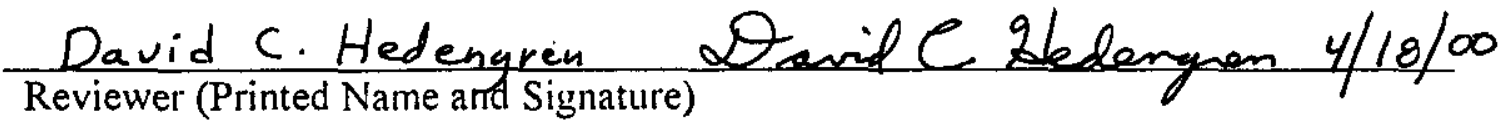

* Any calculation, comments or notes generated as part of this review should be signed, dated, and attached to the checklist. Such material should be labeled and recorded in such a manner as to be intelligible to a technically qualitied third party. 